TRANSACTIONS OF THE

AMERICAN MATHEMATICAL SOCIETY

Volume 352, Number 8, Pages 3837-3879

S 0002-9947(00)02394-1

Article electronically published on March 24, 2000

\title{
EXTENSIONS OF HOPF ALGEBRAS AND LIE BIALGEBRAS
}

\author{
AKIRA MASUOKA
}

Dedicated to Professor Bodo Pareigis on his sixtieth birthday

\begin{abstract}
Let $\mathfrak{f}, \mathfrak{g}$ be finite-dimensional Lie algebras over a field of characteristic zero. Regard $\mathfrak{f}$ and $\mathfrak{g}^{*}$, the dual Lie coalgebra of $\mathfrak{g}$, as Lie bialgebras with zero cobracket and zero bracket, respectively. Suppose that a matched pair $\left(\mathfrak{f}, \mathfrak{g}^{*}\right)$ of Lie bialgebras is given, which has structure maps $\rightarrow, \rho$. Then it induces a matched pair $\left(U \mathfrak{f}, U \mathfrak{g}^{\circ}, \rightarrow^{\prime}, \rho^{\prime}\right)$ of Hopf algebras, where $U \mathfrak{f}$ is the universal envelope of $\mathfrak{f}$ and $U \mathfrak{g}^{\circ}$ is the Hopf dual of $U \mathfrak{g}$. We show that the group $\operatorname{Opext}\left(U \mathfrak{f}, U \mathfrak{g}^{\circ}\right)$ of cleft Hopf algebra extensions associated with $\left(U \mathfrak{f}, U \mathfrak{g}^{\circ}, \rightarrow^{\prime}, \rho^{\prime}\right)$ is naturally isomorphic to the group Opext $\left(\mathfrak{f}, \mathfrak{g}^{*}\right)$ of Lie bialgebra extensions associated with $\left(\mathfrak{f}, \mathfrak{g}^{*}, \rightarrow, \rho\right)$. An exact sequence involving either of these groups is obtained, which is a variation of the exact sequence due to G.I. Kac. If $\mathfrak{g}=[\mathfrak{g}, \mathfrak{g}]$, there follows a bijection between the set $\operatorname{Ext}\left(U \mathfrak{f}, U \mathfrak{g}^{\circ}\right)$ of all cleft Hopf algebra extensions of $U \mathfrak{f}$ by $U \mathfrak{g}^{\circ}$ and the set $\operatorname{Ext}\left(\mathfrak{f}, \mathfrak{g}^{*}\right)$ of all Lie bialgebra extensions of $\mathfrak{f}$ by $\mathfrak{g}^{*}$.
\end{abstract}

\section{INTRODUCTION}

Let us recall the theory of group extensions with abelian kernels. Let $\Gamma$ be a group and let $M$ be a left $\Gamma$-module with structure $\rightarrow: \Gamma \times M \rightarrow M$. The equivalence classes of the group extensions of $\Gamma$ by $M$ giving rise to $\rightarrow$ form an abelian group Opext $(\Gamma, M)$ under the Baer product, which is isomprphic to the 2nd cohomology group $H^{2}(\Gamma, M)$ of $\Gamma$ with coefficients $M$.

The theory was extended to the theory of Hopf algebra extensions which are abelian in some sense (due to Singer [S] in the graded case, and Hofstetter [Hf] in the ungraded case). We work over a ground field $k$. Let $(H, K, \rightarrow, \rho)$ be an (abelian) matched pair of Hopf algebras, for which we will propose the term 'Singer pair' in the text. This means that $H, K$ are Hopf algebras, where $H$ is cocommutative and $K$ is commutative, and that $\rightarrow: H \otimes K \rightarrow K$ and $\rho: H \rightarrow H \otimes K$ are an action and a coaction satisfying certain compatibility conditions. The equivalence classes of the cleft (Hopf algebra) extensions $(A)=K \rightarrow A \rightarrow H$ of $H$ by $K$ giving rise to $\rightarrow$ and $\rho$ form an abelian group $\operatorname{Opext}(H, K)$, which permits a cohomological description. Here a Hopf algebra extension $(A)$ is said to be cleft, if there is a

Received by the editors May 23, 1997 and, in revised form, April 10, 1998.

2000 Mathematics Subject Classification. Primary 16W30; Secondary 17B37, 17B56.

Key words and phrases. Extension, Hopf algebra, Lie bialgebra, Lie algebra cohomology, continuous modules.

This work was done at the Forschungsstipendiat der Alexander von Humboldt-Stiftung. The revision was done during a visit to the FaMAF, University of Córdoba. Their hospitality is gratefully acknowledged. 
left $K$-linear and right $H$-colinear isomorphism $A \cong K \otimes H$. If $H, K$ are both finite-dimensional, all extensions are necessarily cleft.

Prior to $[\underline{\mathrm{S}}$ and $[\mathrm{Hf}]$, Kac [ $\mathrm{Kac}]$ had established the theory in the special case where $H=k F$, a group algebra, and $K=k^{G}$, the dual of such an algebra, with $F, G$ finite groups. (Precisely, he worked on Hopf algebras with $*$-structure over the complex numbers $\mathbb{C}$, which are nowadays called Kac algebras.) It should be remarked that he showed an interesting exact sequence involving $\operatorname{Opext}\left(k F, k^{G}\right)$, which has been so far a peculiar result when $(H, K)=\left(k F, k^{G}\right)$.

Let $\mathfrak{f}, \mathfrak{g}$ be finite-dimensional Lie algebras. The universal enveloping algebra $U \mathfrak{f}$ of $\mathfrak{f}$ is naturally a cocommutative Hopf algebra, and hence the dual Hopf algebra $U \mathfrak{g}^{\circ}$ of $U \mathfrak{g}$ is a commutative Hopf algebra. In this paper, we shall discuss relations of cleft extensions of $U \mathfrak{f}$ by $U \mathfrak{g}^{\circ}$ and Lie bialgebra extensions of $\mathfrak{f}$ by $\mathfrak{g}^{*}$, and thereby obtain a variation of the Kac exact sequence for $\left(U \mathfrak{f}, U \mathfrak{g}^{\circ}\right)$.

A Lie coalgebra is the dual object of a Lie algebra, and its structure is called a cobracket. A Lie bialgebra is a Lie algebra and Lie coalgebra which satisfies some compatibility condition. We regard $\mathfrak{f}$ as a Lie bialgebra with zero cobracket. The linear dual $\mathfrak{g}^{*}$ of $\mathfrak{g}$ is a Lie coalgebra, which is regarded as a Lie bialgebra with zero bracket. The notion of a matched pair $\left(\mathfrak{f}, \mathfrak{g}^{*}, \rightarrow, \rho\right)$ is defined by Majid $[\mathrm{Mj}]$; it also will be called a 'Singer pair' in the text. Fix such a pair $\left(\mathfrak{f}, \mathfrak{g}^{*}\right)$ (here and in the following, $(\rightarrow, \rho)$, viewed as structure, is omitted). We define a set, in fact an abelian group, Opext $\left(\mathfrak{f}, \mathfrak{g}^{*}\right)$ which consists of the equivalence classes of the Lie bialgebra extensions of $\mathfrak{f}$ by $\mathfrak{g}^{*}$ giving rise to $\rightarrow, \rho$, and show an exact sequence involving it, which is a Lie bialgebra version of the Kac exact sequence (see Theorem $2.10)$.

It is seen that the matched pair $\left(\mathfrak{f}, \mathfrak{g}^{*}\right)$ of Lie bialgebras induces in a natural way a matched pair $\left(U \mathfrak{f}, U \mathfrak{g}^{\circ}\right)$ of Hopf algebras. In the following, suppose that the characteristic ch $k$ of $k$ is zero. We prove that there is a natural isomorphism of groups

$$
\operatorname{Opext}\left(U \mathfrak{f}, U \mathfrak{g}^{\circ}\right) \cong \operatorname{Opext}\left(\mathfrak{f}, \mathfrak{g}^{*}\right) .
$$

The unit of these groups is represented by the split extensions $\left(U \mathfrak{g}^{\circ} \# U \mathfrak{f}\right),\left(\mathfrak{g}^{*} \bowtie \mathfrak{f}\right)$, respectively. It is shown that the (abelian) groups $\operatorname{Aut}\left(U \mathfrak{g}^{\circ} \# U \mathfrak{f}\right)$, Aut $\left(\mathfrak{g}^{*} \bowtie \mathfrak{f}\right)$ of the auto-equivalences of the split extensions are isomorphic:

$$
\operatorname{Aut}\left(U \mathfrak{g}^{\circ} \# U \mathfrak{f}\right) \cong \operatorname{Aut}\left(\mathfrak{g}^{*} \bowtie \mathfrak{f}\right) .
$$

These isomorphisms make it easier to compute the groups in the left-hand side, since those in the right-hand side are much easier to compute (see Corollary 4.13, Example 4.19). Furthermore, they produce, combined with the Lie bialgebra version of the Kac exact sequence, another variation:

$$
\begin{aligned}
0 & \rightarrow H^{1}(\mathfrak{f} \bowtie \mathfrak{g}, k) \rightarrow H^{1}(\mathfrak{f}, k) \oplus H^{1}(\mathfrak{g}, k) \rightarrow \operatorname{Aut}\left(U \mathfrak{g}^{\circ} \# U \mathfrak{f}\right) \\
& \rightarrow H^{2}(\mathfrak{f} \bowtie \mathfrak{g}, k) \rightarrow H^{2}(\mathfrak{f}, k) \oplus H^{2}(\mathfrak{g}, k) \rightarrow \operatorname{Opext}\left(U \mathfrak{f}, U \mathfrak{g}^{\circ}\right) \\
& \rightarrow H^{3}(\mathfrak{f} \bowtie \mathfrak{g}, k) \rightarrow H^{3}(\mathfrak{f}, k) \oplus H^{3}(\mathfrak{g}, k) \quad(\text { exact }),
\end{aligned}
$$

where $\mathfrak{f} \bowtie \mathfrak{g}$ is a certain Lie algebra constructed from the matched pair $\left(\mathfrak{f}, \mathfrak{g}^{*}\right)$ and $H$ indicates the cohomology group of Lie algebras with coefficients the trivial Lie module $k$.

Suppose that $\mathfrak{g}=[\mathfrak{g}, \mathfrak{g}]$ (this holds if $\mathfrak{g}$ is semisimple). Then, since we see that every matched pair $\left(U \mathfrak{f}, U \mathfrak{g}^{\circ}\right)$ of Hopf algebras is induced from a uniquely 
determined matched pair $\left(\mathfrak{f}, \mathfrak{g}^{*}\right)$ of Lie bialgebras, there follows a bijection

$$
\operatorname{Ext}\left(U \mathfrak{f}, U \mathfrak{g}^{\circ}\right) \cong \operatorname{Ext}\left(\mathfrak{f}, \mathfrak{g}^{*}\right)
$$

between the set $\operatorname{Ext}\left(U \mathfrak{f}, U \mathfrak{g}^{\circ}\right)$ of the equivalence classes of all cleft etensions of $U \mathfrak{f}$ by $U \mathfrak{g}^{\circ}$ and the set $\operatorname{Ext}\left(\mathfrak{f}, \mathfrak{g}^{*}\right)$ of the equivalence classes of all Lie bialgebra extensions of $\mathfrak{f}$ by $\mathfrak{g}^{*}$.

The idea of the proof for the isomorphisms (0.1), (0.2) comes from the familiar uniqueness of injective resolutions. In fact, we see that the groups in the left-hand side and in the right-hand side are derived from two resolutions of the same left module over $U=U(\mathfrak{f} \bowtie \mathfrak{g})$, for which a theorem due to Schneider (Theorem 5.2) on vanishing of Lie algebra cohomology is crucial. It is a key fact that low terms in these resolutions look like injective objects in some non-abelian category which consists of left $U$-modules with topology (see Section 6).

Notation. Throughout, $k$ denotes the ground field whose characteristic $\operatorname{ch} k$ is arbitrary, but is often assumed to be zero. Vector spaces and linear maps are over $k$, and the tensor products $\otimes$ and the exterior products $\wedge$ are taken over $k$, unless otherwise stated. Let $V, W$ be vector spaces. Let $\operatorname{Hom}(V, W)$ denote the vector space of the linear maps $V \rightarrow W$, and let $V^{*}$ denote the dual vector space $\operatorname{Hom}(V, k)$ of $V$. The value $f(v)$ of $f \in V^{*}$ at $v \in V$ is also denoted by $\langle f, v\rangle$ or by $\langle v, f\rangle$. The identity map of $V$ is denoted by $1: V \rightarrow V$.

\section{Lie Bialgebra Extensions}

Let $\mathfrak{f}$ and $\mathfrak{g}$ be finite-dimensional Lie algebras.

Definition 1.1 Mj. Definition 8.3.1]. A pair $(\mathfrak{f}, \mathfrak{g})$ equipped with actions

$$
\triangleright: \mathfrak{g} \otimes \mathfrak{f} \rightarrow \mathfrak{f}, \quad \triangleleft: \mathfrak{g} \otimes \mathfrak{f} \rightarrow \mathfrak{g}
$$

is called a matched pair of Lie algebras, if $\mathfrak{f}$ is a left $\mathfrak{g}$-Lie module under $\triangleright$, if $\mathfrak{g}$ is a right $\mathfrak{f}$-Lie module under $\triangleleft$, and if

$$
\begin{aligned}
& \text { 1) } x \triangleright[a, b]=[x \triangleright a, b]+[a, x \triangleright b]+(x \triangleleft a) \triangleright b-(x \triangleleft b) \triangleright a, \\
& \text { 2) }[x, y] \triangleleft a=[x, y \triangleleft a]+[x \triangleleft a, y]+x \triangleleft(y \triangleright a)-y \triangleleft(x \triangleright a)
\end{aligned}
$$

for $a, b \in \mathfrak{f}, x, y \in \mathfrak{g}$.

These conditions are equivalent to the condition that the direct sum $\mathfrak{f} \oplus \mathfrak{g}$ of the vector spaces forms a Lie algebra under the bracket defined by

$$
[a \oplus x, b \oplus y]=([a, b]+x \triangleright b-y \triangleright a) \oplus([x, y]+x \triangleleft b-y \triangleleft a),
$$

where $a, b \in \mathfrak{f}, x, y \in \mathfrak{g}$ (see $\mathrm{Mj}$, Proposition 8.3.2]). This Lie algebra is denoted by $\mathfrak{f} \bowtie \mathfrak{g}$.

Let $\mathfrak{h}$ be a Lie algebra including $\mathfrak{f}, \mathfrak{g}$ as Lie subalgebras so that $\mathfrak{h}=\mathfrak{f} \oplus \mathfrak{g}$ as a vector space. It is shown in $\mathrm{Mj}$ part of Proposition 8.3.2] that the actions determined by

$$
[x, a]=x \triangleright a \oplus x \triangleleft a \quad(x \in \mathfrak{g}, a \in \mathfrak{f})
$$

make $(\mathfrak{f}, \mathfrak{g})$ into a matched pair, and then $\mathfrak{h}=\mathfrak{f} \bowtie \mathfrak{g}$ as a Lie algebra. Conversely, every matched pair $(\mathfrak{f}, \mathfrak{g}, \triangleright, \triangleleft)$ is obtained in this way.

Example 1.2. Let $\mathfrak{f}=k a, \mathfrak{g}=k x$ be 1-dimensional (necessarily abelian) Lie algebras. For arbitrary $s, t \in k$, the actions defined by

$$
x \triangleright a=s a, \quad x \triangleleft a=t x
$$


makes the pair $(\mathfrak{f}, \mathfrak{g})$ matched, since the bracket determined by

$$
[x, a]=s a \oplus t x
$$

defines a Lie algebra structure on the 2-dimensional vector space $\mathfrak{h}=k a \oplus k x$.

A Lie coalgebra Mi is a vector space $\mathfrak{l}$ equipped with a linear map $\delta: \mathfrak{l} \rightarrow \mathfrak{l} \otimes \mathfrak{l}$, called a cobracket, satisfying the coanticommutativity and the co-Jacobi identity, that is,

$$
(1-\tau) \delta=0 \quad \text { and } \quad\left(1+\xi+\xi^{2}\right)(1 \otimes \delta) \delta=0,
$$

where $\tau: \mathfrak{l} \otimes \mathfrak{l} \rightarrow \mathfrak{l} \otimes \mathfrak{l}, \xi: \mathfrak{l} \otimes \mathfrak{l} \otimes \mathfrak{l} \rightarrow \mathfrak{l} \otimes \mathfrak{l} \otimes \mathfrak{l}$ are the twistings defined by

$$
\tau(u \otimes v)=v \otimes u, \quad \xi(u \otimes v \otimes w)=v \otimes w \otimes u \quad(u, v, w \in \mathfrak{l}) .
$$

A Lie bialgebra $[\mathrm{D}$ is a Lie algebra and Lie coalgebra $\mathfrak{l}$ satisfying

$$
\begin{aligned}
\delta[u, v] & =\sum\left[u, v_{[1]}\right] \otimes v_{[2]}+\sum v_{[1]} \otimes\left[u, v_{[2]}\right] \\
& +\sum\left[u_{[1]}, v\right] \otimes u_{[2]}+\sum u_{[1]} \otimes\left[u_{[2]}, v\right]
\end{aligned}
$$

for $u, v \in \mathfrak{l}$, where $\delta u=\sum u_{[1]} \otimes u_{[2]}$.

Any Lie algebra (respectively, Lie coalgebra) is a Lie bialgebra with zero cobracket (respectively, zero bracket). We regard $\mathfrak{f}$ as a Lie bialgebra with zero cobracket. The linear dual $\mathfrak{g}^{*}$ of $\mathfrak{g}$ is a Lie coalgebra whose cobracket $\delta$ is the dual map of the bracket of $\mathfrak{g}$. We regard $\mathfrak{g}^{*}$ as a Lie bialgebra with zero bracket.

A right $\mathfrak{g}^{*}$-Lie comodule structure [Mj, p. 382] on $\mathfrak{f}$ is a linear map $\rho: \mathfrak{f} \rightarrow \mathfrak{f} \otimes \mathfrak{g}^{*}$ satisfying $(1 \otimes \delta) \rho=(1 \otimes(1-\tau))(\rho \otimes 1) \rho$.

Definition 1.5. A pair $\left(\mathfrak{f}, \mathfrak{g}^{*}\right)$ equipped with an action and a coaction,

$$
\rightarrow: \mathfrak{f} \otimes \mathfrak{g}^{*} \rightarrow \mathfrak{g}^{*} \quad \text { and } \quad \rho: \mathfrak{f} \rightarrow \mathfrak{f} \otimes \mathfrak{g}^{*}, \quad \rho a=\sum a_{[0]} \otimes a_{[1]},
$$

is called a Singer pair of Lie bialgebras, if $\mathfrak{g}^{*}$ is a left $\mathfrak{f}$-Lie module under $\rightarrow$, if $\mathfrak{f}$ is a right $\mathfrak{g}^{*}$-Lie comodule under $\rho$, and if

$$
\begin{aligned}
\delta(a \rightarrow f) & =\sum\left(a \rightarrow f_{[1]}\right) \otimes f_{[2]}+\sum f_{[1]} \otimes\left(a \rightarrow f_{[2]}\right) \\
& +\sum(1-\tau)\left(\left(a_{[0]} \rightarrow f\right) \otimes a_{[1]}\right), \\
\rho[a, b] & =\sum\left[a, b_{[0]}\right] \otimes b_{[1]}+\sum b_{[0]} \otimes\left(a \rightarrow b_{[1]}\right) \\
& -\sum\left[b, a_{[0]}\right] \otimes a_{[1]}-\sum a_{[0]} \otimes\left(b \rightarrow a_{[1]}\right)
\end{aligned}
$$

for $a, b \in \mathfrak{f}, f \in \mathfrak{g}$.

This is defined in [Mj, p. 383] (in a more general situation) and is called a matched pair. However we avoid the term for fear of confusion with the notion in Definition 1.1. See Definition 3.3.

It follows from [Mj, Proposition 8.3.5] that the conditions given above are equivalent to the direct sum $\mathfrak{g}^{*} \oplus \mathfrak{f}$ forming a Lie bialgebra, equipped with the bracket $[,]_{0}$ and the cobracket $\delta_{0}$ defined by

$$
\begin{gathered}
{[f \oplus a, g \oplus b]_{0}=(a \rightarrow g-b \rightarrow f) \oplus[a, b],} \\
\delta_{0}(f \oplus a)=\delta f+(1-\tau) \rho(a)
\end{gathered}
$$

for $f, g \in \mathfrak{g}^{*}, a, b \in \mathfrak{f}$. This Lie bialgebra is denoted by $\mathfrak{g}^{*} \bowtie \mathfrak{f}$. 
Proposition 1.6. There is a natural 1-1 correspondence between the structures $(\triangleright, \triangleleft)$ of a matched pair on $(\mathfrak{f}, \mathfrak{g})$ and the structures $(\rightarrow, \rho)$ of a Singer pair on $\left(\mathfrak{f}, \mathfrak{g}^{*}\right)$.

Proof. The right $\mathfrak{f}$-Lie module structures $\triangleleft: \mathfrak{g} \otimes \mathfrak{f} \rightarrow \mathfrak{g}$ on $\mathfrak{g}$ are in 1-1 correspondence with the left $\mathfrak{f}$-Lie module structures $\rightarrow: \mathfrak{f} \otimes \mathfrak{g}^{*} \rightarrow \mathfrak{g}^{*}$ on $\mathfrak{g}^{*}$, via transpose. The left $\mathfrak{g}$-Lie module structures $\triangleright: \mathfrak{g} \otimes \mathfrak{f} \rightarrow \mathfrak{f}$ on $\mathfrak{f}$ are in 1-1 correspondence with the right $\mathfrak{g}^{*}$-Lie comodule structures $\rho: \mathfrak{f} \rightarrow \mathfrak{f} \otimes \mathfrak{g}^{*}$ on $\mathfrak{f}$, so that

$$
\begin{aligned}
x \triangleright a & =\langle 1 \otimes x, \rho a\rangle \quad(x \in \mathfrak{g}, a \in \mathfrak{f}), \\
\rho a & =\sum_{i}\left(x_{i} \triangleright a\right) \otimes f_{i} \quad(a \in \mathfrak{f}),
\end{aligned}
$$

where $\left\{x_{i}\right\},\left\{f_{i}\right\}$ are bases in $\mathfrak{g}, \mathfrak{g}^{*}$ dual to each other. It is straightforward to see that, if $\triangleleft \leftrightarrow \rightarrow$ and $\triangleright \leftrightarrow \rho$ in these correspondences, then $(\triangleright, \triangleleft)$ is a structure of a matched pair on $(\mathfrak{f}, \mathfrak{g})$ if and only if $(\rightarrow, \rho)$ is a structure of a Singer pair on $\left(\mathfrak{f}, \mathfrak{g}^{*}\right)$.

Definition 1.7. A (Lie bialgebra) extension of $\mathfrak{f}$ by $\mathfrak{g}$ is a sequence $0 \rightarrow \mathfrak{g}^{*} \rightarrow \mathfrak{l} \rightarrow$ $\mathfrak{f} \rightarrow 0$ of Lie bialgebras and Lie bialgebra maps which is a short exact sequence of vector spaces. Two extensions of $\mathfrak{f}$ by $\mathfrak{g}^{*}$ with middle terms $\mathfrak{l}, \mathfrak{l}^{\prime}$ are equivalent, if there is a Lie bialgebra map (necessarily, an isomorphism) $\phi: \mathfrak{l} \rightarrow \mathfrak{l}^{\prime}$ which makes the following diagram commute:

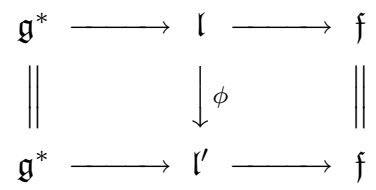

Denote by

\section{$\operatorname{Ext}\left(\mathfrak{f}, \mathfrak{g}^{*}\right)$}

the set of the equivalence classes of all extensions of $\mathfrak{f}$ by $\mathfrak{g}^{*}$.

Fix a left $\mathfrak{f}$-Lie module structure $\rightarrow: \mathfrak{f} \otimes \mathfrak{g}^{*} \rightarrow \mathfrak{g}^{*}$ on $\mathfrak{g}^{*}$, and a right $\mathfrak{g}^{*}$-Lie comodule structure $\rho: \mathfrak{f} \rightarrow \mathfrak{f} \otimes \mathfrak{g}^{*}$ on $\mathfrak{f}$. Let $\triangleleft: \mathfrak{g} \otimes \mathfrak{f} \rightarrow \mathfrak{g}$ be the right $\mathfrak{f}$-Lie module structure corresponding to $\rightarrow$, and let $\triangleright: \mathfrak{g} \otimes \mathfrak{f} \rightarrow \mathfrak{f}$ be the left $\mathfrak{g}$-Lie module structure corresponding to $\rho$ (see the proof of Proposition 1.6). Let

$$
\sigma: \wedge^{2} \mathfrak{f}=\mathfrak{f} \wedge \mathfrak{f} \rightarrow \mathfrak{g}^{*}
$$

be a 2-cocycle for the left $\mathfrak{f}$-Lie module $\left(\mathfrak{g}^{*}, \rightarrow\right)$. In the sequel, we often identify $\sigma$ with the linear map

$$
\mathfrak{g} \otimes \wedge^{2} \mathfrak{f} \rightarrow k, \quad x \otimes(a \wedge b) \mapsto\langle x, \sigma(a, b)\rangle,
$$

which is denoted by $\sigma$, too. We write $\sigma(a, b)$ for $\sigma(a \wedge b)$, and $\sigma(x ; a, b)$ for $\sigma(x \otimes(a \wedge b))$. Let $\leftarrow=\rho^{*}: \mathfrak{f}^{*} \otimes \mathfrak{g} \rightarrow \mathfrak{f}^{*}$ be the right $\mathfrak{g}$-Lie module structure which is the dual map of $\rho$, and let

$$
\theta: \wedge^{2} \mathfrak{g} \rightarrow \mathfrak{f}^{*} \quad \text { or } \quad \theta: \wedge^{2} \mathfrak{g} \otimes \mathfrak{f} \rightarrow k
$$

be a 2-cocycle for $\left(\mathfrak{f}^{*}, \leftarrow\right)$, to which is applied the notational convention similar to $\sigma$. Since $\triangleright$ and $<$ are transposes of each other, the 2-cocycle condition for $\theta$ is given 
by

$$
\begin{aligned}
& \theta(x, y ; z \triangleright a)+\theta(y, z ; x \triangleright a)+\theta(z, x ; y \triangleright a) \\
& \quad=\theta(x,[y, z] ; a)+\theta(y,[z, x] ; a)+\theta(z,[x, y] ; a),
\end{aligned}
$$

where $x, y, z \in \mathfrak{g}, a \in \mathfrak{f}$. Let $[,]_{1}$ be the bracket on the direct sum $\mathfrak{g}^{*} \oplus \mathfrak{f}$ determined by $\rightarrow$ and $\sigma$, or explicitly by

$$
[f \oplus a, g \oplus b]_{1}=(a \rightarrow g-b \rightarrow f+\sigma(a, b)) \oplus[a, b]
$$

for $f, g \in \mathfrak{g}^{*}, a, b \in \mathfrak{f}$. This gives, in fact, a Lie algebra structure (see [We, Exercise 7.7.5]). Define the bracket on $\mathfrak{g} \oplus \mathfrak{f}^{*}$ determined by $\leftarrow$ and $\theta$, and dualize it. Then we have a cobracket, say $\delta_{1}$, on $\mathfrak{g}^{*} \oplus \mathfrak{f}$, with which it is a Lie coalgebra. If $\left\{x_{i}\right\}$, $\left\{f_{i}\right\}$ are bases of $\mathfrak{g}, \mathfrak{g}^{*}$ dual to each other, then one has explicitly

$$
\delta_{1}(f \oplus a)=\delta f+\sum_{i}(1-\tau)\left(\left(x_{i} \triangleright a\right) \otimes f_{i}\right)+\sum_{i, j} \theta\left(x_{i}, x_{j} ; a\right) f_{i} \otimes f_{j}
$$

for $f \in \mathfrak{g}^{*}, a \in \mathfrak{f}$.

Proposition 1.8. $\mathfrak{g}^{*} \oplus \mathfrak{f}$ equipped with $[,]_{1}, \delta_{1}$ is a Lie bialgebra if and only if $(\rightarrow, \rho)$ is a structure of Singer pair on $\left(\mathfrak{f}, \mathfrak{g}^{*}\right)$ and

$$
\begin{aligned}
& \sigma([x, y] ; a, b)+\theta(x, y ;[a, b]) \\
& \quad=\sigma(x ; y \triangleright a, b)+\sigma(x ; a, y \triangleright b)-(x \leftrightarrow y) \\
& \quad+\theta(x, y \triangleleft a ; b)+\theta(x \triangleleft a, y ; b)-(a \leftrightarrow b)
\end{aligned}
$$

for all $x, y \in \mathfrak{g}, a, b \in \mathfrak{f}$. Following $[\mathrm{Mj}]$, the notations $(x \leftrightarrow y),(a \leftrightarrow b)$ are meant to exchange $x$ and $y, a$ and $b$, respectively, in the preceding two terms.

Proof. We will see the necessary and sufficient condition for $\mathfrak{l}=\mathfrak{g}^{*} \oplus \mathfrak{f}$ to satisfy the equation (1.4) in each case where $(u, v)=(f, g),(a, f)$ or $(a, b)$ with $a, b \in \mathfrak{f}, f, g \in$ $\mathfrak{g}^{*}$. By the anticommutativity of $[,]_{1}$, we may omit the case where $(u, v)=(f, a)$.

Suppose $(u, v)=(f, g)$. Then the equation (1.4) holds trivially with both sides zeros.

Suppose $(u, v)=(a, f)$. Then equation (1.4) becomes

$$
\begin{aligned}
\delta(a \rightarrow f)= & \sum\left(a \rightarrow f_{[1]}\right) \otimes f_{[2]}+\sum f_{[1]} \otimes\left(a \rightarrow f_{[2]}\right) \\
& +\sum_{i}(1-\tau)\left\{\left(\left(x_{i} \triangleright a\right) \rightarrow f\right) \otimes f_{i}\right\} .
\end{aligned}
$$

Notice that each side is in $\mathfrak{g}^{*} \otimes \mathfrak{g}^{*}$. Evaluting at $x \otimes y \in \mathfrak{g} \otimes \mathfrak{g}$, we see that (1.4) holds if and only if (1.1.2) holds.

Suppose $(u, v)=(a, b)$. The left-hand side of (1.4) becomes

$$
\begin{gathered}
\sum_{i} \sigma\left(x_{i} ; a, b\right) \delta\left(f_{i}\right)+\sum_{i}(1-\tau)\left\{\left(x_{i} \triangleright[a, b]\right) \otimes f_{i}\right\} \\
+\sum_{i, j} \theta\left(x_{i}, x_{j} ;[a, b]\right) f_{i} \otimes f_{j},
\end{gathered}
$$

while the right-hand side becomes

$$
\begin{aligned}
& \sum_{i}(1-\tau)\left\{\left[a, x_{i} \triangleright b\right] \otimes f_{i}+\sigma\left(a, x_{i} \triangleright b\right) \otimes f_{i}+\left(x_{i} \triangleright b\right) \otimes\left(a \rightarrow f_{i}\right)\right\} \\
& +\sum_{i, j} \theta\left(x_{i}, x_{j} ; b\right)\left\{\left(a \rightarrow f_{i}\right) \otimes f_{j}+f_{i} \otimes\left(a \rightarrow f_{j}\right)\right\}-(a \leftrightarrow b) .
\end{aligned}
$$


Each side vanishes on $\mathfrak{f}^{*} \otimes \mathfrak{f}^{*}$. Evalute each side at $x \otimes y \in \mathfrak{g} \otimes \mathfrak{g}$. Then the equation (1.4) yields the equation in the proposition. Evalute each side at $p \otimes x \in \mathfrak{f}^{*} \otimes \mathfrak{g}$ or at $x \otimes p \in \mathfrak{g} \otimes \mathfrak{f}^{*}$. Then the equation in the proposition yields (1.1.1).

We see now from Proposition 1.6 that the proposition follows.

Suppose that $\rightarrow, \rho, \sigma, \theta$ satisfy the conditions given in the preceding proposition. We denote by $\mathfrak{g}^{*} \triangleleft_{\sigma, \theta} \mathfrak{f}$ the Lie bialgebra equipped with $[,]_{1}, \delta_{1}$, keeping $\rightarrow, \rho$ in mind. If $\sigma, \theta$ are both zero maps, then $\mathfrak{g}^{*} \bowtie_{\sigma, \theta} \mathfrak{f}=\mathfrak{g}^{*} \triangleleft \mathfrak{f}$, defined before. The Lie bialgebra $\mathfrak{g}^{*} \triangleleft_{\sigma, \theta} \mathfrak{f}$ forms an extension

$$
\left(\mathfrak{g}^{*} \bowtie_{\sigma, \theta} \mathfrak{f}\right)=\mathfrak{g}^{*} \rightarrow \mathfrak{g}^{*}{\triangle_{\sigma, \theta}} \mathfrak{f} \rightarrow \mathfrak{f},
$$

together with the natural imbedding from $\mathfrak{g}^{*}$ and the natural projection onto $\mathfrak{f}$. Conversely, the above construction shows that any extension $(\mathfrak{l})=\mathfrak{g}^{*} \rightarrow \mathfrak{l} \rightarrow \mathfrak{f}$ is equivalent to some $\left(\mathfrak{g}^{*} \triangleleft_{\sigma, \theta} \mathfrak{f}\right)$. In fact, as an extension of vector spaces, (ll) may be identified with the trivial one $\mathfrak{g}^{*} \rightarrow \mathfrak{g}^{*} \oplus \mathfrak{f} \rightarrow \mathfrak{f}$. As is easily seen (and well known), the Lie algebra structure on $\mathfrak{l}=\mathfrak{g}^{*} \oplus \mathfrak{f}$ is described as above by some $\rightarrow$ and $\sigma$, where $\rightarrow$ is determined by

$$
a \rightarrow f=[a, f] \quad\left(a \in \mathfrak{f}, f \in \mathfrak{g}^{*}\right)
$$

(the right-hand side denotes the bracket of $\mathfrak{l}$ ). Similarly, since $\left(\mathfrak{l}^{*}\right)$ is a Lie algebra extension of $\mathfrak{g}$ by $\mathfrak{f}^{*}$, the Lie algebra structure on $\mathfrak{l}^{*}=\mathfrak{g} \oplus \mathfrak{f}^{*}$, or dually the Lie coalgebra structure on $\mathfrak{l}$ is described as above by some $\rho$ and $\theta$, where $\rho$ is determined by

$$
\rho(a)=\left(\pi_{2} \otimes \pi_{1}\right) \delta(a) \quad(a \in \mathfrak{f})
$$

with $\pi_{1}, \pi_{2}$ the natural projections $\mathfrak{g}^{*} \leftarrow \mathfrak{g}^{*} \oplus \mathfrak{f} \rightarrow \mathfrak{f}$. The preceding proposition forces $\rightarrow, \rho, \sigma, \theta$ to satisfy the conditions therein, and $(\mathfrak{l})$ is clearly equivalent to $\left(\mathfrak{g}^{*} \triangleleft_{\sigma, \theta} \mathfrak{f}\right)$.

Definition 1.11. Fix a structure $(\rightarrow, \rho)$ of a Singer pair on $\left(\mathfrak{f}, \mathfrak{g}^{*}\right)$. Denote by

$$
\operatorname{Opext}\left(\mathfrak{f}, \mathfrak{g}^{*}\right)=\operatorname{Opext}\left(\mathfrak{f}, \mathfrak{g}^{*}, \rightarrow, \rho\right)
$$

the set of the equivalence classes of all Lie bialgebra extensions $(\mathfrak{l})$ of $\mathfrak{f}$ by $\mathfrak{g}^{*}$ which give rise to $\rightarrow, \rho$ in the way of $(1.9),(1.10)$.

Each Opext $\left(\mathfrak{f}, \mathfrak{g}^{*}\right)$ contains at least one element, the equivalence class of the split extension $\left(\mathfrak{g}^{*} \triangleleft \mathfrak{f}\right)$. The set $\operatorname{Ext}\left(\mathfrak{f}, \mathfrak{g}^{*}\right)$ is the disjoint union of all $\operatorname{Opext}\left(\mathfrak{f}, \mathfrak{g}^{*}, \rightarrow, \rho\right)$, where $(\rightarrow, \rho)$ ranges over the structures of a Singer pair on $\left(\mathfrak{f}, \mathfrak{g}^{*}\right)$.

Proposition 1.12. Fix a structure $(\rightarrow, \rho)$ of a Singer pair on $\left(\mathfrak{f}, \mathfrak{g}^{*}\right)$. Let

$$
\sigma, \sigma^{\prime}: \mathfrak{g} \otimes \wedge^{2} \mathfrak{f} \rightarrow k, \quad \theta, \theta^{\prime}: \wedge^{2} \mathfrak{g} \otimes \mathfrak{f} \rightarrow k
$$

be 2-cocycles for $\mathfrak{g}^{*}$ and for $\mathfrak{f}^{*}$, respectively, such that the pairs $(\sigma, \theta),\left(\sigma^{\prime}, \theta^{\prime}\right)$ both satisfy the equation in Proposition 1.8. The extensions $\left(\mathfrak{g}^{*} \triangleleft_{\sigma, \theta} \mathfrak{f}\right),\left(\mathfrak{g}^{*} \triangleleft_{\sigma^{\prime}, \theta^{\prime}} \mathfrak{f}\right)$ are equivalent to each other if and only if there exists a linear map $\nu: \mathfrak{g} \otimes \mathfrak{f} \rightarrow k$ $(\nu(x \otimes a)$ is written instead as $\nu(x ; a))$ such that

$$
\begin{aligned}
& \sigma(x ; a, b)-\sigma^{\prime}(x ; a, b)=\nu(x \triangleleft a ; b)-\nu(x \triangleleft b ; a)-\nu(x ;[a, b]), \\
& \theta^{\prime}(x, y ; a)-\theta(x, y ; a)=\nu(x ; y \triangleright a)-\nu(y ; x \triangleright a)-\nu([x, y] ; a)
\end{aligned}
$$

for $x, y \in \mathfrak{g}, a, b \in \mathfrak{f}$, where $(\triangleright, \triangleleft)$ is the structure of a matched pair on $(\mathfrak{f}, \mathfrak{g})$ corresponding to $(\rightarrow, \rho)$. 


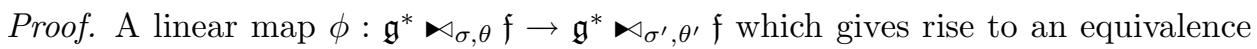
between the two vector space extensions is in the form

$$
\phi(f \oplus a)=(f+\nu a) \oplus a \quad\left(f \in \mathfrak{g}^{*}, a \in \mathfrak{f}\right),
$$

where $\nu: \mathfrak{f} \rightarrow \mathfrak{g}^{*}$ is a linear map. Identifying $\nu$ with

$$
\mathfrak{g} \otimes \mathfrak{f} \rightarrow k, \quad x \otimes a \mapsto\langle x, \nu a\rangle,
$$

we have only to show that $\phi$ is a Lie bialgebra map if and only if $\nu$ satisfies the two equations given above. It follows by a simple computation that $\phi$ is a Lie algebra map if and only if

$$
\sigma(a, b)-\sigma^{\prime}(a, b)=(a-\nu b)-(b \rightarrow \nu a)-\nu[a, b]
$$

for $a, b \in \mathfrak{f}$. This is equivalent to the first equation. One sees that $\phi^{*}: \mathfrak{g} \oplus \mathfrak{f}^{*} \rightarrow \mathfrak{g} \oplus \mathfrak{f}^{*}$ is given by

$$
\phi^{*}(x \oplus p)=x \oplus\left(\nu^{*} x+p\right) \quad\left(x \in \mathfrak{g}, p \in \mathfrak{f}^{*}\right) .
$$

We may identify $\nu^{*}$, too, with the map (1.13). Hence, $\phi$ is a Lie coalgebra map if and only if $\phi^{*}$ is a Lie algebra map from the Lie algebra with the bracket determined by $\rho^{*}, \theta^{\prime}$ to the Lie algebra with the bracket determined by $\rho^{*}, \theta$, which in turn holds if and only if the second equation holds.

In the next section, we will see that the set $\operatorname{Opext}\left(\mathfrak{f}, \mathfrak{g}^{*}\right)$ is in 1-1 correspondence with the first total cohomology group $H^{1}\left(\operatorname{Tot} C_{0}^{\cdot *}\right)$ of a certain double complex $C_{0}^{\cdot}$.

\section{Cohomological Description for lie Bialgebra Extensions}

This section is devoted to giving a Lie bialgebra version of the pioneering work of Kac [Kac, who established the theory of $\mathbb{C}$-Hopf algebra (more precisely, Kac algebra) extensions in the form $\mathbb{C}^{G} \rightarrow A \rightarrow \mathbb{C} F$, where $\mathbb{C} F$ is a finite group algebra and $\mathbb{C}^{G}$ is the dual of such an algebra $\mathbb{C} G$.

Given a Hopf algebra, we denote its coproduct, counit and antipode as usual by $\Delta, \varepsilon$ and $S$, respectively. Let us use the sigma notation due to Sweedler:

$$
\Delta a=\sum a_{(1)} \otimes a_{(2)}, \quad(1 \otimes \Delta) \Delta(a)=\sum a_{(1)} \otimes a_{(2)} \otimes a_{(3)},
$$

and so forth. If the Hopf algebra in question is cocommutative, then the numbering in the subscripts (1), (2), .. is not essential, and so we sometimes omit them and write

$$
\Delta a=\sum_{(a)} a \otimes a, \quad(1 \otimes \Delta) \Delta(a)=\sum_{(a)} a \otimes a \otimes a
$$

and so forth.

Let $H, J$ be cocommutative Hopf algebras possibly of infinite dimension.

Definition 2.1 Kas, Definition IX.2.2]. A pair $(H, J)$ equipped with actions

$$
\triangleright: J \otimes H \rightarrow H, \quad \triangleleft: J \otimes H \rightarrow J
$$

is called a matched pair of (cocommutative) Hopf algebras, if $H$ is a left $J$-module coalgebra under $\triangleright$, if $J$ is a right $H$-module coalgebra under $\triangleleft$, and if

$$
\begin{aligned}
& \text { 1) } x \triangleright a b=\sum\left(x_{(1)} \triangleright a_{(1)}\right)\left(\left(x_{(2)} \triangleleft a_{(2)}\right) \triangleright b\right), \\
& \text { 2) } x y \triangleleft a=\sum\left(x \triangleleft\left(y_{(1)} \triangleright a_{(1)}\right)\right)\left(y_{(2)} \triangleleft a_{(2)}\right)
\end{aligned}
$$

for $x, y \in J, a, b \in H$. 
By a left $J$-module coalgebra, we mean a left $J$-module $H$ such that

$$
\Delta(x \triangleright a)=\sum\left(x_{(1)} \triangleright a_{(1)}\right) \otimes\left(x_{(2)} \triangleright a_{(2)}\right), \quad \varepsilon(x \triangleright a)=\varepsilon(x) \varepsilon(a)
$$

for $x \in J, a \in H$. It is straightforward to see that the conditions given in Definition 2.1 are equivalent to the condition that the tensor product coalgebra $H \otimes J$ forms a bialgebra with unit $1 \otimes 1$, equipped with the product defined by

$$
(a \otimes x)(b \otimes y)=\sum a\left(x_{(1)} \triangleright b_{(1)}\right) \otimes\left(x_{(2)} \triangleleft b_{(2)}\right) y,
$$

where $a \otimes x, b \otimes y \in H \otimes J$. In this case, the bialgebra $H \otimes J$ has an antipode given by

$$
S(a \otimes x)=\sum\left(S\left(x_{(2)}\right) \triangleright S\left(a_{(2)}\right)\right) \otimes\left(S\left(x_{(1)}\right) \triangleleft S\left(a_{(1)}\right)\right)
$$

for $a \otimes x \in H \otimes J$. This Hopf algebra is denoted by $H \bowtie J$, and its element $a \otimes x$ is denoted instead by $a \bowtie x$. See Kas, Theorem IX.2.3].

Let $(H, J, \triangleright, \triangleleft)$ be a matched pair of cocommutative Hopf algebras. Since $H \bowtie J$ is generated by $H=H \otimes k$ and $J=k \otimes J(\subset H \bowtie J)$, a left $H$-and $J$-module $M$ gives rise to a left $H \bowtie J$-module if and only if

$$
x(a m)=\sum\left(x_{(1)} \triangleright a_{(1)}\right)\left(\left(x_{(2)} \triangleleft a_{(2)}\right) m\right)
$$

for all $x \in J, a \in H, m \in M$. A symmetric statement holds for right $H \bowtie J$ modules.

Proposition 2.3. Let $P$ be a left $J$-module, and let $Q$ be a right $H$-module.

1) Regard $H \otimes P$ as a left $H$-module via the left multiplication by $H$ on the factor $H$. Then, $H \otimes P$ is a left $H \bowtie J$-module, equipped with the $J$-action determined by

$$
x(a \otimes p)=\sum\left(x_{(1)} \triangleright a_{(1)}\right) \otimes\left(x_{(2)} \triangleleft a_{(2)}\right) p
$$

for $x \in J, a \otimes p \in H \otimes P$.

2) Regard $Q \otimes J$ as a right $J$-module via the right multiplication by $J$ on the factor $J$. Then, $Q \otimes J$ is a right $H \bowtie J$-module, equipped with the $H$-action determined by

$$
(q \otimes x) a=\sum q\left(x_{(1)} \triangleright a_{(1)}\right) \otimes\left(x_{(2)} \triangleleft a_{(2)}\right)
$$

for $a \in H, q \otimes x \in Q \otimes J$.

3) Regard $Q \otimes J$ further as a left $H \bowtie J$-module by twisting the action via the antipode of $H \bowtie J$, and regard $(Q \otimes J) \otimes(H \otimes P)$ as a left $H \bowtie J$-module via the diagonal action. Regard $(H \bowtie J) \otimes P \otimes Q$ as a left $H \bowtie J$-module via the left multiplication by $H \bowtie J$. Then there is an $H \bowtie J$-linear isomorphism $\beta:(H \bowtie J) \otimes P \otimes Q \rightarrow(Q \otimes J) \otimes(H \otimes P)$ given by

$$
\beta((a \bowtie x) \otimes p \otimes q)=\sum_{(x),(a)} q(S(x) \triangleright S(a)) \otimes(S(x) \triangleleft S(a)) \otimes a \otimes x p
$$

with the abbreviated sigma notation. Hence, $(Q \otimes J) \otimes(H \otimes P)$ is a free left $H \bowtie J$ module in which any basis of the vector space $(Q \otimes k) \otimes(k \otimes P)$ is an $H \bowtie J$-free basis. 
Proof. 1) It is easy to see from (2.1.2) that $H \otimes P$ is a left $J$-module under the $J$ action defined above. Part 1 follows if we compute, for $x \in J, a \in H, b \otimes p \in H \otimes P$,

$$
\begin{aligned}
x(a(b \otimes p)) & =x(a b \otimes p) \\
& =\sum_{(x),(a)}(x \triangleright a b) \otimes(x \triangleleft a b) p \\
& =\sum_{(x),(a)}(x \triangleright a)((x \triangleleft a) \triangleright b) \otimes((x \triangleleft a) \triangleleft b) p \\
& =\sum_{(x),(a)}(x \triangleright a)((x \triangleleft a)(b \otimes p)) .
\end{aligned}
$$

2) Similar.

3) Clearly, $\beta$ is $H \bowtie J$-linear. Define a linear map $\gamma:(Q \otimes J) \otimes(H \otimes P) \rightarrow$ $(H \bowtie J) \otimes P \otimes Q$ by

$$
\gamma(q \otimes x \otimes a \otimes p)=\sum_{(x),(a)}(a \bowtie S(x \triangleleft a)) \otimes(x \triangleleft a) p \otimes q(x \triangleright a) .
$$

We have $\beta \gamma=1$, since

$$
\begin{aligned}
& \beta \gamma(q \otimes x \otimes a \otimes p) \\
& =\sum_{(x),(a)} q(x \triangleright a)((x \triangleleft a) \triangleright S(a)) \otimes(x \triangleleft a S(a)) \otimes a \otimes S(x \triangleleft a)(x \triangleleft a) p \\
& =\sum_{(a)} q(x \triangleright a S(a)) \otimes x \otimes a \otimes p=q \otimes x \otimes a \otimes p .
\end{aligned}
$$

The last equality follows from

$$
x \triangleright 1=\varepsilon(x) 1 \quad(x \in J),
$$

which holds since

$$
1 \bowtie x=(1 \bowtie x)(1 \bowtie 1)=\sum\left(x_{(1)} \triangleright 1\right) \bowtie\left(x_{(2)} \triangleleft 1\right) .
$$

Similarly, we have $\gamma \beta=1$, and hence $\beta$ is an isomorphism. The last assertion holds true since $\beta$ maps $k \otimes P \otimes Q$ onto $(Q \otimes k) \otimes(k \otimes P)$.

Let $\mathfrak{f}$ be a finite-dimensional Lie algebra. The universal enveloping algebra $U \mathfrak{f}$ of $\mathfrak{f}$ has a natural Hopf algebra structure in which every element of $\mathfrak{f}$ is primitive, and hence it is cocommutative (see [Mo, Example 1.5.4]). Let $\mathfrak{g}$ be another Lie algebra of finite dimension.

Proposition 2.4. If $(\mathfrak{f}, \mathfrak{g}, \triangleright, \triangleleft)$ is a matched pair of Lie algebras, the actions $\triangleright$ : $\mathfrak{g} \otimes \mathfrak{f} \rightarrow \mathfrak{f}, \triangleleft: \mathfrak{g} \otimes \mathfrak{f} \rightarrow \mathfrak{g}$ can be extended uniquely to actions

$$
\triangleright: U \mathfrak{g} \otimes U \mathfrak{f} \rightarrow U \mathfrak{f}, \quad \triangleleft: U \mathfrak{g} \otimes U \mathfrak{f} \rightarrow U \mathfrak{g}
$$

with which $(U \mathfrak{f}, U \mathfrak{g})$ is a matched pair of Hopf algebras. Furthermore, the Hopf algebra $U \mathfrak{f} \bowtie U \mathfrak{g}$ constructed from the pair $(U \mathfrak{f}, U \mathfrak{g}, \triangleright, \triangleleft)$ is naturally isomorphic to the universal enveloping algebra $U(\mathfrak{f} \bowtie \mathfrak{g})$ of the Lie algebra $\mathfrak{f} \bowtie \mathfrak{g}$ constructed from the pair $(\mathfrak{f}, \mathfrak{g}, \triangleright, \triangleleft)$. If the characteristic $\operatorname{ch} k$ is zero, every matched pair $(U \mathfrak{f}, U \mathfrak{g})$ is obtained in this way from some matched pair $(\mathfrak{f}, \mathfrak{g})$. 
Proof. The uniqueness of extensions follows from Definition 2.1, since the algebras $U \mathfrak{f}, U \mathfrak{g}$ are generated by $\mathfrak{f}, \mathfrak{g}$, respectively.

We may regard $U \mathfrak{f}, U \mathfrak{g}$ naturally as Hopf subalgebras of $U(\mathfrak{f} \bowtie \mathfrak{g})$, and then by the Poincaré-Birkhoff-Witt theorem the product map $\mu: U \mathfrak{f} \otimes U \mathfrak{g} \rightarrow U(\mathfrak{f} \bowtie \mathfrak{g})$ is a coalgebra isomorphism. Hence there is a unique structure $(\triangleright, \triangleleft)$ of a matched pair on $(U \mathfrak{f}, U \mathfrak{g})$ such that $\mu: U \mathfrak{f} \bowtie U \mathfrak{g} \rightarrow U(\mathfrak{f} \bowtie \mathfrak{g})$ is a Hopf algebra isomorphism. Since $\mu$ induces a Lie algebra isomorphism $(\mathfrak{f} \otimes k) \oplus(k \otimes \mathfrak{g}) \stackrel{\sim}{\rightarrow} \mathfrak{f} \bowtie \mathfrak{g}$, the structure $(\triangleright, \triangleleft)$ just obtained is an extension of the original $(\triangleright, \triangleleft)$ associated with $(\mathfrak{f}, \mathfrak{g})$.

Suppose $\operatorname{ch} k=0$. Given a matched pair $(U \mathfrak{f}, U \mathfrak{g})$, construct the Hopf algebra $U \mathfrak{f} \bowtie U \mathfrak{g}$. By [Mo Proposition 5.5.3 2)], the Lie algebra $P(U \mathfrak{f} \bowtie U \mathfrak{g})$ of the primitive elements in $U \mathfrak{f} \bowtie U \mathfrak{g}$ equals $(\mathfrak{f} \otimes k) \oplus(k \otimes \mathfrak{g})$, which gives a matched pair $(\mathfrak{f}, \mathfrak{g})$ of Lie algebras. This pair induces the given pair $(U \mathfrak{f}, U \mathfrak{g})$, since one sees that $U \mathfrak{f} \bowtie U \mathfrak{g} \cong U(\mathfrak{f} \bowtie \mathfrak{g})$.

Example 2.5. Let $(\mathfrak{f}, \mathfrak{g})=(k a, k x)$ be the matched pair of Lie algebras given in Example 1.2, with the structure $(\triangleright, \triangleleft)$ defined in (1.3). Then, $U \mathfrak{f}=k[a], U \mathfrak{g}=k[x]$, the polynomial algebras. Define module actions $\triangleright: k[x] \otimes k[a] \rightarrow k[a], \triangleleft: k[x] \otimes$ $k[a] \rightarrow k[x]$ by

$$
\begin{array}{ll}
x \triangleright 1=0, & x \triangleright a^{n}=\sum_{i=0}^{n-1}\left(\begin{array}{c}
n \\
i+1
\end{array}\right) s t^{i} a^{n-i} \quad(n>0), \\
1 \triangleleft a=0, & x^{n} \triangleleft a=\sum_{i=0}^{n-1}\left(\begin{array}{c}
n \\
i+1
\end{array}\right) s^{i} t^{n-i} \quad(n>0) .
\end{array}
$$

Then an induction shows that these are unique extensions of the Lie actions $\triangleright, \triangleleft$, with which $(U \mathfrak{f}, U \mathfrak{g})$ is a matched pair of Hopf algebras.

In the following, we fix a matched pair $(\mathfrak{f}, \mathfrak{g}, \triangleright, \triangleleft)$ of Lie algebras, and we let $(U \mathfrak{f}, U \mathfrak{g}, \triangleright, \triangleleft)$ be the induced matched pair of Hopf algebras. We naturally identify

$$
U \mathfrak{f} \bowtie U \mathfrak{g}=U(\mathfrak{f} \bowtie \mathfrak{g}) .
$$

Define $V_{p}(\mathfrak{f})=U \mathfrak{f} \otimes \wedge^{p} \mathfrak{f}\left(\wedge^{p} \mathfrak{f}\right.$ denotes the $p$ th-exterior product of $\left.\mathfrak{f}\right)$, and write $u\left\langle a_{1}, \ldots, a_{p}\right\rangle$ for $u \otimes\left(a_{1} \wedge \cdots \wedge a_{p}\right) \in V_{p}(\mathfrak{f})$. Let

$$
V .(\mathfrak{f})=0 \leftarrow V_{0}(\mathfrak{f}) \stackrel{\partial}{\leftarrow} V_{1}(\mathfrak{f}) \stackrel{\partial}{\leftarrow} V_{2}(\mathfrak{f}) \stackrel{\partial}{\leftarrow} \cdots
$$

be the Chevalley-Eilenberg complex $\mathrm{CE}$, Chap. XIII, Sect. 7], We, Definition 7.7.1], where the differentials $\partial: V_{p}(\mathfrak{f}) \rightarrow V_{p-1}(\mathfrak{f})$ are given by

$$
\begin{aligned}
\partial\left(u\left\langle a_{1}, \ldots, a_{p}\right\rangle\right)=\sum_{i=1}^{p}(-1)^{i+1} u a_{i}\left\langle a_{1}, \ldots, \hat{a_{i}}, \ldots, a_{p}\right\rangle \\
\quad+\sum_{i<j}(-1)^{i+j} u\left\langle\left[a_{i}, a_{j}\right], a_{1}, \ldots, \hat{a_{i}}, \ldots, \hat{a_{j}}, \ldots, a_{p}\right\rangle
\end{aligned}
$$

(the notation $\hat{a_{i}}$ denots an omitted term). The counit $\varepsilon: V_{0}(\mathfrak{f})=U \mathfrak{f} \rightarrow k$ of $U \mathfrak{f}$ gives a free resolution $V .(\mathfrak{f}) \rightarrow k \rightarrow 0$ of the trivial left $U \mathfrak{f}$-module $k$ CE, Chap. XIII, Theorem 7.1], [We Theorem 7.7.2]. We define a left $\mathfrak{g}$-Lie module structure on $\wedge^{p} \mathfrak{f}$ by

$$
x\left\langle a_{1}, \ldots, a_{p}\right\rangle=\sum_{i=1}^{p}\left\langle a_{1}, \ldots, x \triangleright a_{i}, \ldots, a_{p}\right\rangle \quad(x \in \mathfrak{g}) .
$$


This is equivalent to saying that we define a left $U \mathfrak{g}$-module structure on $\wedge^{p} \mathfrak{f}$ by

$$
u\left\langle a_{1}, \ldots, a_{p}\right\rangle=\sum_{(u)}\left\langle u_{(1)} \triangleright a_{1}, \ldots, u_{(p)} \triangleright a_{p}\right\rangle \quad(u \in U \mathfrak{g}) .
$$

Regard each $V_{p}(\mathfrak{f})$ as a left $U(\mathfrak{f} \bowtie \mathfrak{g})$-module by applying Proposition 2.31 ) to $H=U \mathfrak{f}, J=U \mathfrak{g}, P=\wedge^{p} \mathfrak{f}$, and suppose $k$ to be the trivial left $U(\mathfrak{f} \bowtie \mathfrak{g})$-module.

Lemma 2.6. The differentials $\partial$ and the augmentation $\varepsilon$ are all $U(\mathfrak{f} \bowtie \mathfrak{g})$-linear.

Proof. The assertion for $\varepsilon$ is clear.

Let $x \in \mathfrak{g}, a_{i} \in \mathfrak{f}$. We will show that

$$
\partial\left\{x\left(u\left\langle a_{1}, \ldots, a_{p}\right\rangle\right)\right\}=x \partial\left(u\left\langle a_{1}, \ldots, a_{p}\right\rangle\right)
$$

by induction on the length $l$ in the expression $u=b_{1} \cdots b_{l}$ with $b_{i} \in \mathfrak{f}$.

Suppose $l=0$, so $u=1$. We compute the right-hand side of (2.7) to obtain

$$
\begin{aligned}
& \sum_{i}(-1)^{i+1}\left(x \triangleright a_{i}\right)\left\langle a_{1}, \ldots, a_{p}\right\rangle+\sum_{i}(-1)^{i+1}\left(x \triangleleft a_{i}\right)\left\langle a_{1}, \ldots, a_{p}\right\rangle \\
& +\sum_{i}(-1)^{i+1} a_{i}\left(x\left\langle a_{1}, \ldots, a_{p}\right\rangle\right)+\sum_{i<j}(-1)^{i+j}\left\langle x \triangleright\left[a_{i}, a_{j}\right], a_{1}, \ldots, a_{p}\right\rangle \\
& +\sum_{i<j, k \neq i, j}(-1)^{i+j}\left\langle\left[a_{i}, a_{j}\right], a_{1}, \ldots, x \triangleright a_{k}, \ldots, a_{p}\right\rangle .
\end{aligned}
$$

From the equation (1.1.1), we see that the sum of the fourth and the second terms equals

$$
\begin{aligned}
& \sum_{i<j}(-1)^{i+j}\left\langle\left[x \triangleright a_{i}, a_{j}\right], a_{1}, \ldots, a_{p}\right\rangle \\
& +\sum_{i<j}(-1)^{i+j}\left\langle\left[a_{i}, x \triangleright a_{j}\right], a_{1}, \ldots, a_{p}\right\rangle \\
& +\sum_{i<j}(-1)^{i}\left\langle a_{1}, \ldots, \hat{a_{i}}, \ldots,\left(x \triangleleft a_{i}\right) \triangleright a_{j}, \ldots, a_{p}\right\rangle \\
& +\sum_{i<j}(-1)^{j}\left\langle a_{1}, \ldots,\left(x \triangleleft a_{j}\right) \triangleright a_{i}, \ldots, \hat{a_{j}}, \ldots, a_{p}\right\rangle \\
& +\sum_{i}(-1)^{i+1}\left(x \triangleleft a_{i}\right)\left\langle a_{1}, \ldots, \hat{a_{i}}, \ldots, a_{p}\right\rangle,
\end{aligned}
$$

in which the sum of the last three terms is clearly zero. Now, one computes the left-hand side of (2.7) to see that the desired equality holds.

Suppose that the equation (2.7) holds, that is, $\partial(x \sigma)=x \partial \sigma$ with $\sigma=u\left\langle a_{1}, \ldots, a_{p}\right\rangle$. Then for $b \in \mathfrak{f}$, we have

$$
\begin{aligned}
\partial(x(b \sigma)) & =\partial\{(x \triangleright b+x \triangleleft b+b x) \sigma\} \\
& =(x \triangleright b+x \triangleleft b+b x) \partial \sigma=x b \partial \sigma=x \partial(b \sigma),
\end{aligned}
$$

which completes the induction.

Define $V_{p}^{\prime}(\mathfrak{g})=\wedge^{p} \mathfrak{g} \otimes U \mathfrak{g}$, and let

$$
V_{.}^{\prime}(\mathfrak{g})=0 \longleftarrow V_{0}^{\prime}(\mathfrak{g}) \stackrel{\partial^{\prime}}{\longleftarrow} V_{1}^{\prime}(\mathfrak{g}) \stackrel{\partial^{\prime}}{\longleftarrow} V_{2}^{\prime}(\mathfrak{g}) \stackrel{\partial^{\prime}}{\longleftarrow} \cdots
$$

be the right version of the Chevalley-Eilenberg complex, which is a free resolution of the trivial right $U \mathfrak{g}$-module $k$, given the counit $\varepsilon: V_{0}^{\prime}(\mathfrak{g})=U \mathfrak{g} \rightarrow k$ of $U \mathfrak{g}$ as 
augmentation. The mirror argument including Proposition 2.32 ) leads us to see that each $V_{p}^{\prime}(\mathfrak{g})$ is a right $U(\mathfrak{f} \bowtie \mathfrak{g})$-module and $\partial^{\prime}$, $\varepsilon$ are $U(\mathfrak{f} \bowtie \mathfrak{g})$-linear. By tensoring $V^{\prime}(\mathfrak{g})$ with $V .(\mathfrak{f})$, we obtain the double complex

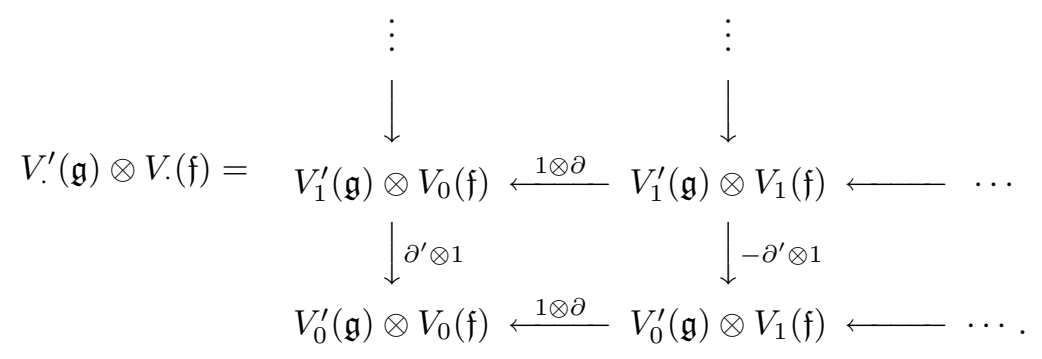

Here each row has the differentials $1 \otimes \partial$, while the $p$ th column has the differentials $(-1)^{p} \partial^{\prime} \otimes 1$. As in Proposition 2.33$)$, we regard $V_{p}^{\prime}(\mathfrak{g})$ as a left $U(\mathfrak{f} \bowtie \mathfrak{g})$-module by twisting the action via the antipode, and also regard $V_{q}^{\prime}(\mathfrak{g}) \otimes V_{p}(\mathfrak{f})$ as a left $U(\mathfrak{f} \bowtie \mathfrak{g})$-module via the diagonal action.

Proposition 2.8. The total complex of $V^{\prime}(\mathfrak{g}) \otimes V$.(f) is a free resolution of the trivial $U(\mathfrak{f} \bowtie \mathfrak{g})$-module $k$, equipped with $\varepsilon \otimes \varepsilon: V_{0}^{\prime}(\mathfrak{g}) \otimes V_{0}(\mathfrak{f})=U \mathfrak{g} \otimes U \mathfrak{f} \rightarrow k$ as augmentation.

Proof. Compute the $E^{2}$-term of the spectral sequence arising from the first filtration of the total complex. Then one has, easily,

$$
E_{p q}^{2} \cong \begin{cases}k, & \text { if } p=q=0 \\ 0, & \text { otherwise. }\end{cases}
$$

It follows from $[\mathrm{R}$, Lemma 11.20] that the total complex is a resolution of $k$, whose augmentation is $\varepsilon \otimes \varepsilon$ since the isomorphism $E_{00}^{2} \cong k$ is induced from it. By Proposition 2.33 ), the resolution just obtained is free.

Apply $\operatorname{Hom}_{U(\mathfrak{f} \bowtie \mathfrak{g})}(, k)$ to $V^{\prime}(\mathfrak{g}) \otimes V .(\mathfrak{f})$. Then, since the last statement in Proposition 2.33 ) allows us to identify

$$
\operatorname{Hom}_{U(\mathfrak{f} \bowtie \mathfrak{g})}\left(V_{q}^{\prime}(\mathfrak{g}) \otimes V_{p}(\mathfrak{f}), k\right)=\operatorname{Hom}\left(\wedge^{q} \mathfrak{g} \otimes \wedge^{p} \mathfrak{f}, k\right),
$$

we have the following double complex of vector spaces:

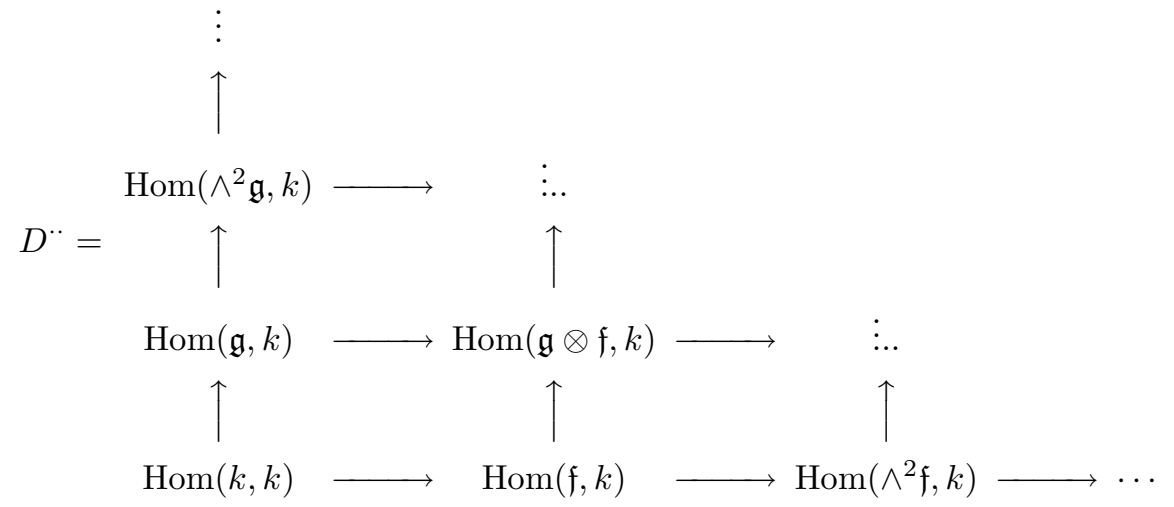


Furthermore, delete from $D^{*}$ its edge complexes:

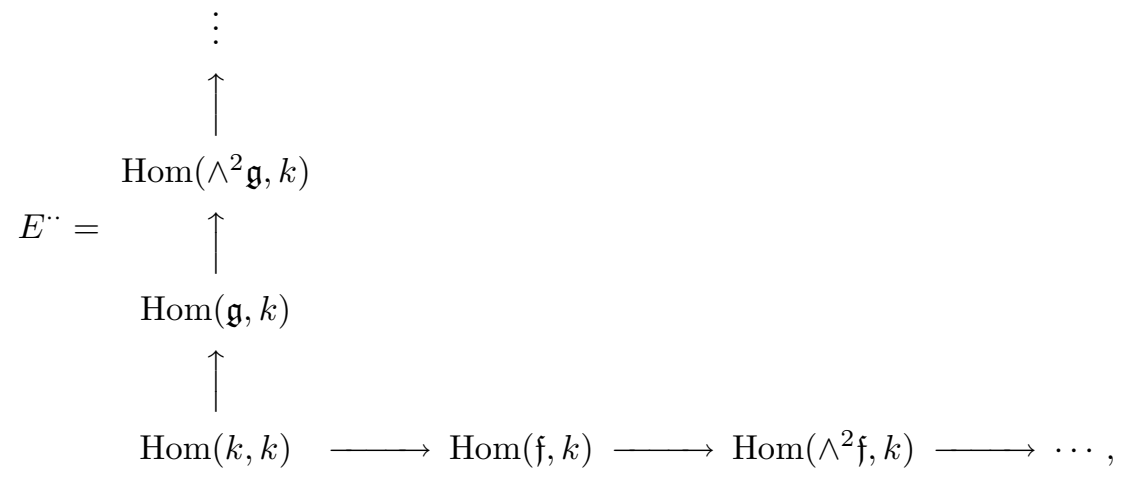

which consist of the standard complexes for computing the cohomology groups $H^{\cdot}(\mathfrak{f}, k), H^{\cdot}(\mathfrak{g}, k)$ of Lie algebras. Then we have the double complex

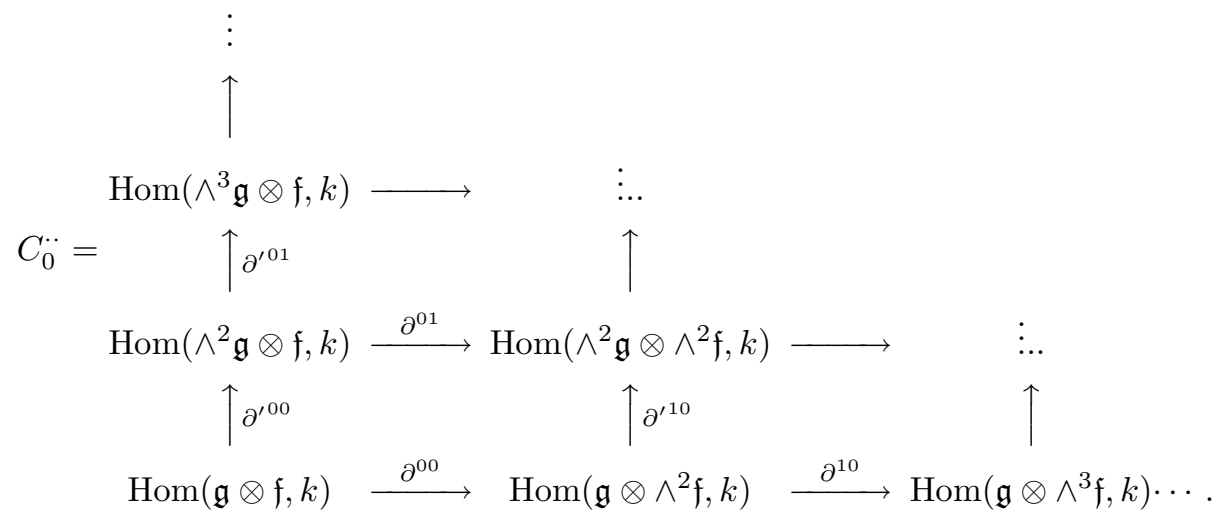

Denote by Tot $C_{0}$ the total complex of $C_{0}$.

Let Aut $\left(\mathfrak{g}^{*} \bowtie \mathfrak{f}\right)$ denote the group of the auto-equivalences of the split extension $\left(\mathfrak{g}^{*} \bowtie \mathfrak{f}\right)$.

Proposition 2.9. Let $\left(\mathfrak{f}, \mathfrak{g}^{*}, \rightarrow, \rho\right)$ be the Singer pair corresponding to $(\mathfrak{f}, \mathfrak{g}, \triangleright, \triangleleft)$.

1) There is a natural isomorphism of groups

$$
H^{0}\left(\operatorname{Tot} C_{0}^{\cdot \cdot}\right) \cong \operatorname{Aut}\left(\mathfrak{g}^{*} \bowtie \mathfrak{f}\right) .
$$

2) There is a natural bijection between sets

$$
H^{1}\left(\operatorname{Tot} C_{0}^{\cdot}\right) \cong \operatorname{Opext}\left(\mathfrak{f}, \mathfrak{g}^{*}, \rightarrow, \rho\right)
$$

Proof. 1) Let $\nu \in \operatorname{Hom}(\mathfrak{g} \otimes \mathfrak{f}, k)$. The differentials $\partial^{00}, \partial^{\prime 00}$ in $C_{0}^{\cdot *}$ are given by

$$
\begin{aligned}
& \partial^{00} \nu(x ; a, b)=\nu(x \triangleleft a ; b)-\nu(x \triangleleft b ; a)-\nu(x ;[a, b]), \\
& \partial^{\prime 00} \nu(x, y ; a)=-\nu(x ; y \triangleright a)+\nu(y ; x \triangleright a)+\nu([x, y] ; a)
\end{aligned}
$$

for $x, y \in \mathfrak{g}, a, b \in \mathfrak{f}$. Suppose that $\sigma=\sigma^{\prime}=0, \theta=\theta^{\prime}=0$ in Proposition 1.12. Then one sees that $\nu \mapsto(f \oplus a \mapsto(f+\nu a) \oplus a)$ gives an isomorphism $H^{0}\left(\operatorname{Tot} C_{0}^{\cdot *}\right) \cong \operatorname{Aut}\left(\mathfrak{g}^{*} \bowtie \mathfrak{f}\right)$. 
2) Suppose $\sigma \in \operatorname{Hom}\left(\mathfrak{g} \otimes \wedge^{2} \mathfrak{f}, k\right), \theta \in \operatorname{Hom}\left(\wedge^{2} \mathfrak{g} \otimes \mathfrak{f}, k\right)$. The differentials $\partial^{\prime 10}, \partial^{01}$ in $C_{0}^{*}$ are given by

$$
\begin{aligned}
\partial^{\prime 10} \sigma(x, y ; a, b) & =\sigma(x ; y \triangleright a, b)+\sigma(x ; a, y \triangleright b)-(x \leftrightarrow y)-\sigma([x, y] ; a, b), \\
\partial^{01} \theta(x, y ; a, b) & =\theta(x, y \triangleleft a ; b)+\theta(x \triangleleft a, y ; b)-(a \leftrightarrow b)-\theta(x, y ;[a, b])
\end{aligned}
$$

for $x, y \in \mathfrak{g}, a, b \in \mathfrak{f}$. Hence the equation given in Proposition 1.8 is equivalent to the equation $\partial^{\prime 10} \sigma+\partial^{01} \theta=0$. Notice further that $\sigma \in \operatorname{Ker} \partial^{10}$ (respectively, $\theta \in \operatorname{Ker} \partial^{\prime 01}$ ) if and only if $\sigma$ (respectively, $\theta$ ) is a 2-cocycle for $\mathfrak{g}^{*}$ (respectively, for $\left.\mathfrak{f}^{*}\right)$. It follows from Proposition 1.8 that $\sigma, \theta$ well define the Lie bialgebra $\mathfrak{g}^{*} \triangleleft_{\sigma, \theta} \mathfrak{f}$ if and only if $(\sigma, \theta)$ is in $Z^{1}\left(\operatorname{Tot} C_{0}^{\cdot}\right)$, the 1-cocycles in $\operatorname{Tot} C_{0}^{*}$. This implies that we have a correspondence $Z^{1}\left(\operatorname{Tot} C_{0}^{*}\right) \rightarrow \operatorname{Opext}\left(\mathfrak{f}, \mathfrak{g}^{*}\right)$ given by $(\sigma, \theta) \mapsto\left(\mathfrak{g}^{*} \triangleleft_{\sigma, \theta} \mathfrak{f}\right)$, which is a surjection by the argument after Proposition 1.8. Moreover, Proposition 1.12 shows that the Lie bialgebra extensions $\left(\mathfrak{g}^{*} \bowtie_{\sigma, \theta} \mathfrak{f}\right)$ and $\left(\mathfrak{g}^{*} \bowtie_{\sigma^{\prime}, \theta^{\prime}} \mathfrak{f}\right)$ are equivalent if and only if $(\sigma, \theta)$ and $\left(\sigma^{\prime}, \theta^{\prime}\right)$ are cohomologous in Tot $C_{0}^{\cdot}$. Therefore we have the desired bijection.

Equip Opext $\left(\mathfrak{f}, \mathfrak{g}^{*}, \rightarrow, \rho\right)$ uniquely with an abelian group structure so that the bijection in Part 2 of the preceding proposition is an isomorphism of groups.

Theorem 2.10. Let $\left(\mathfrak{f}, \mathfrak{g}^{*}, \rightarrow, \rho\right)$ be as above. (Then one can form the Lie algebra $\mathfrak{f} \bowtie \mathfrak{g}$ from the corresponding $(\mathfrak{f}, \mathfrak{g}, \triangleright, \triangleleft)$.) We have an exact sequence

$$
\begin{aligned}
0 & \rightarrow H^{1}(\mathfrak{f} \bowtie \mathfrak{g}, k) \rightarrow H^{1}(\mathfrak{f}, k) \oplus H^{1}(\mathfrak{g}, k) \rightarrow \operatorname{Aut}\left(\mathfrak{g}^{*} \bowtie \mathfrak{f}\right) \\
& \rightarrow H^{2}(\mathfrak{f} \bowtie \mathfrak{g}, k) \rightarrow H^{2}(\mathfrak{f}, k) \oplus H^{2}(\mathfrak{g}, k) \rightarrow \operatorname{Opext}\left(\mathfrak{f}, \mathfrak{g}^{*}, \rightarrow, \rho\right) \\
& \rightarrow H^{3}(\mathfrak{f} \bowtie \mathfrak{g}, k) \rightarrow H^{3}(\mathfrak{f}, k) \oplus H^{3}(\mathfrak{g}, k),
\end{aligned}
$$

where $H \cdot$ indicates the cohomology group of Lie algebras with coefficients the trivial Lie module $k$.

Proof. We know that

$$
\begin{aligned}
& H^{n}\left(\operatorname{Tot} D^{*}\right)=H^{n}(\mathfrak{f} \bowtie \mathfrak{g}, k) \quad(n \geq 0), \\
& H^{n}\left(\operatorname{Tot} E^{*}\right)=H^{n}(\mathfrak{f}, k) \oplus H^{n}(\mathfrak{g}, k) \quad(n>0) .
\end{aligned}
$$

Shift the dimension of $C_{0} \cdot$ to obtain $C_{0}^{\cdot-1, \cdot-1}$, and fill the now empty edges with zeros. Then, $C_{0}^{-1,-1}$ is a double subcomplex of $D^{*}$ with quotient $E^{*}$. Thus we have the short exact sequence

$$
0 \rightarrow \operatorname{Tot} C_{0}^{\cdot-1, \cdot-1} \rightarrow \operatorname{Tot} D^{*} \rightarrow \operatorname{Tot} E^{\cdot *} \rightarrow 0
$$

consisting of the total complexes. Consider the long exact cohomology sequence arising from this, and apply Proposition 2.9 and the two equations above. Then the required exact sequence follows.

We may regard the last five terms in the exact sequence just obtained as a Lie bialgebra version of the Kac exact sequence [Kac, (3.14)]. See also [M, Appendix].

Notice that in the exact sequence above, the maps $H^{n}(\mathfrak{f} \bowtie \mathfrak{g}, k) \rightarrow H^{n}(\mathfrak{f}, k) \oplus$ $H^{n}(\mathfrak{g}, k)(n=1,2,3)$ are induced from the restriction maps for $\mathfrak{f} \subset \mathfrak{f} \bowtie \mathfrak{g} \supset \mathfrak{g}$.

Corollary 2.11. Suppose $\operatorname{ch} k=0$. Let $\left(\mathfrak{f}, \mathfrak{g}^{*}, \rightarrow, \rho\right)$ be as above. If either 1$) \mathfrak{f}$ is semisimple and $\rightarrow$ is zero, or 2) $\mathfrak{g}$ is semisimple and $\rho$ is zero, then the groups $\operatorname{Aut}\left(\mathfrak{g}^{*} \bowtie \mathfrak{f}\right)$ and $\operatorname{Opext}\left(\mathfrak{f}, \mathfrak{g}^{*}\right)$ are both trivial. 
Proof. By symmetry we may suppose 1). Then $\mathfrak{f}$ is an ideal of $\mathfrak{f} \bowtie \mathfrak{g}=\mathfrak{f} \rtimes \mathfrak{g}$, and $\mathfrak{f} \rtimes \mathfrak{g} / \mathfrak{f} \cong \mathfrak{g}$. Since $H^{n}(\mathfrak{f}, k)=0$ for $n=1,2$ by the Whitehead lemmas HS Chap. VII, Sect.6], the conclusion will follow from Theorem 2.10 if we show that the restriction maps $H^{n}(\mathfrak{f} \rtimes \mathfrak{g}, k) \rightarrow H^{n}(\mathfrak{g}, k)(n=1,2)$ are isomorphisms and that the map $H^{3}(\mathfrak{f} \rtimes \mathfrak{g}, k) \rightarrow H^{3}(\mathfrak{f}, k) \oplus H^{3}(\mathfrak{g}, k)$ is an injection.

In general, let $\mathfrak{h}$ be a Lie algebra and let $\mathfrak{a}$ be its ideal. Given a left $\mathfrak{h}$-Lie module $M$, suppose that $H^{n}(\mathfrak{a}, M)=0$ for $0<n<m$, where $m$ is a positive integer. The Hochschild-Serre spectral sequence [CE, Item(6), p.351] implies that the inflation maps $H^{n}\left(\mathfrak{h} / \mathfrak{a}, M^{\mathfrak{a}}\right) \rightarrow H^{n}(\mathfrak{h}, M)(n<m)$ are isomorphisms and that the sequence

$$
0 \rightarrow H^{m}\left(\mathfrak{h} / \mathfrak{a}, M^{\mathfrak{a}}\right) \stackrel{\inf }{\longrightarrow} H^{m}(\mathfrak{h}, M) \stackrel{\text { res }}{\longrightarrow} H^{m}(\mathfrak{a}, M)
$$

is exact, where $M^{\mathfrak{a}}=\{m \in M \mid a m=0$ for all $a \in \mathfrak{a}\}$.

Suppose $\mathfrak{h}=\mathfrak{f} \rtimes \mathfrak{g}, \mathfrak{a}=\mathfrak{f}, M=k$. By the Whitehead lemmas we can apply the results for $m=3$. Notice that the composite

$$
H^{\cdot}(\mathfrak{g}, k) \stackrel{\inf }{\longrightarrow} H^{\cdot}(\mathfrak{f} \rtimes \mathfrak{g}, k) \stackrel{\text { res }}{\longrightarrow} H^{\cdot}(\mathfrak{g}, k)
$$

is the identity map. Then the desired conclusions follow immediately.

\section{Hopf Algebra Extensions}

Let $H, K$ be Hopf algebras, where $H$ is cocommutative and $K$ is commutative.

Definition 3.1 (cf. [Hf, Definition 3.1]). A cleft extension of $H$ by $K$ is a sequence $(A)=K \stackrel{\iota}{\rightarrow} A \stackrel{\pi}{\rightarrow} H$ of Hopf algebras and Hopf algebra maps such that there is a left $K$-linear and right $H$-colinear isomorphism $\zeta: A \stackrel{\sim}{\rightarrow} K \otimes H$, where $A$ is regarded as a left $K$-module along $\iota$ and as a right $H$-comodule along $\pi$ (necessarily, $\iota$ is an injection and $\pi$ is a surjection). Two such extensions $(A),\left(A^{\prime}\right)$ are equivalent if there is a Hopf algebra map (necessarily, an isomorphism) $\phi: A \rightarrow A^{\prime}$ which makes the following diagram commute:

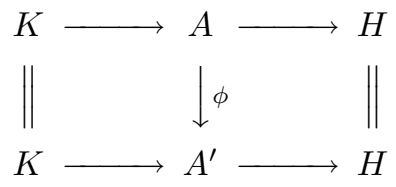

Denote by

$$
\operatorname{Ext}(H, K)
$$

the set of the equivalence classes of all cleft extensions of $H$ by $K$.

Remark 3.2. An isomorphism $\zeta$ as above can be chosen further to be unitary and counitary, that is, $\zeta(1)=1 \otimes 1,(\varepsilon \otimes \varepsilon) \zeta=\varepsilon$ (see [MD, Remark 1.8]).

Definition 3.3. A pair $(H, K)$ eqipped with an action and a coaction,

$$
\rightarrow: H \otimes K \rightarrow K \quad \text { and } \quad \rho: H \rightarrow H \otimes K, \quad \rho a=\sum a_{H} \otimes a_{K},
$$

is called a Singer pair of Hopf algebras, if $K$ is a left $H$-module algebra under $\rightarrow$, if $H$ is a right $K$-comodule coalgebra under $\rho$, and if

$$
\begin{aligned}
& \text { 1) } \rho(a b)=\sum \rho\left(a_{(1)}\right)\left(b_{H} \otimes\left(a_{(2)} \rightarrow b_{K}\right)\right), \\
& \text { 2) } \Delta(a \rightarrow f)=\sum\left(a_{(1) H} \rightarrow f_{(1)}\right) \otimes a_{(1) K}\left(a_{(2)} \rightarrow f_{(2)}\right)
\end{aligned}
$$

for $a, b \in H, f \in K$. 
By a left $H$-module algebra, we mean a left $H$-module $K$ such that

$$
a \rightarrow f g=\sum\left(a_{(1)} \rightarrow f\right)\left(a_{(2)} \rightarrow g\right), \quad a \rightarrow 1=\varepsilon(a) 1
$$

for $a \in H, f, g \in K$. By a right $K$-comodule coalgebra, we mean a right $K$ comodule $H$ such that

$$
\sum \Delta\left(a_{H}\right) \otimes a_{K}=\sum a_{(1) H} \otimes a_{(2) H} \otimes a_{(1) K} a_{(2) K}, \quad \sum \varepsilon\left(a_{H}\right) a_{K}=\varepsilon(a) 1
$$

for $a \in H$.

The above notion was defined first by Singer $[\mathrm{S}]$ in the graded case, and later by Takeuchi $[\mathrm{T}]$ in the ungraded case; they both called it an abelian matched pair. We propose the term given above in parallel with the case of Lie bialgebras. See Definition 1.5.

A cleft extension $(A)$ of $H$ by $K$ gives rise to a structure $(-, \rho)$ of a Singer pair on $(H, K)$, as follows. First, choose such an isomorphism $\zeta: A \stackrel{\sim}{\rightarrow} K \otimes H$ as in Definition 3.1, and define a right $H$-colinear map $\gamma: H \rightarrow A$ and a left $K$-linear map $\omega: A \rightarrow K$ by

$$
\begin{aligned}
& \gamma a=\zeta^{-1}(1 \otimes a) \quad(a \in H), \\
& \omega u=(1 \otimes \varepsilon) \zeta(u) \quad(u \in A),
\end{aligned}
$$

respectively. Then it is seen that these are invertible under the convolution product * [Mo, Definition 1.4.1]. Next, one can define uniquely an action $\rightarrow: H \otimes K \rightarrow K$ and a coaction $\rho: H \rightarrow H \otimes K$ so that

$$
\begin{aligned}
\iota(a \rightarrow f) & =\sum \gamma\left(a_{(1)}\right) \iota(f) \gamma^{-1}\left(a_{(2)}\right) \quad(a \in H, f \in K), \\
\rho \pi(u) & =\sum \pi\left(u_{(2)}\right) \otimes \omega^{-1}\left(u_{(1)}\right) \omega\left(u_{(3)}\right) \quad(u \in A),
\end{aligned}
$$

where $\gamma^{-1}, \omega^{-1}$ denote the convolution-inverses of $\gamma, \omega$, respectively. Finally, we see that $\rightarrow, \rho$ are independent of the choice of $\zeta$, and that these make $(H, K)$ into a Singer pair of Hopf algebras. See [Hf, Sect. 3].

For a Singer pair $(H, K, \rightarrow, \rho)$ of Hopf algebras, we denote by

$$
\operatorname{Opext}(H, K)=\operatorname{Opext}(H, K, \rightarrow, \rho)
$$

the set of the equivalence classes of all cleft extensions that give rise to $\rightarrow, \rho$ in the way described above. It is shown in [Hf, Proposition 5.11] that Opext $(H, K)$ forms an abelian group with respect to the bi-tensor product. The unit is the equivalence class of the split extension $(K \# H)$ whose middle term is the bismash product $K \# H$ [T p. 849] with respect to $\rightarrow, \rho$ (see the proof of Proposition 3.11 below).

The set $\operatorname{Ext}(H, K)$ is the disjoint union of all $\operatorname{Opext}(H, K, \rightarrow, \rho)$, where $(-, \rho)$ ranges over the structures of a Singer pair on $(H, K)$.

Following [S], we will give a cohomological description of $\operatorname{Opext}(H, K)$ (see also [A, Sect. 5]). Fix a Singer pair $(H, K, \rightarrow, \rho)$ of Hopf algebras.

Definition 3.4. We define a category $\mathcal{C}=\mathcal{C}(H, K, \rightarrow, \rho)$, as follows. An object of $\mathcal{C}$ is a left $H$-module $M$ equipped with a right $K$-comodule structure $\lambda: M \rightarrow M \otimes K$, $\lambda m=\sum m_{(0)} \otimes m_{(1)}$, such that

$$
\lambda(a m)=\sum a_{(1) H} m_{(0)} \otimes a_{(1) K}\left(a_{(2)} \rightarrow m_{(1)}\right)
$$

for $a \in H, m \in M$. A morphism in $\mathcal{C}$ is an $H$-linear and $K$-colinear map. 
One sees that the category $\mathcal{C}$ is abelian. The left $H$-module $H$ equipped with $\rho$ and the left $H$-module $(K, \rightarrow)$ equipped with $\Delta$ are both objects in $\mathcal{C}$. Notice also that $k$ is an object in $\mathcal{C}$, equipped with the trivial $H$-action and $K$-coaction.

Lemma 3.5. Let $P$ be a right $K$-comodule. Regard $F(P):=H \otimes P$ as a left $H$ module via the left multiplication by $H$ on the factor $H$, and as a right $K$-comodule via the coaction $F(P) \rightarrow F(P) \otimes K$ given by

$$
a \otimes p \mapsto \sum a_{(1) H} \otimes p_{(0)} \otimes a_{(1) K}\left(a_{(2)} \rightarrow p_{(1)}\right) .
$$

Then, $F(P) \in \mathcal{C}$. Furthermore, $F$ defines a functor from the category Comod- $K$ of right $K$-comodules to $\mathcal{C}$, which is left adjoint to the forgetful functor $U: \mathcal{C} \rightarrow$ Comod- $K$.

Proof. It is verified directly that $F(P) \in \mathcal{C}$. The last statement follows, since we have a familiar isomorphism

$$
\operatorname{Hom}_{H}^{K}(F(P), M) \cong \operatorname{Hom}^{K}(P, U(M))
$$

for $P \in \operatorname{Comod}-K, M \in \mathcal{C}$, which is given by $\phi \mapsto(p \mapsto \phi(1 \otimes p))$. Here, $\operatorname{Hom}_{H}^{K}$ (respectively, $\operatorname{Hom}^{K}$ ) indicates the $H$-linear and $K$-colinear (respectively, the $K$ colinear) maps.

We have the composite $F U: \mathcal{C} \rightarrow \mathcal{C}$ of the functors, which will be denoted simply by $F$ in the sequel. Define the natural transformations $\varepsilon: F \rightarrow I, \delta: F \rightarrow F^{2}$, where $I$ is the identity functor, by

$$
\begin{aligned}
& \varepsilon_{M}: H \otimes M \rightarrow M, \quad \varepsilon_{M}(a \otimes m)=a m, \\
& \delta_{M}: H \otimes M \rightarrow H \otimes H \otimes M, \quad \delta_{M}(a \otimes m)=a \otimes 1 \otimes m
\end{aligned}
$$

for $M \in \mathcal{C}$. Then one sees from $\underline{\mathrm{We}}, 8.6 .2, \mathrm{p} .280]$ that $(F, \varepsilon, \delta)$ forms a cotriple on $\mathcal{C}$. Hence, for each $M \in \mathcal{C}$, we have simplical object $\Phi .(M):=F^{++1}(M)$ in $\mathcal{C}$, which is accompanied by the face and degeneracy operators determined from $\varepsilon, \delta$. We need in particular $\Phi .(k)$, which looks like

$$
\Phi .(k)=H \underset{\leftrightarrows}{\leftrightarrows} H^{2} H^{3} \underset{\Xi}{\leftrightarrows} \ldots
$$

Here and in the sequel, given a vector space $V$, we write $V^{n}=V \otimes \cdots \otimes V$, the $n$-fold tensor product. Notice that the chain complex associated with $\Phi .(k)$ is the (unnormalized) standard free resolution of the trivial left $H$-module $k$ (see [CE, Chap. X, Sect. 2], [We, 8.6.12, p. 283]), if we forget its right $K$-comodule structure.

Passing to the dual, first we have

Lemma 3.7. Let $Q$ be a left $H$-module. Regard $G(Q):=Q \otimes K$ as a right $K$ comodule via the right comultiplication by $K$ on the factor $K$, and as a left $H$ module via

$$
a(q \otimes f)=\sum a_{(1) H} q \otimes a_{(1) K}\left(a_{(2)} \rightarrow f\right)
$$

for $a \in H, q \otimes f \in Q \otimes K$. Then, $G(Q) \in \mathcal{C}$. Furthermore, $G$ defines a functor from the category $H$-Mod of left $H$-modules to $\mathcal{C}$, which is right adjoint to the forgetful functor $U: \mathcal{C} \rightarrow H$-Mod. 
Proof. It follows by $[\mathrm{S}$, Proposition 3.4] that $G(Q) \in \mathcal{C}$. For the last statement, we have an isomorphism

$$
\operatorname{Hom}_{H}(U(M), Q) \cong \operatorname{Hom}_{H}^{K}(M, G(Q))
$$

for $M \in \mathcal{C}, Q \in H$-Mod, which is given by $\phi \mapsto\left(m \mapsto \sum \phi\left(m_{(0)}\right) \otimes m_{(1)}\right)$.

Denote the composite $G U: \mathcal{C} \rightarrow \mathcal{C}$ simply by $G$. One sees again from We, 8.6.2] that $G$ forms a triple on $\mathcal{C}$, combined with the natural transformations $\eta: I \rightarrow G$, $\mu: G^{2} \rightarrow G$ defined by

$$
\begin{aligned}
& \eta_{M}: M \rightarrow M \otimes K, \quad \eta_{M}(m)=\sum m_{(0)} \otimes m_{(1)}, \\
& \mu_{M}: M \otimes K \otimes K \rightarrow M \otimes K, \quad \mu_{M}(m \otimes f \otimes g)=m \otimes \varepsilon(f) g
\end{aligned}
$$

for $M \in \mathcal{C}$. The triple $(G, \eta, \mu)$ determines a cosimplical object $\Psi \cdot(M):=G^{+1}(M)$ on $\mathcal{C}$ for each $M \in \mathcal{C}$. We need $\Psi \cdot(k)$, which looks like

$$
\Psi^{\cdot}(k)=K \rightleftarrows K^{2} \rightleftarrows K^{3} \rightleftarrows \cdots
$$

The associated cochain complex is the (unnormalized) standard cofree resolution of the trivial right $K$-comodule $k$, if we forget its left $H$-module structure.

One sees that, if $M, N \in \mathcal{C}$, the tensor product $M \otimes N$, given the diagonal $H$-action and the diagonal $K$-coaction, is an object in $\mathcal{C}$, and that the twisting $\tau: M \otimes N \rightarrow N \otimes M, \tau(m \otimes n)=n \otimes m$ is a morphism in $\mathcal{C}$. Thus, $\mathcal{C}=(\mathcal{C}, \otimes, k)$ forms a symmetric monoidal category, and hence commutative algebras or cocommutative coalgebras in $\mathcal{C}$ are well defined. For example, $K$ is a commutative algebra in $\mathcal{C}, H$ is a cocommutative coalgebra in $\mathcal{C}$, and $k$ is both. Furthermore, $F$ may be regarded as a functor from the category $\mathcal{C}_{c}$ of cocommutative coalgebras in $\mathcal{C}$ to itself. In fact, if $C \in \mathcal{C}_{c}$, then $F(C)=H \otimes C \in \mathcal{C}_{c}$, given the coalgebra structure of tensor product. Since $(F, \varepsilon, \delta)$ is seen to be a cotriple on $\mathcal{C}_{c}, \Phi .(k)$ is a simplical object in $\mathcal{C}_{c}$. Dually, $\Psi^{\cdot}(k)$ is a cosimplical object in the category $\mathcal{C}_{a}$ of commutative algebras in $\mathcal{C}$.

For $M, N \in \mathcal{C}$, we have a natural isomorphism of vector spaces

$$
\operatorname{Hom}_{H}^{K}(F(M), G(N)) \cong \operatorname{Hom}(M, N),
$$

which is obtained by composing the two familiar isomorphisms (see (3.6), (3.8)). If $M \in \mathcal{C}_{c}, N \in \mathcal{C}_{a}$, then this induces the isomorphism

$$
\operatorname{Reg}_{H}^{K}(F(M), G(N)) \cong \operatorname{Reg}(M, N)
$$

of abelian groups, where Reg (respectively, $\operatorname{Reg}_{H}^{K}$ ) indicates the linear (respectively, the $H$-linear and $K$-colinear) maps which are invertible under the convolution product.

Apply $\operatorname{Reg}_{H}^{K}($,$) to \Phi .(k)$ and $\Psi \cdot(k)$, and use the isomorphism (3.10). Then one obtains a double cosimplical object in the category of abelian groups, which looks 
like

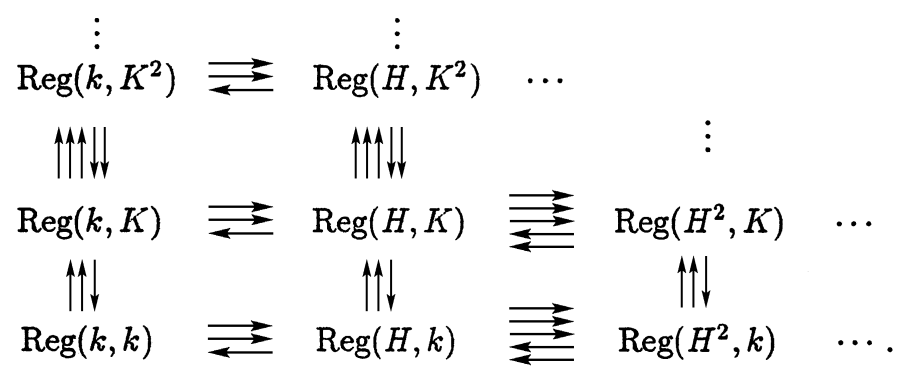

Consider the associated normalized double complex, whose $(p, q)$ th term, denoted by $\operatorname{Reg}_{+}\left(H^{p}, K^{q}\right)$, is the intersection of the kernels of the following $p+q$ codegeneracy operators $(i=1, \ldots, p ; j=1, \ldots, q)$ :

$$
\begin{array}{ll}
s_{i}: \operatorname{Reg}\left(H^{p}, K^{q}\right) \rightarrow \operatorname{Reg}\left(H^{p-1}, K^{q}\right), & s_{i} \phi=\phi\left(1^{i-1} \otimes \eta \otimes 1^{p-i}\right), \\
t_{j}: \operatorname{Reg}\left(H^{p}, K^{q}\right) \rightarrow \operatorname{Reg}\left(H^{p}, K^{q-1}\right), & t_{j} \phi=\left(1^{j-1} \otimes \varepsilon \otimes 1^{q-j}\right) \phi,
\end{array}
$$

where $\eta: k \rightarrow H$ is the unit of $H$. Furthermore, delete from the double complex just obtained its edge complexes (that is, the 0th row and the 0th column), and obtain

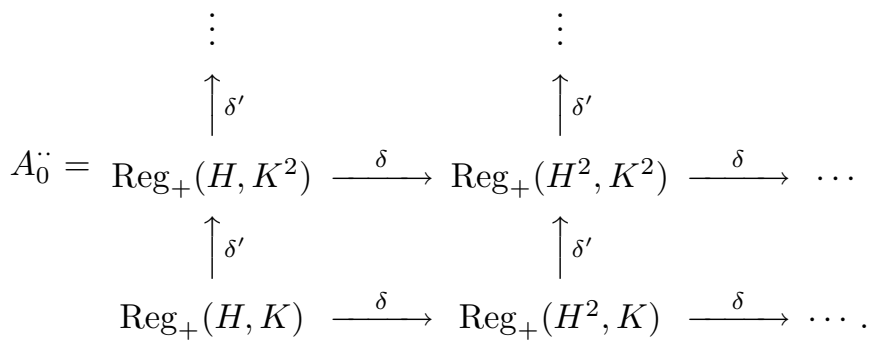

The differentials $\delta, \delta^{\prime}$ will be given explicitly just after Proposition 3.14.

Let $\operatorname{Aut}(K \# H)$ denote the group of the auto-equivalences of the split extension $(K \# H)$.

Proposition 3.11 ([Hf], Propositions 3.15, 6.5]). There are natural isomorphisms of abelian groups

$$
\begin{aligned}
& H^{0}\left(\operatorname{Tot} A_{0}^{\ddot{*}}\right) \cong \operatorname{Aut}(K \# H), \\
& H^{1}\left(\operatorname{Tot} A_{0}^{\ddot{*}}\right) \cong \operatorname{Opext}(H, K, \rightarrow, \rho) \text {. }
\end{aligned}
$$

Proof. The first isomorphism is given in the proof of [Hf, Proposition 6.5]. (Note that $H^{n}$ here means $H^{n+1}$ in $[\mathrm{Hf}]$.)

For later use, we describe the second isomorphism explicitly, following [Hf, Sect. $3]$. Let $(\sigma, \theta)$ be a 1-cocycle in $\operatorname{Tot} A_{0}$, where $\sigma \in \operatorname{Reg}_{+}\left(H^{2}, K\right), \theta \in \operatorname{Reg}_{+}\left(H, K^{2}\right)$. Define a bialgebra $K \#_{\sigma, \theta} H$ to be the vector space $K \otimes H$ equipped with the structures determined by

$$
\begin{aligned}
& (f \# a)(g \# b)=\sum f\left(a_{(1)}-g\right) \sigma\left(a_{(2)}, b_{(1)}\right) \# a_{(3)} b_{(2)}, \\
& \Delta(f \# a)=\sum\left(f_{(1)} a_{(1) \mathrm{I}} \# a_{(2) H}\right) \otimes\left(f_{(2)} a_{(1) \mathrm{II}} a_{(2) K} \# a_{(3)}\right), \\
& \text { unit }=1 \# 1, \quad \varepsilon(f \# a)=\varepsilon(f) \varepsilon(a)
\end{aligned}
$$


for $f \# a, g \# b \in K \#_{\sigma, \theta} H$, where $\theta a=\sum a_{\mathrm{I}} \otimes a_{\mathrm{II}}$. (If $\sigma, \theta$ are both trivial, that is, $\sigma=\varepsilon \otimes \varepsilon, \theta=\varepsilon$, then $K \#_{\sigma, \theta} H=K \# H$, the bismash product.) It is seen that $K \#_{\sigma, \theta} H$ has an antipode (see [Hf. p. 274, lines 17-18]), and it forms a cleft extension $\left(K \#_{\sigma, \theta} H\right)$ of $H$ by $K$, together with

$$
\begin{aligned}
\iota & =1 \otimes \eta: K=K \otimes k \rightarrow K \#_{\sigma, \theta} H, \\
\pi & =\varepsilon \otimes 1: K \#_{\sigma, \theta} H \rightarrow k \otimes H=H .
\end{aligned}
$$

The correspondence $(\sigma, \theta) \mapsto\left(K \#_{\sigma, \theta} H\right)$ induces the desired isomorphism.

Although the normalization Reg $\rightarrow$ Reg ${ }_{+}$brings no change on the total cohomology, we need the normalized $A_{0}^{\ddot{2}}$ for the sake of Proposition 3.14 below.

We will define two more double complexes, $B^{*}$ and $B_{0}$. Write

$$
H_{+}=\text {Coker } \eta, \quad K^{+}=\operatorname{Ker} \varepsilon,
$$

the cokernel of the unit of $H$ and the kernel of the counit of $K$, respectively. Construct the normalized chain complex associated with $\Phi .(k)$, which looks like the standard resolution of $k$, and delete from it the 0th term $H$. Then we have a chain complex in $\mathcal{C}$,

$$
X .(H)=0 \leftarrow H \otimes H_{+} \leftarrow H \otimes H_{+}^{2} \leftarrow H \otimes H_{+}^{3} \leftarrow \cdots,
$$

with each $H \otimes H_{+}^{p}$ given the quotient structure of $F^{p+1}(k)=H^{p+1}$. Notice that $X$. $(H)$ gives a resolution of $H^{+}=\operatorname{Ker} \varepsilon$ in $\mathcal{C}$, with the augmentation

$$
0 \leftarrow H^{+} \stackrel{\mu^{+}}{\leftarrow} H \otimes H_{+}, \quad a b-a \varepsilon(b) \leftarrow a \otimes \bar{b} .
$$

Dually, construct the normalized cochain complex associated with $\Psi \cdot(k)$, and delete from it the 0 th term $K$. Then we have a cochain complex in $\mathcal{C}$,

$$
Y^{\cdot}(K)=0 \rightarrow K^{+} \otimes K \rightarrow K^{+2} \otimes K \rightarrow K^{+3} \otimes K \rightarrow \cdots,
$$

which gives a resolution of $K_{+}=$Coker $\eta$ in $\mathcal{C}$, with

$$
0 \rightarrow K_{+} \stackrel{\Delta_{+}}{\rightarrow} K^{+} \otimes K, \quad \bar{f} \mapsto \Delta f-1 \otimes f .
$$

Applying $\operatorname{Hom}($,$) to X .(H)$ and $Y \cdot(K)$, we have

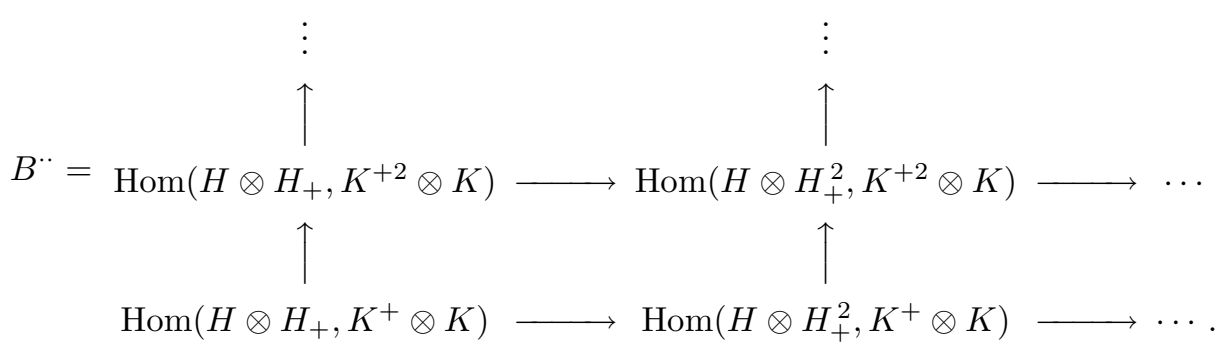

To make this into a double complex, we change the sign of the differentials in each even column, that is, the 0th column, the second column, and so forth (sign trick). Notice that the total complex $\operatorname{Tot} B^{\prime \prime}$ of $B^{\prime \prime}$ gives a resolution of $\operatorname{Hom}\left(H^{+}, K_{+}\right)$, with the augmentation

$$
0 \rightarrow \operatorname{Hom}\left(H^{+}, K_{+}\right) \rightarrow \operatorname{Hom}\left(H \otimes H_{+}, K^{+} \otimes K\right), \quad \phi \mapsto \Delta_{+} \phi \mu^{+} .
$$

(See the proof of Proposition 2.8.) 
Next, apply $\operatorname{Hom}_{H}^{K}(,$,$) to X .(H)$ and $Y^{\cdot}(K)$, and use the identification (3.9). Using the sign trick, we have a double complex of vector spaces

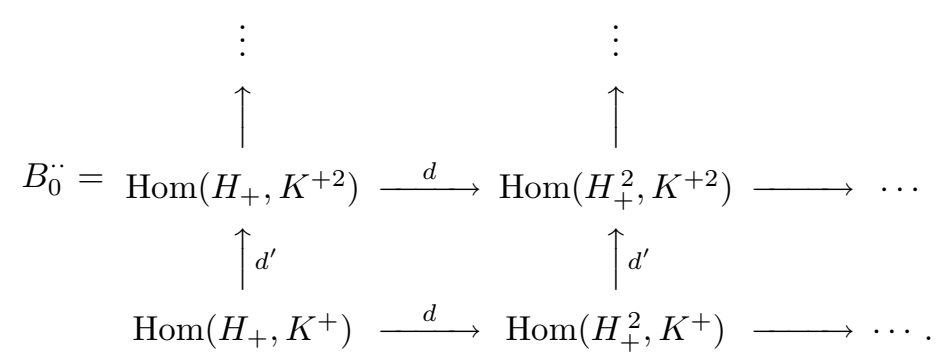

In the following proposition, we regard $B_{0}^{*}$ as a double complex of abelian groups, forgetting the scalar multiplication.

Proposition 3.14. Suppose $\operatorname{ch} k=0$. Suppose that $(H, K, \rightarrow, \rho)$ is a Singer pair of Hopf algebras with $H=U \mathfrak{f}$, the universal enveloping algebra of some Lie algebra $\mathfrak{f}$ (possibly of infinite dimension). Then there is a natural isomorphism

$$
A_{0}^{\ddot{*}} \cong B_{0}^{*}
$$

between the double complexes of abelian groups.

For the proof, we describe explicitly the differentials in $A_{0}^{*}$ and in $B_{0}^{*}$. Let $\phi \in \operatorname{Hom}\left(H^{p}, K^{q}\right)$. Define $d^{i}: \operatorname{Hom}\left(H^{p}, K^{q}\right) \rightarrow \operatorname{Hom}\left(H^{p+1}, K^{q}\right)(i=0,1, \ldots, p+1)$ by

$$
\begin{aligned}
& d^{0} \phi=\left(H \text {-action on } G^{q}(k)=K^{q}\right)(1 \otimes \phi), \\
& d^{i} \phi=\phi\left(1^{i-1} \otimes \mu \otimes 1^{p-i}\right) \quad(1 \leq i \leq p), \\
& d^{p+1} \phi=\phi \otimes \varepsilon
\end{aligned}
$$

with $\mu: H \otimes H \rightarrow H$ the product, and define $d^{j}: \operatorname{Hom}\left(H^{p}, K^{q}\right) \rightarrow \operatorname{Hom}\left(H^{p}, K^{q+1}\right)$ $(j=0,1, \ldots, q+1)$ by

$$
\begin{aligned}
& d^{\prime 0} \phi=(\phi \otimes 1)\left(K \text {-coaction on } F^{p}(k)=H^{p}\right), \\
& d^{\prime j} \phi=\left(1^{q-j} \otimes \Delta \otimes 1^{j-1}\right) \phi \quad(1 \leq j \leq q), \\
& d^{\prime q+1} \phi=\eta \otimes \phi
\end{aligned}
$$

with $\eta: k \rightarrow K$ the unit. The horizontal differentials $\delta$ and the vertical differentials $\delta^{\prime}$ in $A_{0}^{\ddot{*}}$ are given by

$$
\begin{aligned}
& \delta \phi=d^{0} \phi * d^{1} \phi^{-1} * \cdots * d^{p+1} \phi^{ \pm 1} \\
& \delta^{\prime} \phi=\left[d^{\prime 0} \phi * d^{\prime 1} \phi^{-1} * \cdots * d^{\prime q+1} \phi^{ \pm 1}\right]^{(-1)^{p}},
\end{aligned}
$$

where $\phi \in A_{0}^{p-1, q-1}=\operatorname{Reg}_{+}\left(H^{p}, K^{q}\right)$, while those $d, d^{\prime}$ in $B_{0}^{*}$ are given by

$$
\begin{aligned}
& d \phi=\sum_{i=0}^{p+1}(-1)^{i} d^{i} \phi, \\
& d^{\prime} \phi=(-1)^{p} \sum_{j=0}^{q+1}(-1)^{j} d^{\prime j} \phi,
\end{aligned}
$$

where $\phi \in B_{0}^{p-1, q-1}=\operatorname{Hom}\left(H_{+}^{p}, K^{+q}\right)$. 
Proof of Proposition 3.14. We modify the proof by Sweedler [Sw, Theorem 4.1].

Fix $p, q(>0)$, and write simply $A_{0}=A_{0}^{p-1, q-1}, B_{0}=B_{0}^{p-1, q-1}$. Since $K^{q}$ is in particular a left $H$-module algebra, it follows by [Sw p. 213, lines 8, 13-15] that, if $\phi \in B_{0}$ and $a \in H^{p}$, then $\phi^{n}(a)=0$ for large $n$, and hence that an element $\exp \phi$ in $\operatorname{Hom}\left(H^{p}, K^{q}\right)$ is well defined by

$$
\exp \phi(a)=e(a)+\sum_{n=1}^{\infty} \frac{\phi^{n}(a)}{n !} \quad\left(a \in H^{p}\right)
$$

where $e$ is the unit of $\operatorname{Hom}\left(H^{p}, K^{q}\right)$ and $\phi^{n}=\phi * \cdots * \phi$ ( $n$ times). One sees again by $\left[\mathrm{Sw}\right.$, p. 213, line 18] that $\exp \left(\phi+\phi^{\prime}\right)=\exp \phi * \exp \phi^{\prime}$ for $\phi, \phi^{\prime} \in B_{0}$. This implies that $\exp \phi$ is convolution-invertible, and further one sees that $\exp \phi \in A_{0}$. Thus we have a group map exp : $B_{0} \rightarrow A_{0}$. We claim that this is an isomorphism. In fact, since $\phi \in A_{0}$ implies $\phi-e \in B_{0}$, the map

$$
\log \phi=\sum_{n=1}^{\infty} \frac{(-1)^{n-1}}{n}(\phi-e)^{n}
$$

is well defined and is contained in $B_{0}$. It is standard to see that the map log: $A_{0} \rightarrow$ $B_{0}$ thus obtained is an inverse of exp.

It remains to show that exp is compatible with the differentials. Let $D$ be any one of $d^{i}, d^{\prime j}$ defined above. Then, if $\phi \in B_{0}^{p-1, q-1}$, then $D \phi \in B_{0}^{p, q-1}$ or $\in B_{0}^{p-1, q}$, and so $\exp D \phi$ is well defined. Since $D$ is an algebra map, we have $D\left(\phi^{n}\right)=(D \phi)^{n}$, and hence $D(\exp \phi)=\exp D \phi$, which yields

$$
\delta(\exp \phi)=\exp d \phi, \quad \delta^{\prime}(\exp \phi)=\exp d^{\prime} \phi,
$$

as desired.

\section{From Hopf Algebra Extensions to Lie Bialgebra Extensions}

Let $(H, J, \triangleright, \triangleleft)$ be a matched pair of cocommutative Hopf algebras (Definition 2.1). Accordingly, one forms the Hopf algebra $H \bowtie J$. Denote by $H \bowtie J$-Mod the category of left $H \bowtie J$-modules. Define $K=J^{\circ}$, the dual Hopf algebra of $J$ (see [Mo, Theorem 9.1.3]), which consists of elements in $J^{*}$ vanishing on some cofinite ideal of $J$ (a cofinite ideal is an ideal whose codimension $\operatorname{dim} J / I$ is finite). Thus, $K$ is a commutative Hopf algebra. Suppose that $H$ is a locally finite left $J$-module under $\triangleright$, which means that $H$ is a (directed) union of finite-dimensional $J$-submodules. Then it follows by [Mo, Lemma 1.6.4 2)] that there is a unique right $K$-comodule structure $\rho: H \rightarrow H \otimes K, \rho a=\sum a_{H} \otimes a_{K}$, on $H$ such that $x \triangleright a=\sum a_{H}\left\langle x, a_{K}\right\rangle$ for $x \in J, a \in H$.

Lemma 4.1. The transpose $\rightarrow: H \otimes J^{*} \rightarrow J^{*}$ of $\triangleleft$ stabilizes $K\left(\subset J^{*}\right)$. Denote the induced action by $\rightarrow: H \otimes K \rightarrow K$, too. Then, $(H, K, \rightarrow, \rho)$ forms a Singer pair of Hopf algebras, and the category $\mathcal{C}(H, K, \rightarrow, \rho)$ defined in Definition 3.4 is the full subcategory of $H \bowtie J$-Mod consisting of the objects which are locally finite $J$-modules (provided the $H \bowtie J$-action is restricted to $J=k \otimes J$ ). 
Proof. Let $a \in H, x, y \in J, f \in K$. Then we have

$$
\begin{aligned}
\langle x y, a & \rightarrow f\rangle=\langle x y \triangleleft a, f\rangle \\
& =\sum\left\langle\left(x \triangleleft\left(y_{(1)} \triangleright a_{(1)}\right)\right)\left(y_{(2)} \triangleleft a_{(2)}\right), f\right\rangle \\
& =\sum\left\langle\left(x \triangleleft a_{(1) H}\right)\left(y_{(2)} \triangleleft a_{(2)}\right), f\right\rangle\left\langle y_{(1)}, a_{(1) K}\right\rangle \\
& =\sum\left\langle x \triangleleft a_{(1) H}, f_{(1)}\right\rangle\left\langle y_{(2)} \triangleleft a_{(2)}, f_{(2)}\right\rangle\left\langle y_{(1)}, a_{(1) K}\right\rangle \\
& =\sum\left\langle x, a_{(1) H} \rightarrow f_{(1)}\right\rangle\left\langle y, a_{(1) K}\left(a_{(2)} \rightarrow f_{(2)}\right)\right\rangle .
\end{aligned}
$$

This implies by [Mo, Lemma 9.1.1] that $a \rightarrow f \in K$, and that the equation (3.3.2) holds. From the fact that $J$ is a right $H$-module coalgebra under $\triangleleft$, one deduces easily that $K$ is a left $H$-module algebra under $\rightarrow$. Similarly, from the fact that $H$ is a left $J$-module coalgebra under $\triangleright$, one deduces that $H$ is a right $K$-comodule coalgebra under $\rho$. Furthermore, the equation (3.3.1) follows from the equation (2.1.1), so we conclude that $(H, K, \rightarrow, \rho)$ is a Singer pair of Hopf algebras.

Again by [Mo, Lemma 1.6.4 2)], a right $K$-comodule $M$ is none other than a locally finite left $J$-module, so that the category of right $K$-comodules is a full subcategory of the category of left $J$-modules. Suppose that $M$ is also a left $H$ module. Then one sees that the equation (2.2) holds if and only if the equation given in Definition 3.4 holds. This yields the last statement.

Corollary 4.2. Let $H, J$ be cocommutative Hopf algebras, and define $K=J^{\circ}$. There is a natural correspondence, as given above, from the set of the structures $(\triangleright, \triangleleft)$ of a matched pair on $(H, J)$ such that $\triangleright$ makes $H$ a locally finite left $J$-module, to the set of the structures $(-, \rho)$ of a Singer pair on $(H, K)$. The correspondence is injective if $J$ is proper (or residually finite-dimensional) in the sense of $\mathrm{MO}$, Definition 9.2.9].

Proof. It remains to show the last statment. By [Mo Lemma 1.6.4 2)], $\triangleright$ is recovered from $\rho$. If $J$ is proper, then by definition the natural algebra map $J \rightarrow\left(J^{\circ}\right)^{*}=K^{*}$ is an injection. Through this injection, restrict the transpose of $\rightarrow$ onto $J$. Then, $\triangleleft$ is recovered.

In the remainder of this section, we suppose $\operatorname{ch} k=0$, and let $\mathfrak{f}, \mathfrak{g}$ be finitedimensional Lie algebras. Since $U \mathfrak{g}$ is proper (see [Mo, p. 157]), we obtain the first part of the following proposition by putting Propositions 1.6, 2.4 and Corollary 4.2 together. (Notice that, since every matched pair $(U \mathfrak{f}, U \mathfrak{g}, \triangleright, \triangleleft)$ of Hopf algebras is induced from such a pair $(\mathfrak{f}, \mathfrak{g}, \triangleright, \triangleleft)$ of Lie algebras, $U \mathfrak{f}$ is a locally finite left $U \mathfrak{g}$-module under $\triangleright$, and hence that Corollary 4.2 can apply.)

Proposition 4.3. There is a natural injection from the set of the structures $(\rightarrow, \rho)$ of a Singer pair of Lie bialgebras on $\left(\mathfrak{f}, \mathfrak{g}^{*}\right)$ to the set of the structures $\left(-^{\prime}, \rho^{\prime}\right)$ of a Singer pair of Hopf algebras on $\left(U \mathfrak{f}, U \mathfrak{g}^{\circ}\right)$. This is a bijection if $\mathfrak{g}=[\mathfrak{g}, \mathfrak{g}]$ (which holds, for example, if $\mathfrak{g}$ is semisimple).

Proof. Write

$$
H=U \mathfrak{f}, \quad J=U \mathfrak{g}, \quad K=J^{\circ}=U \mathfrak{g}^{\circ} .
$$

Dualizing the natural inclusion $\mathfrak{g} \hookrightarrow U \mathfrak{g}$, we have the restriction map $\omega: K=$ $U \mathfrak{g}^{\circ} \rightarrow \mathfrak{g}^{*}$, which is a surjection by the Ado theorem B, Chap. I, Sect. 7, Theorem $2]$. 
To show the last statement, it suffices to prove that the correspondence given in Corollary 4.2 is surjective. Suppose that a structure $(\rightarrow, \rho)$ of a Singer pair on $(H, K)$ is given. One sees that $\rho$ comes from some action $\triangleright: J \otimes H \rightarrow H$ that makes $H$ a (locally finite) left $J$-module coalgebra. Let $\triangleleft: K^{*} \otimes H \rightarrow K^{*}$ be the transpose of $\rightarrow$. We will show that this action stabilizes $J$, which is regarded by properness as a subalgebra of $K^{*}$. It is shown in [T, Lemma 1.2] that $\varepsilon(a \rightarrow f)=\varepsilon(a) \varepsilon(f)$ for $a \in H, f \in K$. This implies that the module-algebra action $\rightarrow$ stabilizes the ideals $K^{+} \supset\left(K^{+}\right)^{2}$, so that an $H$-action on $K^{+} /\left(K^{+}\right)^{2}$ is induced.

It is shown by Hochschild [ $\underline{\mathrm{H} 2}$, Theorem 6.1] that $\mathfrak{g}=[\mathfrak{g}, \mathfrak{g}]$ if and only if $\mathfrak{g}=$ $P\left(K^{\circ}\right)$, the primitive elements in $K^{\circ}$. The last equality holds if and only if $\left.\omega\right|_{K^{+}}$: $K^{+} \rightarrow \mathfrak{g}^{*}$ induces an isomorphism $K^{+} /\left(K^{+}\right)^{2} \cong \mathfrak{g}^{*}$, since $P\left(K^{\circ}\right) \cong\left(K^{+} /\left(K^{+}\right)^{2}\right)^{*}$ (see, for example, [Wa, Sect. 11.3]). Suppose $\mathfrak{g}=[\mathfrak{g}, \mathfrak{g}]$. Then the preceding result shows that $\rightarrow$ induces an action $H \otimes \mathfrak{g}^{*} \rightarrow \mathfrak{g}^{*}$, whose transpose $\mathfrak{g} \otimes H \rightarrow \mathfrak{g}$ coincides with the restriction of $\triangleleft$, as is easily seen. In particular, one sees that $\triangleleft$ stabilizes $\mathfrak{g}$. Hence we see from (3.3.2) that, if $a \in H, x \in J, y \in \mathfrak{g}$, then

$$
x y \triangleleft a=\sum\left(x \triangleleft\left(y_{(1)} \triangleright a_{(1)}\right)\right)\left(y_{(2)} \triangleleft a_{(2)}\right)
$$

in $K^{*}$. Use induction on the length in the expression of $x$ as a product of elements in $\mathfrak{g}$. Then the last equation yields $J \triangleleft H \subset J$, as desired. Denote the induced action by $\triangleleft: J \otimes H \rightarrow J$, too. Using the properness of $J$, one sees that $(H, J, \triangleright, \triangleleft)$ is a matched pair. Clearly, $(\triangleright, \triangleleft)$ corresponds to $(\rightarrow, \rho)$.

Remark 4.5. Suppose $(\rightarrow, \rho) \mapsto\left(\rightarrow^{\prime}, \rho^{\prime}\right)$ via the correspondence just obtained. Then it is easily seen that $(\rightarrow, \rho)$ is recovered from $\left(-^{\prime}, \rho^{\prime}\right)$ as a unique pair of maps which make the following diagrams commute:

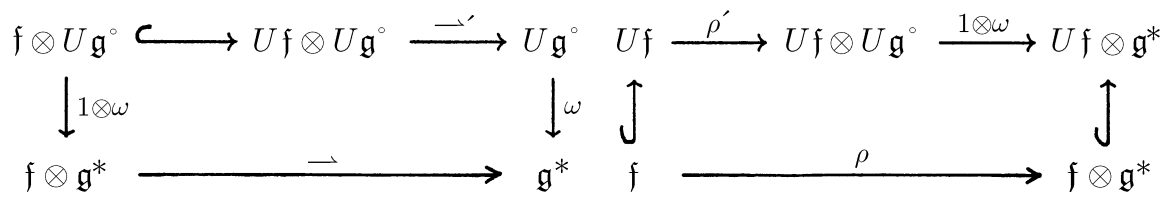

The following example shows that the correspondence in Proposition 4.3 is not necessarily surjective unless $\mathfrak{g}=[\mathfrak{g}, \mathfrak{g}]$.

Example 4.6. Suppose that $k$ is an algebraically closed field (of characteristic zero). Let $\mathfrak{f}=k a, \mathfrak{g}=k x$ be as in Example 1.2. Then, $U \mathfrak{f}=k[a], U \mathfrak{g}=k[x]$. Define $f \in U \mathfrak{g}^{*}$ by $\left\langle f, x^{n}\right\rangle=\delta_{1, n}$, where $0 \leq n \in \mathbb{Z}$. Then, $f$ is a primitive element in $U \mathfrak{g}^{\circ}$. For each $c \in k$, define an algebra map $g_{c}: U \mathfrak{g}=k[x] \rightarrow k$ by $g_{c}(x)=c$. Then, $g_{c}$ is a group-like element in $U \mathfrak{g}^{\circ}$, and $g_{c} * g_{c^{\prime}}=g_{c+c^{\prime}}\left(c, c^{\prime} \in k\right)$. Moreover, by [Mo, Example 9.1.7], $U \mathfrak{g}^{\circ}=k[f] \otimes k \Gamma$, the tensor product of the polynimial algebra $k[f]$ and the group algebra $k \Gamma$ spanned by $\Gamma=\left\{g_{c}: c \in k\right\}$. Define a module action $\rightarrow: U \mathfrak{f} \otimes U \mathfrak{g}^{\circ} \rightarrow U \mathfrak{g}^{\circ}$ by

$$
a \rightarrow f^{n} g_{c}=c f^{n+1} g_{c} \quad(0 \leq n \in \mathbb{Z}, c \in k) .
$$

Then one sees easily that $\rightarrow$ makes $U \mathfrak{g}^{\circ}$ into a left $U \mathfrak{f}$-module algebra and coalgebra, so that $\rightarrow$, together with the trivial coaction $\rho: U \mathfrak{f} \rightarrow U \mathfrak{f} \otimes U \mathfrak{g}^{\circ}, \rho a^{m}=a^{m} \otimes 1$, gives to $\left(U \mathfrak{f}, U \mathfrak{g}^{\circ}\right)$ a structure of a Singer pair of Hopf algebras. But, $(\rightarrow, \rho)$ cannot be obtained from any structure of a Singer pair of Lie bialgebras on $\left(\mathfrak{f}, \mathfrak{g}^{*}\right)$. To see this, it suffices to show that the action $\rightarrow$ cannot factor through $\omega: U \mathfrak{g}^{\circ} \rightarrow \mathfrak{g}^{*}$. In 
fact, for $0 \neq c \in k$, we have $\omega(c f)=c \omega(f)=\omega\left(g_{c}\right)$, but $\omega(a \rightarrow c f)=0 \neq c \omega(f)=$ $\omega\left(a \rightarrow g_{c}\right)$.

Suppose $(\rightarrow, \rho) \mapsto\left(\rightarrow^{\prime}, \rho^{\prime}\right)$ via the correspondence in Proposition 4.3. By virtue of the injectivity of the correspondence, we may write $\left(-^{\prime}, \rho^{\prime}\right)=(\rightarrow, \rho)$, and say that a Singer pair $\left(\mathfrak{f}, \mathfrak{g}^{*}, \rightarrow, \rho\right)$ of Lie bialgebras induces a Singer pair $\left(U \mathfrak{f}, U \mathfrak{g}^{\circ}, \rightarrow, \rho\right)$ of Hopf algebras. Correspondingly, a matched pair $(\mathfrak{f}, \mathfrak{g}, \triangleright, \triangleleft)$ of Lie algebras and a matched pair $(U \mathfrak{f}, U \mathfrak{g}, \triangleright, \triangleleft)$ of Hopf algebras arise by Propositions 1.6 and 2.4, respectively.

In the following, we use the notation (4.4) again. Recall the chain complex $V$.(f) of left $H \bowtie J$-modules defined in Section 2. Delete from $V$.(f) the 0th term $V_{0}(\mathfrak{f})=H$, to obtain

$$
X .(\mathfrak{f})=0 \leftarrow H \otimes \mathfrak{f} \leftarrow H \otimes \wedge^{2} \mathfrak{f} \leftarrow H \otimes \wedge^{3} \mathfrak{f} \leftarrow \cdots .
$$

This is a resolution of $H^{+}$in $H \bowtie J$-Mod, given the augmentation

$$
0 \leftarrow H^{+} \stackrel{\nu^{+}}{\leftarrow} H \otimes \mathfrak{f}, \quad a b \longleftarrow a \otimes b .
$$

Recall the chain complex $X$. $(H)$ defined in Section 3, which may be regarded by Lemma 4.1 as a chain complex in $H \bowtie J$-Mod. This is a resolution of $H^{+}$, too. Write $u\left\{a_{1}, \ldots, a_{p}\right\}$ for an element $u \otimes \overline{a_{1}} \ldots \otimes \overline{a_{p}}$ in $H \otimes H_{+}^{p}$, where $\bar{a}$ denotes the natural image of $a(\in H)$ in $H_{+}$. For each $p=1,2, \ldots$, we define a linear map

$$
\varphi=\varphi_{p-1}: X_{p-1}(\mathfrak{f})=H \otimes \wedge^{p} \mathfrak{f} \rightarrow H \otimes H_{+}^{p}=X_{p-1}(H)
$$

by

$$
\varphi\left(u\left\langle a_{1}, \ldots, a_{p}\right\rangle\right)=\sum_{\sigma \in \mathfrak{S}_{p}}(\operatorname{sgn} \sigma) u\left\{a_{\sigma(1)}, \ldots, a_{\sigma(p)}\right\} .
$$

By [CE, Chap. XIII, Sect.7], $\varphi_{p}(p=0,1, \ldots)$ are all $H$-linear injections, and are compatible with the differentials. Moreover, we claim:

\section{Lemma 4.8.}

$$
\varphi .: X .(\mathfrak{f}) \rightarrow X .(H)
$$

is a map of chain complexes in $H \bowtie J$-Mod.

Proof. It remains to show that each $\varphi=\varphi_{p-1}$ is $J$-linear.

Notice that, if $M \in \mathcal{C}$, then the $J$-action on $F(M)=H \otimes M$ is the same as the one given in Proposition 2.31 ). Thus the $J$-action on $H \otimes H_{+}^{p}$ is given by

$$
\begin{aligned}
& x\left(u\left\{a_{1}, \ldots, a_{p}\right\}\right) \\
& \quad=\sum(x \triangleright u)\left\{(x \triangleleft u) \triangleright a_{1},\left(x \triangleleft u a_{1}\right) \triangleright a_{2}, \ldots,\left(x \triangleleft u a_{1} \ldots a_{p-1}\right) \triangleright a_{p}\right\}
\end{aligned}
$$

for $x \in J$, where the abbreviated sigma notation is used and the sum is taken over $(x),(u),\left(a_{1}\right), \ldots,\left(a_{p-1}\right)$. One observes that, if $u=1$ and $a_{1}, \ldots, a_{p} \in \mathfrak{f}$, then the right-hand side of the equation equals

$$
\sum_{(x)}\left\{x_{(1)} \triangleright a_{1}, x_{(2)} \triangleright a_{2}, \ldots, x_{(p)} \triangleright a_{p}\right\},
$$

since $\left\{a_{1}, a_{2}, \ldots, a_{p}\right\}=0$ if either $a_{1}, a_{2}, \ldots$ or $a_{p}=1$. 
Let $x \in \mathfrak{g}, a_{i} \in \mathfrak{f}(i=1, \ldots, p)$. Then we have

$$
\begin{aligned}
\varphi & \left(x\left\langle a_{1}, \ldots, a_{p}\right\rangle\right)=\sum_{i} \varphi\left\langle a_{1}, \ldots, x \triangleright a_{i}, \ldots, a_{p}\right\rangle \\
& \left.=\sum_{i} \sum_{\sigma} \operatorname{sgn} \sigma\left\{a_{\sigma(1)}, \ldots, x \triangleright a_{\sigma(j)}, \ldots, a_{\sigma(p)}\right\} \quad \text { (where } j=\sigma^{-1}(i)\right) \\
& =\sum_{\sigma} \operatorname{sgn} \sigma \sum_{j}\left\{a_{\sigma(1)}, \ldots, x \triangleright a_{\sigma(j)}, \ldots, a_{\sigma(p)}\right\} \\
& =\sum_{\sigma}(\operatorname{sgn} \sigma) x\left\{a_{\sigma(1)}, \ldots, a_{\sigma(p)}\right\}=x \varphi\left\langle a_{1}, \ldots, a_{p}\right\rangle .
\end{aligned}
$$

For the fourth equality, the preceding observation was applied. It is now easy to see that $\varphi\left(x\left(u\left\langle a_{1}, \ldots, a_{p}\right\rangle\right)\right)=x \varphi\left(u\left\langle a_{1}, \ldots, a_{p}\right\rangle\right)$ for $u \in H$, and hence that $\varphi$ is $J$-linear.

Recall the cochain complex $Y^{\cdot}(K)$ defined in Section 3, which may be regarded again by Lemma 4.1 as a resolution of $K_{+}$in $H \bowtie J$-Mod. We apply to $J$ the procedure of constructing $X$. $(H)$, using a mirror, and then obtain the chain complex

$$
X_{.}^{\prime}(J)=0 \leftarrow J_{+} \otimes J \leftarrow J_{+}^{2} \otimes J \leftarrow J_{+}^{3} \otimes J \leftarrow \cdots
$$

of right $H \bowtie J$-modules, the dual of which is the cochain complex

$$
X^{\prime} \cdot(J)^{*}=0 \rightarrow\left(J_{+} \otimes J\right)^{*} \rightarrow\left(J_{+}^{2} \otimes J\right)^{*} \rightarrow\left(J_{+}^{3} \otimes J\right)^{*} \rightarrow \cdots
$$

of left $H \bowtie J$-modules. The inclusion $K \hookrightarrow J^{*}$ naturally induces left $H \bowtie J$-linear injections

$$
\left(K^{+}\right)^{q} \otimes K \rightarrow\left(J_{+}^{*}\right)^{q} \otimes J^{*} \hookrightarrow\left(J_{+}^{q} \otimes J\right)^{*} \quad(q=1,2, \ldots),
$$

which further give an injection $Y^{\cdot}(K) \rightarrow X^{\prime}(J)^{*}$ between the cochain complexes. This allows us to regard $Y^{\cdot}(K)$ as a subcomplex of $X^{\prime}(J)^{*}$.

Lemma 4.9. Let $Q, Q^{\prime}$ be finite-dimensional right $H$-modules. Then, $Q^{*}, Q^{\prime *}$ are left $H$-modules with the transposed action.

1) Regard the object $G\left(Q^{*}\right)=Q^{*} \otimes K$ in the category $\mathcal{C}$ as a left $H \bowtie J$-module by Lemma 4.1. Regard $Q \otimes J$ as a right $H \bowtie J$-module by Proposition 2.3 2), and then regard $(Q \otimes J)^{*}$ as a left $H \bowtie J$-module. Then the natural injection $Q^{*} \otimes K \rightarrow Q^{*} \otimes J^{*}=(Q \otimes J)^{*}$ is $H \bowtie J$-linear, through which we regard $Q^{*} \otimes K$ as an $H \bowtie J$-submodule of $(Q \otimes J)^{*}$.

2) A right $H \bowtie J$-linear map $Q \otimes J \rightarrow Q^{\prime} \otimes J$ induces a left $H \bowtie J$-linear map

$$
Q^{\prime *} \otimes K=\operatorname{Hom}_{J}\left(Q^{\prime} \otimes J, K\right) \rightarrow \operatorname{Hom}_{J}(Q \otimes J, K)=Q^{*} \otimes K
$$

through the canonical isomorphisms $V^{*} \otimes K=\operatorname{Hom}_{J}(V \otimes J, K)$ for $V=Q, Q^{\prime}$. (In $\operatorname{Hom}_{J}(, K), K$ is regarded as a right $J$-module in a natural way.)

Proof. 1) This is seen directly.

2) A right $H \bowtie J$-linear map $Q \otimes J \rightarrow Q^{\prime} \otimes J$ induces a left $H \bowtie J$-linear map

$$
\left(Q^{\prime} \otimes J\right)^{*}=\operatorname{Hom}_{J}\left(Q^{\prime} \otimes J, J^{*}\right) \rightarrow \operatorname{Hom}_{J}\left(Q \otimes J, J^{*}\right)=(Q \otimes J)^{*},
$$

which extends the linear map described above. Hence it should be $H \bowtie J$-linear by Part 1. 
Recall the chain complex $V^{\prime}(\mathfrak{g})$ of right $H \bowtie J$-modules defined in Section 2 . Delete from $V^{\prime}(\mathfrak{g})$ the 0 th term $V_{0}^{\prime}(\mathfrak{g})=J$, to obtain

$$
X^{\prime}(\mathfrak{g})=0 \leftarrow \mathfrak{g} \otimes J \leftarrow \wedge^{2} \mathfrak{g} \otimes J \leftarrow \wedge^{3} \mathfrak{g} \otimes J \leftarrow \cdots,
$$

the dual of which is the cochain complex

$$
X^{\prime}(\mathfrak{g})^{*}=0 \rightarrow(\mathfrak{g} \otimes J)^{*} \rightarrow\left(\wedge^{2} \mathfrak{g} \otimes J\right)^{*} \rightarrow\left(\wedge^{3} \mathfrak{g} \otimes J\right)^{*} \rightarrow \cdots
$$

in $H \bowtie J$-Mod. Consider the standard complex

$$
0 \rightarrow \operatorname{Hom}(k, K) \rightarrow \operatorname{Hom}(\mathfrak{g}, K) \rightarrow \operatorname{Hom}\left(\wedge^{2} \mathfrak{g}, K\right) \rightarrow \cdots
$$

for computing the cohomology group $H^{\cdot}(\mathfrak{g}, K)$ of $\mathfrak{g}$ with coefficients $K=U \mathfrak{g}^{\circ}$, the natural right $\mathfrak{g}$-Lie module. By deleting from this cochain complex its 0th term $\operatorname{Hom}(k, K)$, we obtain

$$
Y^{\cdot}\left(\mathfrak{g}^{*}\right)=0 \rightarrow \operatorname{Hom}(\mathfrak{g}, K) \rightarrow \operatorname{Hom}\left(\wedge^{2} \mathfrak{g}, K\right) \rightarrow \operatorname{Hom}\left(\wedge^{3} \mathfrak{g}, K\right) \rightarrow \cdots
$$

Since $\operatorname{Hom}\left(\wedge^{q} \mathfrak{g}, K\right)=\left(\wedge^{q} \mathfrak{g}\right)^{*} \otimes K$, it follows by Lemma 4.9 that $Y^{\cdot}\left(\mathfrak{g}^{*}\right)$ is a cochain complex in $H \bowtie J$-Mod and that we may regard it as a subcomplex of $X^{\prime}(\mathfrak{g})^{*}$. Define a map $\varphi^{\prime}$ : $X^{\prime}$. $(\mathfrak{g}) \rightarrow X^{\prime}(J)$ of chain complexes analogously to the definition of $\varphi$. (see Lemma 4.8). If the dual map $X^{\prime}(J)^{*} \rightarrow X^{\prime}(\mathfrak{g})^{*}$ of $\varphi^{\prime}$. is restricted onto $Y^{\cdot}(K)$, then we have a map

$$
\psi^{\cdot}: Y^{\cdot}(K) \rightarrow Y^{\cdot}\left(\mathfrak{g}^{*}\right)
$$

of cochain complexes in $H \bowtie J$-Mod, since each $\operatorname{Hom}\left(\wedge^{q} \mathfrak{g}, K\right)$ is the largest locally finite $J$-submodule of $\left(\wedge^{q} \mathfrak{g} \otimes J\right)^{*}$.

Apply $\operatorname{Hom}($,$) to X .(\mathfrak{f})$ and $Y^{\cdot}\left(\mathfrak{g}^{*}\right)$, and obtain the double complex

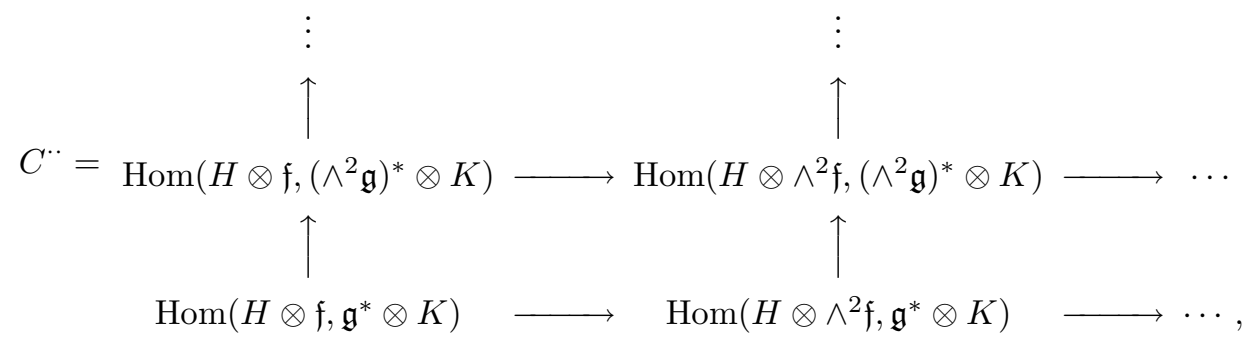

where the sign of the differentials in each even column is changed. From $\varphi$. and $\psi$, we define a map of double complexes by

$$
\alpha^{*}=\operatorname{Hom}\left(\varphi ., \psi^{*}\right): B^{*} \rightarrow C^{\cdot \cdot} .
$$

Let $M, N$ be left $H \bowtie J$-modules. Let $M_{0}$ denote the $H \bowtie J$-invariants of $M$, that is, the largest $H \bowtie J$-submodule of $M$ on which $H \bowtie J$ acts trivially. $\operatorname{Regard} \operatorname{Hom}(M, N)$ as a left $H \bowtie J$-module via the conjugate action. Then we have $\operatorname{Hom}(M, N)_{0}=\operatorname{Hom}_{H \bowtie J}(M, N)$. We see that $B^{*}$ and $C^{\cdot}$ are both double complexes in $H \bowtie J$-Mod, and that their $H \bowtie J$-invariants are $B_{0}^{*}$ and $C_{0}^{\cdot}$, respectively: $\left(B^{*}\right)_{0}=B_{0}^{*},\left(C^{\cdot \cdot}\right)_{0}=C_{0}^{\cdot .}$. Since $\alpha^{*}$ consists of $H \bowtie J$-linear maps, it induces a map of double complexes

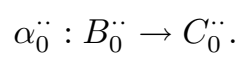

It will be proved in Section 6 that $\alpha_{0}^{\ddot{~}}$ induces linear isomorphisms

$$
H^{n}\left(\operatorname{Tot} B_{0}^{\cdot \cdot}\right) \cong H^{n}\left(\operatorname{Tot} C_{0}^{\cdot \cdot}\right) \quad(n=0,1) .
$$

Combining this with Propositions 2.9, 3.11 and 3.14, we claim the following. 
Theorem 4.11. Suppose $\operatorname{ch} k=0$. Suppose that a Singer pair $\left(\mathfrak{f}, \mathfrak{g}^{*}, \rightarrow, \rho\right)$ of Lie bialgebras induces a Singer pair $\left(U \mathfrak{f}, U \mathfrak{g}^{\circ}, \rightarrow, \rho\right)$ of Hopf algebras. Then there are natural isomorphisms of groups

$$
\begin{aligned}
& \kappa_{0}: \operatorname{Aut}\left(U \mathfrak{g}^{\circ} \# U \mathfrak{f}\right) \cong \operatorname{Aut}\left(\mathfrak{g}^{*} \bowtie \mathfrak{f}\right), \\
& \kappa_{1}: \operatorname{Opext}\left(U \mathfrak{f}, U \mathfrak{g}^{\circ}, \rightarrow, \rho\right) \cong \operatorname{Opext}\left(\mathfrak{f}, \mathfrak{g}^{*}, \rightarrow, \rho\right) .
\end{aligned}
$$

This implies that each of the groups $\operatorname{Aut}\left(U \mathfrak{g}^{\circ} \# U \mathfrak{f}\right), \operatorname{Opext}\left(U \mathfrak{f}, U \mathfrak{g}^{\circ}, \rightarrow, \rho\right)$ is either trivial or infinite, since it is, by Proposition 2.9, in 1-1 correspondence with a vector space over the infinite field $k$.

The theorem, combined with Theorem 2.10, yields the following.

Corollary 4.12. Let $\left(\mathfrak{f}, \mathfrak{g}^{*}, \rightarrow, \rho\right),\left(U \mathfrak{f}, U \mathfrak{g}^{\circ}, \rightarrow, \rho\right)$ be as above. (Then one can form the Lie algebra $\mathfrak{f} \bowtie \mathfrak{g}$ as in Theorem 2.10.) We have an exact sequence

$$
\begin{aligned}
0 & \rightarrow H^{1}(\mathfrak{f} \bowtie \mathfrak{g}, k) \rightarrow H^{1}(\mathfrak{f}, k) \oplus H^{1}(\mathfrak{g}, k) \rightarrow \operatorname{Aut}\left(U \mathfrak{g}^{\circ} \# U \mathfrak{f}\right) \\
& \rightarrow H^{2}(\mathfrak{f} \bowtie \mathfrak{g}, k) \rightarrow H^{2}(\mathfrak{f}, k) \oplus H^{2}(\mathfrak{g}, k) \rightarrow \operatorname{Opext}\left(U \mathfrak{f}, U \mathfrak{g}^{\circ}, \rightarrow, \rho\right) \\
& \rightarrow H^{3}(\mathfrak{f} \bowtie \mathfrak{g}, k) \rightarrow H^{3}(\mathfrak{f}, k) \oplus H^{3}(\mathfrak{g}, k) .
\end{aligned}
$$

Corollary 4.13. Let $\left(U \mathfrak{f}, U \mathfrak{g}^{\circ}, \rightarrow, \rho\right)$ be a Singer pair of Hopf algebras. If either 1) $\mathfrak{f}$ is semisimple and $\rightarrow$ is trivial, or 2) $\mathfrak{g}$ is semisimple and $\rho$ is trivial, then the groups $\operatorname{Aut}\left(U \mathfrak{g}^{\circ} \# U \mathfrak{f}\right)$ and $\operatorname{Opext}\left(U \mathfrak{f}, U \mathfrak{g}^{\circ}\right)$ are both trivial.

Proof. Notice that, under 1) or 2), the given Singer pair of Hopf algebras is induced from a Singer pair of Lie bialgebras (see the proof of Proposition 4.13). Then the conclusion follows from Corollary 2.11 and Theorem 4.11.

Combine Theorem 4.11 with the last statement of Proposition 4.3. Then we get the next theorem by taking the union of the isomorphisms $\kappa_{1}$ ranging over all $(\rightarrow, \rho)$.

Theorem 4.14. Suppose $\operatorname{ch} k=0$ and $\mathfrak{g}=[\mathfrak{g}, \mathfrak{g}]$. Then there is a natural bijection

$$
\operatorname{Ext}\left(U \mathfrak{f}, U \mathfrak{g}^{\circ}\right) \cong \operatorname{Ext}\left(\mathfrak{f}, \mathfrak{g}^{*}\right) .
$$

Remark 4.15. Suppose that $k$ is an algebraically closed field of characteristic zero. It follows from [H2, Sect. 3] that a commutative Hopf algebra is in the form $U \mathfrak{g}^{\circ}$, where $\mathfrak{g}$ is a finite-dimensional Lie algebra with $\mathfrak{g}=[\mathfrak{g}, \mathfrak{g}]$, if and only if it is isomorphic to the coordinate Hopf algebra $O(G)$ of a simply connected affine algebraic group $G$ with $G=[G, G]$.

Here we describe the map $\kappa_{1}$, which is asserted in Theorem 4.11 to be an isomorphism, in two ways. The description of $\kappa_{0}$ is left to the reader. We use the notation (4.4).

First, at the cohomological level, $\kappa_{1}$ is identified with the composite

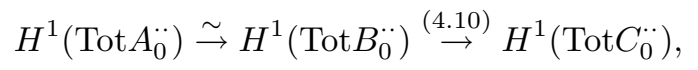

where the first isomorphism is induced from $\log : A_{0} \stackrel{\sim}{\rightarrow} B_{0}^{*}$, as is seen from the proof of Proposition 3.14. Hence we see that $\kappa_{1}$ is induced from the correspondence

$$
\left(K \#_{\sigma, \theta} H\right) \mapsto\left(\mathfrak{g}^{*} \triangleleft_{\bar{\sigma}, \bar{\theta}} \mathfrak{f}\right),
$$

where $\bar{\sigma}: \mathfrak{f} \wedge \mathfrak{f} \rightarrow \mathfrak{g}^{*}$ is determined from $\sigma: H \otimes H \rightarrow K$ by

$$
\langle\bar{\sigma}(a, b), x\rangle=\langle\sigma(1-\tau)(a \otimes b), x\rangle \quad(a, b \in \mathfrak{f}, x \in \mathfrak{g}),
$$


and $\bar{\theta}: \mathfrak{f} \rightarrow(\mathfrak{g} \wedge \mathfrak{g})^{*}$ is determined from $\theta: H \rightarrow K \otimes K$ by

$$
\langle\bar{\theta}(a), x \wedge y\rangle=\langle(1-\tau) \theta(a), x \otimes y\rangle \quad(a \in \mathfrak{f}, x, y \in \mathfrak{g}) .
$$

( $\tau$ is the twisting in the two-fold tensor product.)

The second description is suggested by M. Takeuchi, and is inspired by Mo. Theorem 7.1.10], [Mo1, Theorem 2.8]. Let

$$
(A)=K \stackrel{\iota}{\rightarrow} A \stackrel{\pi}{\rightarrow} H
$$

be a cleft extension giving rise to the fixed $\rightarrow, \rho$. As usual, we regard $A$ as a Lie algebra with the bracket defined by $[u, v]=u v-v u$ for $u, v \in A$. Write $\lambda=(1 \otimes \pi) \Delta: A \rightarrow A \otimes H$, and define $L_{A}$ to be the subvector space of $A$ consisting of the elements $u$ such that $\lambda u=u \otimes 1+1 \otimes b$ for some $b \in \mathfrak{f}$. If another $v$ in $L_{A}$ satisfies $\lambda v=v \otimes 1+1 \otimes c$ for $c \in \mathfrak{f}$, it follows that $\lambda[u, v]=[u, v] \otimes 1+1 \otimes[b, c]$. This implies that $L_{A}$ is a Lie subalgebra of $A$, and that the map $\pi-\varepsilon: L_{A} \rightarrow \mathfrak{f}$, $u \mapsto b$ is a Lie algebra map. Define $L_{A}^{+}=L_{A} \cap \operatorname{Ker} \varepsilon$, a Lie subalgebra of $L_{A}$. Then we have a sequence of Lie algebras

$$
\left(L_{A}^{+}\right)=0 \rightarrow K^{+} \stackrel{\iota}{\rightarrow} L_{A}^{+} \stackrel{\pi}{\rightarrow} \mathfrak{f} \rightarrow 0
$$

where $K^{+}$is an abelian Lie algebra. If one identifies $A=K \#_{\sigma, \theta} H$ for some $\sigma, \theta$, then it follows that

$$
L_{A}=K \otimes k+k \otimes \mathfrak{f}, \quad L_{A}^{+}=K^{+} \otimes k+k \otimes \mathfrak{f}
$$

which implies that $\left(L_{A}^{+}\right)$is exact, that is, a Lie algebra extension of $\mathfrak{f}$ by $K^{+}$. The arising action $\mathfrak{f} \otimes K^{+} \rightarrow K^{+}$is the restriction of $\rightarrow: H \otimes K \rightarrow K$, and it stabilizes the kernel $I$ of the restriction map $K^{+} \rightarrow \mathfrak{g}^{*}$ (denoted by $\left.\omega\right|_{K^{+}}$above), since $(H, K, \rightarrow, \rho)$ is induced from $\left(\mathfrak{f}, \mathfrak{g}^{*}, \rightarrow, \rho\right)$. (The induced action on $\mathfrak{g}^{*}=K^{+} / I$ coincides with the original $\rightarrow: \mathfrak{f} \otimes \mathfrak{g}^{*} \rightarrow \mathfrak{g}^{*}$. See Remark 4.5.) It follows that $\iota(I)$ is a Lie ideal of $L_{A}^{+}$. Define $\mathfrak{l}_{A}=L_{A}^{+} / \iota(I)$, the quotient Lie algebra. Then we have a Lie algebra extension

$$
\left(\mathfrak{l}_{A}\right)=\mathfrak{g}^{*} \rightarrow \mathfrak{l}_{A} \rightarrow \mathfrak{f} .
$$

On the other hand, the coalgebra $A$ is a Lie coalgebra with the cobracket defined by $\delta=(1-\tau) \Delta: A \rightarrow A \otimes A$. Since it follows from (4.18) that $L_{A}$ is a Lie subcoalgebra of $A$, we see that $L_{A}^{+}$, as well as $K^{+}$, is a Lie subcoalgebra of $A$, and hence that $\left(L_{A}^{+}\right)$is a Lie coalgebra extension, too. Furthermore, since the restriction map $K^{+} \rightarrow \mathfrak{g}^{*}$ is a Lie coalgebra map, $I$ is a Lie coideal [Mi, p. 8] of $K^{+}$, and so $\iota(I)$ is a Lie coideal of $L_{A}^{+}$. Hence, $\left(\mathfrak{l}_{A}\right)$ is a Lie coalgebra extension, too. One sees that, if $A=K \#_{\sigma, \theta} H$, then $\mathfrak{l}_{A}=\mathfrak{g}^{*} \aleph_{\bar{\sigma}, \bar{\theta}} \mathfrak{f}$ with $\bar{\sigma}, \bar{\theta}$ determined by $(4.16),(4.17)$. This implies that $\mathfrak{l}_{A}$ is a Lie bialgebra, that $\left(\mathfrak{l}_{A}\right)$ is a Lie bialgebra extension, and that $\kappa_{1}$ is induced from the correspondence

$$
(A) \mapsto\left(\mathfrak{l}_{A}\right) .
$$

Example 4.19. Let $\mathfrak{f}=k a, \mathfrak{g}=k x$ be as in Example 1.2. If a Singer pair $\left(U \mathfrak{f}, U \mathfrak{g}^{\circ}\right)=\left(k[a], k[x]^{\circ}\right)$ of Hopf algebras is induced from some Singer pair $\left(\mathfrak{f}, \mathfrak{g}^{*}\right)$ of Lie bialgebras, then

$$
\operatorname{Aut}\left(k[x]^{\circ} \# k[a]\right) \cong k, \quad \operatorname{Opext}\left(k[a], k[x]^{\circ}\right)=0 .
$$


This follows by Theorem 4.11 and Proposition 2.9, since one sees that $H^{0}\left(\operatorname{Tot} C_{0}^{\cdot}\right) \cong k, H^{1}\left(\operatorname{Tot} C_{0}^{\cdot}\right)=0$. For $c \in k$, define an algebra automorphism $\phi_{c}$ of $k[x]^{\circ} \# k[a]$, which fixes each element in $k[x]^{\circ}=k[x]^{\circ} \otimes k$, by

$$
\phi_{c}(1 \# a)=c f \# 1+1 \# a \text {, }
$$

where $f$ is the primitive element in $k[x]^{\circ}$ defined in Example 4.6. Then, $\phi_{c}$ is a Hopf algebra automorphism, and further gives an auto-equivalence of the split extension $\left(k[x]^{\circ} \# k[a]\right)$. It is seen that $c \mapsto \phi_{c}$ gives the first isomorphism above.

To show a key diagram for the proof of Theorem 4.11, we define

$$
\left(B^{\cdot}, d^{*}\right)=\operatorname{Tot} B^{\cdot}, \quad\left(C^{\cdot}, \partial^{\cdot}\right)=\operatorname{Tot} C^{\cdot},
$$

the total complexes, and let

$$
\alpha^{\cdot}: B^{\cdot} \rightarrow C^{\cdot}
$$

be the map between the cochain complexes just defined, which is induced from $\alpha$, that is, $\alpha^{n}=\bigoplus_{p+q=n} \alpha^{p q}$. Further, with the notation (4.4), let

$$
e: \operatorname{Hom}\left(H^{+}, K_{+}\right) \rightarrow \operatorname{Hom}\left(H \otimes H_{+}, K^{+} \otimes K\right)
$$

be the $H \bowtie J$-linear map induced from $\mu^{+}$in (3.12) and $\Delta_{+}$in (3.13), and let

$$
\eta: \operatorname{Hom}\left(H^{+}, K_{+}\right) \rightarrow \operatorname{Hom}\left(H \otimes \mathfrak{f}, \mathfrak{g}^{*} \otimes K\right)
$$

be the map induced from $\nu^{+}$in (4.7) and

$$
0 \rightarrow K+\stackrel{\Lambda_{+}}{\longrightarrow} \mathfrak{g}^{*} \otimes K=\operatorname{Hom}(\mathfrak{g}, K), \quad \bar{f} \mapsto(x \mapsto f x) .
$$

Since $\nu^{+}$and $\Lambda_{+}$are $H \bowtie J$-linear, $\eta$ is, too.

Proposition 4.20. With the assumption of Theorem 4.11, we have the following commutative diagram in $H \bowtie J$-Mod, in which the two rows are both exact:

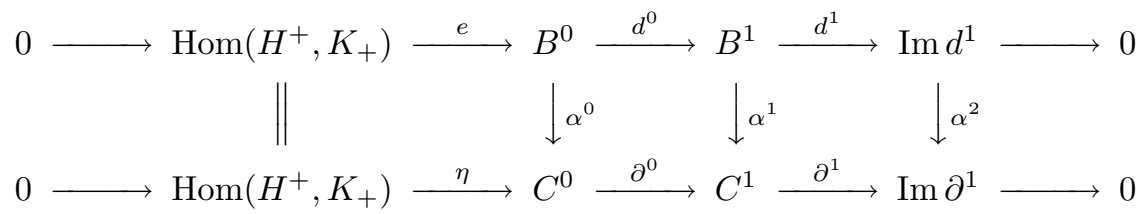

In Section 6, we will prove that, if $\operatorname{Hom}\left(H^{+}, K_{+}\right)$is deleted from each row in the diagram just above, then $\alpha^{0}, \alpha^{1}, \alpha^{2}, 0, \cdots$ give a homotopy equivalence between the deleted complexes in $H \bowtie J$-Mod. Then, by taking the $H \bowtie J$-invariants ( ) 0 , we will see that

$$
\left(\alpha^{0}\right)_{0}=\alpha_{0}^{00}: B_{0}^{00} \rightarrow C_{0}^{00}
$$

and

$$
\left(\alpha^{1}\right)_{0}=\alpha_{0}^{01} \oplus \alpha_{0}^{10}: B_{0}^{01} \oplus B_{0}^{10} \rightarrow C_{0}^{01} \oplus C_{0}^{10}
$$

induce the isomorphism (4.10), which will conclude Theorem 4.11.

Let us prove Proposition 4.20. Since the diagrams

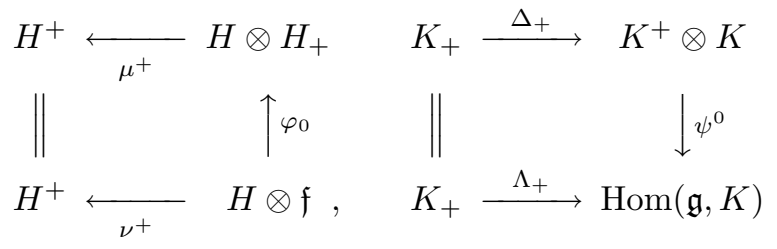


commute, the left square in (4.21) commutes. The middle and the right squares clearly commute. Recall that $\mu^{+}, \Delta_{+}$give resolutions $0 \leftarrow H^{+} \leftarrow X .(H)$ and $0 \rightarrow K_{+} \rightarrow Y^{\cdot}(H)$, respectively. Hence the first row in (4.21) is exact.

It remains to show that the second row is exact. Recall that $\nu^{+}$gives a resolution $0 \leftarrow H^{+} \leftarrow X$. (f). Hence, if we show that the sequence

$$
0 \rightarrow K_{+} \stackrel{\Lambda_{+}}{\rightarrow} \operatorname{Hom}(\mathfrak{g}, K) \rightarrow \operatorname{Hom}\left(\wedge^{2} \mathfrak{g}, K\right) \rightarrow \operatorname{Hom}\left(\wedge^{3} \mathfrak{g}, K\right)
$$

obtained by splicing $\Lambda_{+}$with the first three terms in $Y^{\cdot}\left(\mathfrak{g}^{*}\right)$ is exact, then it follows that the second row in (4.21) is exact. For one then computes the $E_{2}$-term of the spectral sequence arising from the second filtration of TotC ${ }^{*}$, and obtains

$$
E_{2}^{p q}= \begin{cases}\operatorname{Hom}\left(H^{+}, K_{+}\right) \quad(p=q=0) \\ 0 \quad(p=1 \text { or } q \neq 0) .\end{cases}
$$

(For our purpose, the terms $E_{2}^{p 0}$ with $p>1$ are not needed.) Recall that $Y^{\cdot}\left(\mathfrak{g}^{*}\right)$ is obtained by deleting the 0th term from the standard complex for computing the cohomology group $H^{\cdot}(\mathfrak{g}, K)$, and observe that the sequence (4.22) is exact at $\operatorname{Hom}(\mathfrak{g}, K)$ and at $\operatorname{Hom}\left(\wedge^{2} \mathfrak{g}, K\right)$ if and only if $H^{1}(\mathfrak{g}, K)=0$ and $H^{2}(\mathfrak{g}, K)=0$, respectively. These vanishings will be shown in Proposition 5.1 and Theorem 5.2 in the next section, which will complete the proof of Proposition 4.20.

Remark 4.23. The cochain complex $Y^{\cdot}\left(\mathfrak{g}^{*}\right)$ is not always exact at $\operatorname{Hom}\left(\wedge^{3} \mathfrak{g}, K\right)$, since it is not always true that $H^{3}(\mathfrak{g}, K)=0$. See Remark 5.9.

\section{Vanishing of Lie Algebra Cohomologies}

Let $\mathfrak{g}$ be a finite-dimensional Lie algebra in arbitrary characteristic. Suppose that $M$ is a (left or right) $\mathfrak{g}$-Lie module, or equivalently a $U \mathfrak{g}$-module. The cohomology group $H^{\cdot}(\mathfrak{g}, M)$ of $\mathfrak{g}$ with coefficients $M$ is defined to be $\operatorname{Ext}_{U \mathfrak{g}}(k, M)$ with $k$ the trivial $U \mathfrak{g}$-module. We may regard $U \mathfrak{g}^{\circ}={ }_{\mathfrak{g}}\left(U \mathfrak{g}^{\circ}\right)$ as a left $U \mathfrak{g}$-module, and $U \mathfrak{g}^{\circ}=$ $\left(U \mathfrak{g}^{\circ}\right)_{\mathfrak{g}}$ as a right $U \mathfrak{g}$-module, with respect to the transposed action of the right and left multiplication by $U \mathfrak{g}$, respectively. Hence we have two cohomology groups, $H^{\cdot}\left(\mathfrak{g}, \mathfrak{g}\left(U \mathfrak{g}^{\circ}\right)\right)$ and $H^{\cdot}\left(\mathfrak{g},\left(U \mathfrak{g}^{\circ}\right)_{\mathfrak{g}}\right)$, but these are equal to each other. For we have a category equivalence between the left $U \mathfrak{g}$-modules and the right $U \mathfrak{g}$-modules by twisting the action through the antipode of $U \mathfrak{g}$, under which $k \leftrightarrow k, \mathfrak{g}\left(U \mathfrak{g}^{\circ}\right) \leftrightarrow$ $\left(U \mathfrak{g}^{\circ}\right)_{\mathfrak{g}}$ (the antipode of $U \mathfrak{g}^{\circ}$ gives the correspondence.)

In the following proofs, we suppose $U \mathfrak{g}^{\circ}=\mathfrak{g}\left(U \mathfrak{g}^{\circ}\right)$.

Proposition 5.1. We have

$$
H^{1}\left(\mathfrak{g}, U \mathfrak{g}^{\circ}\right)=0 .
$$

Proof. Let $N \subset M$ be left $U \mathfrak{g}$-modules. We claim that, if $N$ and the quotient module $M / N$ are both locally finite, then $M$ is, too. To show this, we may assume that $M$ is finitely generated. Then $N$ as well as $M / N$ is finitely generated, since $U \mathfrak{g}$ is Noetherian. It follows that $N, M / N$ and hence $M$ are all finite-dimensional, which yields the claim.

The preceding result implies that, given a short exact sequence $0 \rightarrow U \mathfrak{g}^{\circ} \rightarrow$ $M \rightarrow k \rightarrow 0$ of left $U \mathfrak{g}$-modules, $M$ is a locally finite left $U \mathfrak{g}$-module or equivalently a right $U \mathfrak{g}^{\circ}$-comodule, and hence that the short exact sequence splits since $U \mathfrak{g}^{\circ}$ is injective as a right $U \mathfrak{g}^{\circ}$-comodule. Therefore we have

$$
H^{1}\left(\mathfrak{g}, U \mathfrak{g}^{\circ}\right)=\operatorname{Ext}_{U \mathfrak{g}}^{1}\left(k, U \mathfrak{g}^{\circ}\right)=0 .
$$


The following two theorems and their proofs are due to Hans-Jürgen Schneider.

Theorem 5.2 (Schneider). If $\operatorname{ch} k=0$, then

$$
H^{2}\left(\mathfrak{g}, U \mathfrak{g}^{\circ}\right)=0 \text {. }
$$

This follows from the next theorem. In fact, suppose that Theorem 5.3 below is known. Then, $H^{n}\left(\mathfrak{a}, U \mathfrak{g}^{\circ}\right)=0$ for $n>0$, where $\mathfrak{a}$ is the radical of $\mathfrak{g}$. Now apply the general argument in the proof of Corollary 2.11 to $(\mathfrak{h}, \mathfrak{a}, M)=\left(\mathfrak{g}, \mathfrak{a}, U \mathfrak{g}^{\circ}\right)$. Then we have in particular $H^{2}\left(\mathfrak{g}, U \mathfrak{g}^{\circ}\right) \cong H^{2}\left(\mathfrak{g} / \mathfrak{a},\left(U \mathfrak{g}^{\circ}\right)^{\mathfrak{a}}\right)$, which is zero by the second Whitehead lemma [HS, Chap. VII, Proposition 6.3] since $\left(U \mathfrak{g}^{\circ}\right)^{\mathfrak{a}}$ is a locally finite module over the semisimple Lie algebra $\mathfrak{g} / \mathfrak{a}$. The argument shows also that $H^{1}\left(\mathfrak{g}, U \mathfrak{g}^{\circ}\right)=0$ in characteristic zero. (Schneider showed this reduction originally more directly by a dimension shift argument for the Ext groups of an augmented algebra.)

Theorem 5.3 (Schneider). Suppose $\operatorname{ch} k=0$. Let $\mathfrak{a}$ be the radical of $\mathfrak{g}$. Then $U \mathfrak{g}^{\circ}$ is injective as a left $U \mathfrak{a}$-module.

Proof of Theorem 5.3. To reduce the problem to the case where $k$ is algebraically closed, we need a base extension argument. Let $\bar{k}$ be an algebraic closure of $k$. Define $\mathfrak{g}_{\bar{k}}=\mathfrak{g} \otimes \bar{k}$, the $\bar{k}$-Lie algebra, and notice that $U\left(\mathfrak{g}_{\bar{k}}\right)=U \mathfrak{g} \otimes \bar{k}$.

Claim 5.4. The two-sided Ug-linear injection

$$
U \mathfrak{g}^{\circ} \rightarrow U\left(\mathfrak{g}_{\bar{k}}\right)^{\circ}
$$

induced from the natural injection $U \mathfrak{g}^{*}=\operatorname{Hom}(U \mathfrak{g}, k) \rightarrow \operatorname{Hom}(U \mathfrak{g}, \bar{k})=U\left(\mathfrak{g}_{\bar{k}}\right)^{*}$ (the $\bar{k}$-linear dual) splits.

Proof. In fact, we shall see that this holds true for a finitely generated algebra $J$ in arbitrary characteristic. Write $J_{\bar{k}}=J \otimes \bar{k}$.

A $k$-linear projection $\bar{k} \rightarrow k$ induces a two-sided $J$-linear splitting $J_{\bar{k}}{ }^{*} \rightarrow J^{*}$ of the natural injection $J^{*} \rightarrow J_{\bar{k}^{*}}{ }^{*}$. To see that this maps $J_{\bar{k}}{ }^{\circ}$ into $J^{\circ}$, we show that, for each cofinite ideal $I$ of $J_{\bar{k}}, I \cap J$ is a cofinite ideal of $J$. Notice that $I$ is the kernel of some matrix representation $J_{\bar{k}} \rightarrow M_{r}(\bar{k})$ over $\bar{k}$. Since $J$ is finitely generated, every matrix representation of $J_{\bar{k}}$ is defined over some finite extension field $l$ of $k$. Hence, $I=I^{\prime} \otimes_{l} \bar{k}$ for some ideal $I^{\prime}$ of $J \otimes l$. Trivially, $I^{\prime}$ is cofinite over $l$, hence over $k$. Since $I \cap J=I^{\prime} \cap J, I \cap J$ is cofinite in $J$, as desired.

Now, by [B, Chap. I, Sect. $\left.5, \mathrm{~N}^{\circ} 5\right], \mathfrak{a}_{\bar{k}}:=\mathfrak{a} \otimes \bar{k}$ is the radical of $\mathfrak{g}_{\bar{k}}$. Suppose it is known that $U\left(\mathfrak{g}_{\bar{k}}\right)^{\circ}$ is injective as a left $U\left(\mathfrak{a}_{\bar{k}}\right)$-module. Since one sees easily that $U\left(\mathfrak{g}_{\bar{k}}\right)^{\circ}$ is then injective as a left $U \mathfrak{a}$-module, it will follow from Claim 5.4 that $U \mathfrak{g}^{\circ}$ is an injective left $U \mathfrak{a}$-module. Hence we may suppose in the following that $k$ is an algebraically closed field (of characteristic zero).

There is a nilpotent Lie subalgebra $\mathfrak{n}$ of $\mathfrak{a}$ such that $\mathfrak{a}=[\mathfrak{a}, \mathfrak{a}]+\mathfrak{n}$ (apply the result [H1, p. 610, lines 32-33] for $L$ to $\mathfrak{a})$. Let $s \leq m \leq n$ be the dimensions of $[\mathfrak{a}, \mathfrak{a}] \subset[\mathfrak{g}, \mathfrak{a}] \subset \mathfrak{a}$, respectively.

Claim 5.5 (cf. [H, p. 516], [H1, p. 610]). There is a basis $x_{1}, x_{2}, \ldots, x_{n}$ of a such that

1) $x_{1}, \ldots, x_{s} \operatorname{span}[\mathfrak{a}, \mathfrak{a}]$;

2) $x_{1}, \ldots, x_{m} \operatorname{span}[\mathfrak{g}, \mathfrak{a}]$;

3) $x_{s+1}, \ldots, x_{n}$ are in $\mathfrak{n}$;

4) $\left[x_{p}, x_{q}\right] \in \sum_{i<p} k x_{i}$, if $1 \leq p<q \leq m$. 
Proof. Since $[\mathfrak{a}, \mathfrak{a}] \subset[\mathfrak{g}, \mathfrak{a}]$ are ideals of the solvable Lie algebra $\mathfrak{a}$, it follows from the Lie theorem [B, Chap. I, Sect. 5, Corollary 2] that there is a series of ideals in $\mathfrak{a}$,

$$
0=\mathfrak{a}_{0} \subset \mathfrak{a}_{1} \subset \cdots \subset \mathfrak{a}_{s}=[\mathfrak{a}, \mathfrak{a}] \subset \mathfrak{a}_{s+1} \subset \cdots \subset \mathfrak{a}_{m}=[\mathfrak{g}, \mathfrak{a}]
$$

with $\operatorname{dim} \mathfrak{a}_{i}=i(0 \leq i \leq m)$. Notice that $[\mathfrak{g}, \mathfrak{a}]$ is nilpotent since it is included in the nilpotent Lie algebra $[\mathfrak{g}, \mathfrak{g}] \cap \mathfrak{a}$ (see [B, Chap. I, Sect. 5, Theorem 1]). Regard each $\mathfrak{a}_{i} / \mathfrak{a}_{i-1}$ as a $[\mathfrak{g}, \mathfrak{a}]$-Lie module via adjoint, and apply the Engel theorem $\mathrm{B}$, Chap. I, Sect. 4, Theorem 1]. Then one sees that $\mathfrak{a}_{i} / \mathfrak{a}_{i-1}$ is a trivial $[\mathfrak{g}, \mathfrak{a}]$-module. Let $x_{i}$ be a basis of $\mathfrak{a}_{i}$ modulo $\mathfrak{a}_{i-1}$. Then, $\left[x, x_{i}\right] \in \mathfrak{a}_{i-1}$ for $x \in[\mathfrak{g}, \mathfrak{a}]$. Thus we obtain a basis $x_{1}, \ldots, x_{m}$ of $[\mathfrak{g}, \mathfrak{a}]$ satisfying the conditions 1$), 2$ ) and 4 ).

Since $\mathfrak{a}=[\mathfrak{a}, \mathfrak{a}]+\mathfrak{n}$ and $[\mathfrak{a}, \mathfrak{a}]$ is spanned by $x_{1}, \ldots, x_{s}$, one can choose an element $x_{q}^{\prime} \in \mathfrak{n}$, for each $q$ with $s<q \leq m$, in the form

$$
x_{q}^{\prime}=\sum_{i=1}^{s} \alpha_{i q} x_{i}+x_{q}
$$

where $\alpha_{i q} \in k$. Clearly, $x_{1}, \ldots, x_{s}, x_{s+1}^{\prime}, \ldots, x_{m}^{\prime}$ form a basis of $[\mathfrak{g}, \mathfrak{a}]$. It is not difficult to see that these still satisfy the condition 4 ). Now, complete the basis of $[\mathfrak{g}, \mathfrak{a}]$ just obtained to a basis of $\mathfrak{a}$, by adding elements in $\mathfrak{n}$. Then the claim is established.

The above proof shows that the basis can be chosen further so that $\left[x, \sum_{i \leq q} k x_{i}\right] \subset \sum_{i \leq q} k x_{i}$ for all $x \in \mathfrak{a}$ and $1 \leq q \leq m$ (cf. [L, p. 52]). But this property will not be needed in our argument.

Write $\mathfrak{h}=\mathfrak{g} / \mathfrak{a}$, the quotient semisimple Lie algebra. By the Levi-Malcev theorem B, Chap. I, Sect. 8, Theorem 5], the quotient map $\mathfrak{g} \rightarrow \mathfrak{h}$ splits. Hence the natural surjection $\pi: U \mathfrak{g} \rightarrow U \mathfrak{h}$ has a Hopf algebra splitting $\gamma: U \mathfrak{h} \rightarrow U \mathfrak{g}$. Passing to the Hopf duals, we have Hopf algebra maps $\gamma^{\circ}: U \mathfrak{g}^{\circ} \rightarrow U \mathfrak{h}^{\circ}$ and $\pi^{\circ}: U \mathfrak{h}^{\circ} \rightarrow U \mathfrak{g}^{\circ}$ with $\gamma^{\circ} \pi^{\circ}=1$. Define

$$
R=\left\{f \in U \mathfrak{g}^{\circ}:\left(\gamma^{\circ} \otimes 1\right) \Delta(f)=1 \otimes f\right\},
$$

the left $U \mathfrak{h}^{\circ}$-coinvariants along $\gamma^{\circ}$. Then, $R$ is a right coideal subalgebra of $U \mathfrak{g}^{\circ}$, or in other words a subalgebra which is a left $U \mathfrak{g}$-submodule of $U \mathfrak{g}^{\circ}$, and so it is in particular a left $U \mathfrak{a}$-submodule of $U \mathfrak{g}^{\circ}$. By [BCM, Theorem 4.14], there is an isomorphism

$$
U \mathfrak{h}^{\circ} \otimes R \cong U \mathfrak{g}^{\circ},
$$

given by $f \otimes g \mapsto \pi^{\circ}(f) g$. It is easy to see that this is left $U \mathfrak{a}$-linear, if we let $U \mathfrak{a}$ act on the factor $R$ in $U \mathfrak{h}^{\circ} \otimes R$. Hence we have only to show that the left $U \mathfrak{a}$-module $R$ is injective. For the isomorphism (5.6) then yields that $U \mathfrak{g}^{\circ}$ is $U \mathfrak{a}$-injective, since $U \mathfrak{a}$ is Noetherian.

Let $\rho: U \mathfrak{g}^{\circ} \rightarrow U \mathfrak{a}^{\circ}$ be the Hopf algebra map dual to the inclusion $U \mathfrak{a} \hookrightarrow U \mathfrak{g}$. The image $\operatorname{Im} \rho$ of $\rho$ is trivially a Hopf subalgebra of $U \mathfrak{a}^{\circ}$, and hence it is in particular a left $U \mathfrak{a}$-submodule of $U \mathfrak{a}^{\circ}$.

Claim 5.7. $\left.\rho\right|_{R}: R \rightarrow \operatorname{Im} \rho$ is a left Ua-linear isomorphism.

Proof. Clearly, $\left.\rho\right|_{R}$ is left $U$ a-linear. It follows from the isomorphism (5.6) that this is a surjection. To show that this is an injection, let $f \in R$ with $\rho(f)=0$. There 
is an isomorphism $U \mathfrak{h} \otimes U \mathfrak{a} \cong U \mathfrak{g}$ given by $x \otimes a \mapsto \gamma(x) a$. We have

$$
\begin{aligned}
\langle f, \gamma(x) a\rangle & =\varepsilon(x)\langle f, a\rangle & & (\text { since } f \in R) \\
& =0 & & (\text { since } \rho(f)=0) .
\end{aligned}
$$

We conclude that $\left.\rho\right|_{R}$ is an isomorphism.

Donkin [Don, Proposition 2.2.2] shows that an essential extension of a locally finite left $U \mathfrak{a}$-module is locally finite. This implies that the left $U \mathfrak{a}$-module $U \mathfrak{a}^{\circ}$ is injective (consider the injective hull of $U \mathfrak{a}^{\circ}$ in $U \mathfrak{a}^{*}$ ). Hence, for our purpose, it suffices to show that $\operatorname{Im} \rho(\cong R)$ is injective as a right $U \mathfrak{a}^{\circ}$-comodule. For this implies that $R$ is a direct summand of the injective left $U \mathfrak{a}$-module $U \mathfrak{a}^{\circ}$, so that $R$ is, too.

Claim 5.8. $\operatorname{Im} \rho$ includes a right coideal subalgebra $E$ of $U \mathfrak{a}^{\circ}$ that is injective as a right $U \mathfrak{a}^{\circ}$-comodule.

Proof. Choose a basis $x_{1}, \ldots, x_{n}$ of $\mathfrak{a}$ as in Claim 5.5. Define elements $f_{1}, \ldots, f_{n}$ in $U \mathfrak{a}^{*}$ by

$$
\left\langle f_{i}, x_{n}^{e_{n}} \ldots x_{1}^{e_{1}}\right\rangle= \begin{cases}1, & \text { if } e_{i}=1 \text { and } e_{j}=0 \text { for } j \neq i, \\ 0, & \text { otherwise. }\end{cases}
$$

Then it follows by [H] Proposition 2] that each $f_{i} \in \operatorname{Im} \rho$, since the basis satisfies the conditions 2) and 4). Define $E=k\left[f_{1}, \ldots, f_{n}\right]$, the subalgebra of $U \mathfrak{a}^{\circ}$ generated by $f_{1}, \ldots, f_{n}$. Then it follows by applying the results [H1, pp. 610-611] for $L$ to $\mathfrak{a}$ (see also [L Proposition 1.3]) that $E$ is a right coideal subalgebra of $U \mathfrak{a}^{\circ}$, and is an injective left $U \mathfrak{a}$-module, since the basis satisfies the conditions 1), 3) and 4). In fact, $\exp f$, where $f$ is a $k$-linear combination of $f_{i}(s<i \leq n)$, are linearly independent in $U \mathfrak{a}^{\circ}$ and form a group, say $\Gamma$, so that $U \mathfrak{a}^{\circ}=E \otimes k \Gamma$. Since each $x_{j}$ $(1 \leq j \leq n)$ acts on $U \mathfrak{a}^{\circ}$ by a left $E$-linear combination of $\frac{\partial}{\partial f_{i}}(1 \leq i \leq n)$, we see that $E$ is a left $U \mathfrak{a}$-submodule of $U \mathfrak{a}^{\circ}$ and is further its direct summand, where a projection is induced from $\exp f \mapsto \delta_{0 f}, k \Gamma \rightarrow k$.

We know now that $E \subset \operatorname{Im} \rho$ are right $U \mathfrak{a}^{\circ}$-comodule algebras with $E$ injective as a $U \mathfrak{a}^{\circ}$-comodule. It follows by [Doi, Theorem 1.6, (3) $\Rightarrow(1)$ ] that there is a $U \mathfrak{a}^{\circ}$ colinear map $\phi: U \mathfrak{a}^{\circ} \rightarrow E$ such that $\phi(1)=1$. Composing $\phi$ with the inclusion $E \hookrightarrow \operatorname{Im} \rho$, we have a $U \mathfrak{a}^{\circ}$-colinear map $\phi^{\prime}: U \mathfrak{a}^{\circ} \rightarrow \operatorname{Im} \rho$ such that $\phi^{\prime}(1)=1$. It follows again by [Doi, Theorem $1.6(1) \Rightarrow(3)]$ that $\operatorname{Im} \rho$ is an injective right $U \mathfrak{a}^{\circ}$ comodule. This completes the proof of Theorem 5.3.

Remark 5.9. Suppose $\operatorname{ch} k=0$, and let $\mathfrak{g}$ be a finite-dimensional Lie algebra. Koszul [K, Théorème 7] showed that, if $\mathfrak{g}$ is solvable, then $H^{n}\left(\mathfrak{g}, U \mathfrak{g}^{\circ}\right)=0$ for all $n>0$. Theorem 5.2 generalized this result in dimension $n=2$. But the result cannot be generalized in dimension $n=3$. In fact, suppose that $\mathfrak{g}$ is semisimple. Then the trivial $U \mathfrak{g}$-module $k$ is a direct summand of $U \mathfrak{g}^{\circ}$, so that each $H^{n}(\mathfrak{g}, k)$ is a vector subspace of $H^{n}\left(\mathfrak{g}, U \mathfrak{g}^{\circ}\right)$. But, $H^{3}(\mathfrak{g}, k) \neq 0$ by [HS, Chap. VII, Exercise $6.2]$, and so $H^{3}\left(\mathfrak{g}, U \mathfrak{g}^{\circ}\right) \neq 0$.

\section{Continuous Modules}

To complete the proof of Theorem 4.11, we will show that $\alpha^{0}, \alpha^{1}, \alpha^{2}, 0, \cdots$ in (4.21) give a homotopy equivalence between the deleted complexes in $H \bowtie J$-Mod, the category of left $H \bowtie J$-modules. For this purpose, we will define a category $\mathcal{D}$ 
which consists of left $H \bowtie J$-modules with some topology. It will be seen that the diagram (4.21) is in $\mathcal{D}$, and that $B^{i}, C^{i}(i=0,1)$ therein look like injective objects in $\mathcal{D}$.

We shall use the term 'topological vector space' in a stricter sense than it is used normally:

Definition 6.1 T1, p. 507]. A topological vector space is a vector space $V$ with a topology, called a linear topology, such that

1) for each $w \in V$, the translation $v \mapsto v+w, V \rightarrow V$ is continuous, and

2) $V$ has a basis of neighborhoods of 0 consisting of vector subspaces, which we call a topological basis.

Every vector space is a topological vector space with the discrete topology. We always regard $k$ as a discrete topological vector space.

Let $V, V^{\prime}$ be topological vector spaces. We denote by $\operatorname{Hom}_{\mathrm{c}}\left(V, V^{\prime}\right)$ the vector subspace of $\operatorname{Hom}\left(V, V^{\prime}\right)$, which consists of the continuous linear maps $V \rightarrow V^{\prime}$.

For a vector space $Z$ (either topological or not), we identify

$$
\operatorname{Hom}(Z, V)=\prod_{\operatorname{dim} Z} V
$$

the direct product of $\operatorname{dim} Z$ copies of $V$, and regard $\operatorname{Hom}(Z, V)$ as a topological vector space with the direct product topology.

We do not adopt the tensor product topology [T1, 1.5]. In addition, we consider only the tensor product of $V$ with a discrete vector space $Z$. Identify

$$
V \otimes Z \quad \text { or } Z \otimes V=\bigoplus_{\operatorname{dim} Z} V
$$

the direct sum of $\operatorname{dim} Z$ copies of $V$, regard this as a topological vector space with the direct sum topology [T1, 1.2], and denote this by $V \otimes(Z)$ or $(Z) \otimes V$. Thus, if $\left\{z_{\lambda}\right\}$ is a (free) basis of $Z$ and $\left\{V_{\mu}\right\}$ is a topological basis of $V$, then $V \otimes(Z)$ has a topological basis consisting of all $\bigoplus_{\lambda} V_{\lambda} \otimes k z_{\lambda}$, where $V_{\lambda}$ is an arbitrary element in $\left\{V_{\mu}\right\}$. (This topology is finer than the tensor product topology.)

Proposition 6.2. Let $V$ be a topological vector space, and let $Z, W$ be discrete vector spaces. Then there is a natural linear isomorphism

$$
\operatorname{Hom}_{\mathrm{c}}(V \otimes(Z), W) \cong \operatorname{Hom}_{\mathrm{c}}(V, \operatorname{Hom}(Z, W)) .
$$

Proof. Let $\left\{z_{\lambda}\right\},\left\{V_{\mu}\right\}$ be as above. Notice that $\operatorname{Hom}(Z, W)$ has a topological basis consisting of $\operatorname{Hom}\left(Z / Z^{\prime}, W\right)$, where $Z^{\prime} \subset Z$ are finite-dimensional vector subspaces.

There is a canonical isomorphism

$$
\phi \leftrightarrow \hat{\phi}, \operatorname{Hom}(V \otimes Z, W) \cong \operatorname{Hom}(V, \operatorname{Hom}(Z, W))
$$

such that $\phi(v \otimes z)=\hat{\phi}(v)(z)$ for $v \in V, z \in Z$.

Suppose $\phi$ is continuous. Then for each $\lambda$, there exists $V_{\lambda}$ in $\left\{V_{\mu}\right\}$ such that $\phi\left(V_{\lambda} \otimes k z_{\lambda}\right)=0$. Let $Z^{\prime} \subset Z$ be a finite-dimensional vector subspace. Then there are finitely many $z_{\lambda_{1}}, \ldots, z_{\lambda_{r}}$ in $\left\{z_{\lambda}\right\}$ such that $Z^{\prime} \subset \sum_{i=1}^{r} k z_{\lambda_{i}}$. Choose $V_{\mu}$ from $\left\{V_{\mu}\right\}$ such that $V_{\mu} \subset \bigcap_{i=1}^{r} V_{\lambda_{i}}$. Then, $\hat{\phi}\left(V_{\mu}\right) \subset \operatorname{Hom}\left(Z / Z^{\prime}, W\right)$, and so that $\hat{\phi}$ is continuous.

Conversely, suppose $\hat{\phi}$ is continuous. Then for each $\lambda$, there exists $V_{\lambda}$ in $\left\{V_{\mu}\right\}$ such that $\phi\left(V_{\lambda} \otimes k z_{\lambda}\right)=\hat{\phi}\left(V_{\lambda}\right)\left(z_{\lambda}\right)=0$, which implies that $\phi$ is continuous. 
Corollary 6.3. Let $V, Z, W$ be as above. Suppose that $V^{\prime}$ is a topological vector subspace of $V$ (in the sense that $V^{\prime}$ is a topological subspace as well as a vector subspace). Then the restriction map

$$
\operatorname{Hom}_{\mathrm{c}}(V, \operatorname{Hom}(Z, W)) \rightarrow \operatorname{Hom}_{\mathrm{c}}\left(V^{\prime}, \operatorname{Hom}(Z, W)\right)
$$

is a surjection.

Proof. Notice that $\mathcal{V}^{\prime}:=V^{\prime} \otimes(Z)$ is a topological vector subspace of $\mathcal{V}:=V \otimes(Z)$. Thus, if $\left\{\mathcal{V}_{\mu}\right\}$ is a topological basis of $\mathcal{V}$, then $\mathcal{V}_{\mu}^{\prime}:=\mathcal{V}_{\mu} \cap \mathcal{V}^{\prime}$ form a topological basis of $\mathcal{V}^{\prime}$. By the preceding proposition, the restriction map above is identified with the restriction map

$$
\operatorname{Hom}_{\mathrm{c}}(\mathcal{V}, W) \rightarrow \operatorname{Hom}_{\mathrm{c}}\left(\mathcal{V}^{\prime}, W\right) .
$$

This is indeed a surjection, since it equals the inductive limit of the surjections

$$
\operatorname{Hom}\left(\mathcal{V} / \mathcal{V}_{\mu}, W\right) \rightarrow \operatorname{Hom}\left(\mathcal{V}^{\prime} / \mathcal{V}_{\mu}^{\prime}, W\right)
$$

induced from the natural injections $\mathcal{V}^{\prime} / \mathcal{V}_{\mu}^{\prime} \rightarrow \mathcal{V} / \mathcal{V}_{\mu}$.

In the sequel, modules are meant to be left modules unless otherwise stated.

Let $J$ be a Hopf algebra. Then, $J$ is a topological algebra in the sense of [T1, p. 521], which has a topological basis $\left\{I_{\gamma}\right\}$ consisting of all cofinite ideals $I_{\gamma}$ in $J$.

Definition 6.4. A topological vector space $M$ is called a continuous $J$-module, if $M$ is further a $J$-module such that

1) the $J$-action $J \otimes(M) \rightarrow M$ is continuous, or equivalently its representation $J \rightarrow \operatorname{Hom}(M, M)$, is continuous, and

2) $M$ has a topological basis consisting of $J$-submodules.

Explicitly, the condition 1 holds if and only if, for any $m \in M$ and an open $J$-submodule $M^{\prime} \subset M$, there exists some $I_{\gamma}$ such that $I_{\gamma} m \subset M^{\prime}$. Hence a discrete $J$-module is continuous if and only if it is locally finite. We regard a locally finite $J$-module as a discrete continuous $J$-module.

Proposition 6.5. Let $M$ be a continuous $J$-module, and let $P, Q$ be locally finite $J$-modules.

1) $\operatorname{Hom}(P, Q)$ is a continuous $J$-module under the conjugate action.

2) $M \otimes(P)$ is a continuous $J$-module under the diagonal action.

3) There is a natural linear isomorphism

$$
\operatorname{Hom}_{\mathrm{c}, J}(M \otimes(P), Q) \cong \operatorname{Hom}_{\mathrm{c}, J}(M, \operatorname{Hom}(P, Q)),
$$

where $\mathrm{Hom}_{\mathrm{c}, J}$ indicates the vector space of the continuous $J$-linear maps.

Proof. 1) Notice that $\operatorname{Hom}(P, Q)$ has a topological basis consisting of the $J$-submodules $\operatorname{Hom}\left(P / P^{\prime}, Q\right)$, where $P^{\prime}$ are finite-dimensional $J$-submodules of $P$. Let $P^{\prime}$ be such and $\phi \in \operatorname{Hom}(P, Q)$. Then, $\phi\left(P^{\prime}\right)$ is included in a finite-dimensional $J$ submodule $Q^{\prime}$ of $Q$. Since $\operatorname{Hom}\left(P^{\prime}, Q^{\prime}\right)$ is a finite-dimensional $J$-module containing $\left.\phi\right|_{P^{\prime}}$, there is a cofinite ideal $I_{\gamma}$ of $J$ such that $I_{\gamma}\left(\left.\phi\right|_{P^{\prime}}\right)=0$ or $I_{\gamma} \phi \subset \operatorname{Hom}\left(P / P^{\prime}, Q\right)$. This shows that the $J$-module $\operatorname{Hom}(P . Q)$ is continuous.

2) Write $P=\bigcup_{\lambda} P_{\lambda}$, a directed union of finite-dimensional $J$-submodules $P_{\lambda}$ of $P$. One sees that the $J$-submodules in the form $\sum_{\lambda} M_{\lambda} \otimes P_{\lambda}$, where $M_{\lambda}$ is an open $J$-submodule of $M$, constitute a topological basis of $M \otimes(P)$. Let $\sum_{i=1}^{r} m_{i} \otimes p_{i}$ be an element in $M \otimes(P)$. There is $\lambda$ such that $p_{i} \in P_{\lambda}$ for $1 \leq i \leq r$. Let $M_{\lambda}$ be as above. Then there are cofinite ideals $I_{1}, I_{2}$ in $J$ such that $I_{1} m_{i} \subset M_{\lambda}, I_{2} p_{i}=0$ for 
all $1 \leq i \leq r$. If we define $I=\Delta^{-1}\left(I_{1} \otimes J+J \otimes I_{2}\right)$, then $I$ is a cofinite ideal of $J$ and $I\left(\sum_{i=1}^{r} m_{i} \otimes p_{i}\right) \subset \sum_{i=1}^{r} I_{1} m_{i} \otimes J p_{i} \subset M_{\lambda} \otimes P_{\lambda}$, which means that $M \otimes(P)$ is continuous.

3) We know by Proposition 6.2 that there is a natural isomorphism $\phi \leftrightarrow \hat{\phi}$, $\operatorname{Hom}_{\mathrm{c}}(M \otimes(P), Q) \cong \operatorname{Hom}_{\mathrm{c}}(M, \operatorname{Hom}(P, Q))$. It is standard to see that $\phi$ is $J$-linear if and only if $\hat{\phi}$ is.

Define $K=J^{\circ}$, the dual Hopf algebra of $J$.

Proposition 6.6. Let $M$ be a continuous $J$-module. Let $Z$ be a discrete vector space, and regard $Z \otimes K$, via the right comultiplication by $K$ on the factor $K$, as a right $K$-comodule or a locally finite $J$-module. Then there is a natural linear isomorphism

$$
\operatorname{Hom}_{\mathrm{c}, J}(M, Z \otimes K) \cong \operatorname{Hom}_{\mathrm{c}}(M, Z)
$$

given by $\phi \mapsto(1 \otimes \varepsilon) \phi$.

Proof. Let $\left\{M_{\mu}\right\}$ be a topological basis of $M$ consisting of $J$-submodules. Each quotient $M / M_{\mu}$ is a locally finite $J$-module. Moreover, we easily see that

$$
\operatorname{Hom}_{\mathrm{c}, J}(M, Z \otimes K)=\bigcup_{\mu} \operatorname{Hom}_{J}\left(M / M_{\mu}, Z \otimes K\right) .
$$

Since $\operatorname{Hom}_{J}\left(M / M_{\mu}, Z \otimes K\right)$ equals the vector space of all $K$-colinear maps $M / M_{\mu} \rightarrow$ $Z \otimes K$, it is isomorphic to $\operatorname{Hom}\left(M / M_{\mu}, Z\right)$ via $\phi \mapsto(a \otimes \varepsilon) \phi$. Hence the right-hand side of (6.7) is isomorphic to

$$
\bigcup_{\mu} \operatorname{Hom}\left(M / M_{\mu}, Z\right)=\operatorname{Hom}_{\mathrm{c}}(M, Z),
$$

which completes the proof.

Let $(H, J, \triangleright, \triangleleft)$ be a matched pair of cocommutative Hopf algebras (Definition 2.1). Recall that the Hopf algebra $H \bowtie J$ is constructed. Continue to regard $J$ as a topological algebra.

Definition 6.8. We define a category $\mathcal{D}$ as follows. An object of $\mathcal{D}$ is an $H \bowtie J$ module $M$ such that

1) $M$ has a topology so that $M$ is a continuous $J$-module (provided the $H \bowtie J$ action is restricted to $J=k \otimes J)$, and

2 ) the $H$-action $(H) \otimes M \rightarrow M$, obtained by restriction, is continuous, or equivalently, for each $a \in H$, its action $a \cdot M \rightarrow M$ is continuous.

A morphism in $\mathcal{D}$ is a continuous $H \bowtie J$-linear map. For objects $M, M^{\prime}$ in $\mathcal{D}$, let $\operatorname{Hom}_{\mathcal{D}}\left(M, M^{\prime}\right)$ denote the vector space of all morphisms $M \rightarrow M^{\prime}$ in $\mathcal{D}$.

One sees that the category $\mathcal{D}$ is additive, but not abelian.

Proposition 6.9. Let $M$ be an object of $\mathcal{D}$, and let $N$ be an $H \bowtie J$-submodule of $M$. Then, $N$ is an object of $\mathcal{D}$, equipped with the subtopology. The quotient $H \bowtie J$-module $M / N$ is an object of $\mathcal{D}$, equipped with the quotient topology.

Proof. Notice that $J \otimes(N),(H) \otimes N$ are topological subspaces of $J \otimes(M),(H) \otimes M$, respectively, and that $J \otimes(M / N),(H) \otimes M / N$ are quotient topological spaces of $J \otimes(M),(H) \otimes M$, respectively. Then the proposition follows easily. 
Lemma 6.10. Let $M, N$ be objects in $\mathcal{D}$.

1) If $M, N$ are both discrete, then the topological vector space $\operatorname{Hom}(M, N)$ with the conjugate $H \bowtie J$-action is an object in $\mathcal{D}$.

2) If $N$ is discrete, then the topological vector space $M \otimes(N)$ with the diagonal $H \bowtie J$-action is an object in $\mathcal{D}$.

Proof. It suffices by Proposition 6.5 that each action by $a(\in H)$ is continuous.

1) Write $\Delta a=\sum_{i=1}^{r} a_{i} \otimes a_{i}^{\prime}$. Let $V \subset M$ be a finite-dimensional vector subspace, and define $W=\sum_{i=1}^{\bar{r}} S\left(a_{i}^{\prime}\right) V$, which is also finite-dimensional. Then for any $\phi \in \operatorname{Hom}(M, N)$ with $\phi(W)=0$, we have

$$
(a \phi)(V)=\sum_{i=1}^{r} a_{i} \phi\left(S\left(a_{i}^{\prime}\right) V\right) \subset \sum_{i=1}^{r} a_{i} \phi(W)=0,
$$

which shows that the action by $a$ is continuous.

2) It suffices further to show that $m \otimes n \mapsto a m \otimes a^{\prime} n, M \otimes(N) \rightarrow M \otimes(N)$ is continuous, where $a, a^{\prime} \in H$. Let $\left\{n_{\lambda}\right\}$ be a basis of $N$. Choose an open vector subspace $V_{\lambda} \subset M$ for each $\lambda$, and form $V=\bigoplus_{\lambda} V_{\lambda} \otimes k n_{\lambda}$. For each $\lambda$, there are finitely many $n_{\lambda_{1}}, \ldots, n_{\lambda_{r}}$ in $\left\{n_{\lambda}\right\}$ such that $a^{\prime} n_{\lambda} \in \sum_{i=1}^{r} k n_{\lambda_{i}}$. Since the action by $a$ is continuous, there is an open vector subspace $W_{\lambda} \subset M$ such that $a W_{\lambda} \subset \bigcap_{i=1}^{r} V_{\lambda_{i}}$. We see that $\left(a \otimes a^{\prime}\right)\left(\bigoplus_{\lambda} W_{\lambda} \otimes k n_{\lambda}\right) \subset V$, which shows that $m \otimes n \mapsto a m \otimes a^{\prime} n$ is continuous.

We assume in addition that $H$ is a locally finite $J$-module under $\triangleright$, and write $K=J^{\circ}$. It follows by Lemma 4.1 that a Singer pair $(H, K, \rightarrow, \rho)$ of Hopf algebras is induced, and accordingly the category $\mathcal{C}=\mathcal{C}(H, K, \rightarrow, \rho)$ is defined as in Definition 3.4 .

An object $M$ in $\mathcal{C}$ is regarded as an $H \bowtie J$-module by Lemma 4.1. Moreover, one sees that $M$ is an object in $\mathcal{D}$, equipped with the discrete topology. Conversely, any discrete object in $\mathcal{D}$ may be regarded as an object in $\mathcal{C}$.

Corollary 6.11. 1) $\mathcal{C}$ is the full subcategory of $\mathcal{D}$ consisting of the discrete objects.

2) $\operatorname{Hom}($, ) gives a biadditive functor

$$
\operatorname{Hom}(,): \mathcal{C}^{\mathrm{op}} \times \mathcal{C} \rightarrow \mathcal{D} .
$$

Proof. 1) This follows from the preceding argument.

2) This follows from Part 1 and Lemma 6.10 1).

Corollary 6.12. Let $P$ be a right $K$-comodule, and let $Q$ be a left $H$-module. Define the objects $F(P)=H \otimes P$ and $G(Q)=Q \otimes K$ in $\mathcal{C}$ as in Lemmas 3.5 and 3.7, respectively. Then, $\operatorname{Hom}(F(P), G(Q))$ is an object in $\mathcal{D}$.

Proposition 6.13. Let $P, Q$ be as above. Then for each $M \in \mathcal{D}$, there is a linear isomorphism

$$
\operatorname{Hom}_{\mathrm{c}}(M, \operatorname{Hom}(P, Q)) \cong \operatorname{Hom}_{\mathcal{D}}(M, \operatorname{Hom}(F(P), G(Q))),
$$

which is natural in $M$.

Proof. Apply Proposition 6.53$)$ to $M, \mathcal{P}:=F(P), \mathcal{Q}:=G(Q)$. Then one sees that the isomorphism given therein induces

$$
\operatorname{Hom}_{\mathcal{D}}(M \otimes(\mathcal{P}), \mathcal{Q}) \cong \operatorname{Hom}_{\mathcal{D}}(M, \operatorname{Hom}(\mathcal{P}, \mathcal{Q})) .
$$


We claim that there is a natural linear isomorphism

$$
\operatorname{Hom}_{\mathcal{D}}(M \otimes(\mathcal{P}), \mathcal{Q}) \cong \operatorname{Hom}_{\mathrm{c}, J}(M \otimes(P), Q \otimes K) .
$$

This, together with Propositions 6.6 and 6.2, will yield the proposition. We equip the topological vector space

$$
L:=M \otimes(\mathcal{P})=M \otimes(H \otimes P)
$$

with an alternative $H \bowtie J$-module structure, by defining

$$
\begin{aligned}
& b(m \otimes a \otimes p)=m \otimes b a \otimes p, \\
& x(m \otimes a \otimes p)=\sum\left(x_{(1)} \triangleleft a_{(1)}\right) m \otimes\left(x_{(2)} \triangleright a_{(2)}\right) \otimes\left(x_{(3)} \triangleleft a_{(3)}\right) p
\end{aligned}
$$

for $b \in H, x \in J, m \otimes a \otimes p \in L$. Then we see that the map

$$
\zeta: L \rightarrow M \otimes(H \otimes P), \quad \zeta(m \otimes a \otimes p)=\sum a_{(1)} m \otimes a_{(2)} \otimes p
$$

is $H \bowtie J$-linear, using the assumption that $H, J$ are cocommutative. It follows that $\zeta$ is continuous, since the action $(H) \otimes M \rightarrow M$ is. Moreover, $\zeta$ is a homeomorphism, since it has a continuous inverse given by

$$
\zeta^{-1}(m \otimes a \otimes p)=\sum S\left(a_{(1)}\right) m \otimes a_{(2)} \otimes p .
$$

Hence we may show for our purpose that

$$
\operatorname{Hom}_{\mathcal{D}}(L, \mathcal{Q}) \cong \operatorname{Hom}_{\mathrm{c}, J}(M \otimes(P), \mathcal{Q}) .
$$

There is an isomorphism $\check{\phi} \leftrightarrow \phi, \operatorname{Hom}_{H}(L, \mathcal{Q}) \cong \operatorname{Hom}(M \otimes P, \mathcal{Q})$, such that $\check{\phi}(m \otimes a \otimes p)=a \phi(m \otimes p)$. It is easy to see that $\check{\phi}$ is continuous if and only if $\phi$ is. Suppose $\check{\phi}$ is $J$-linear, that is,

$$
\sum \check{\phi}\left(\left(x_{(1)} \triangleleft a_{(1)}\right) m \otimes\left(x_{(2)} \triangleright a_{(2)}\right) \otimes\left(x_{(3)} \triangleleft a_{(3)}\right) p\right)=x \check{\phi}(m \otimes a \otimes p)
$$

for $x \in J, a \in H, m \in M, p \in P$. Suppose $a=1$. Then we have

$$
\sum \phi\left(x_{(1)} m \otimes x_{(2)} p\right)=x \phi(m \otimes p),
$$

which means that $\phi$ is $J$-linear.

Conversely, suppose $\phi$ is $J$-linear, so

$$
\sum \phi\left(\left(x_{(1)} \triangleleft a_{(1)}\right) m \otimes\left(x_{(2)} \triangleleft a_{(2)}\right) p\right)=(x \triangleleft a) \phi(m \otimes p)
$$

for $x \in J, a \in H, m \in M, p \in P$. Then we have

$$
\begin{aligned}
& \sum\left(x_{(1)} \triangleright a_{(1)}\right) \phi\left(\left(x_{(2)} \triangleleft a_{(2)}\right) m \otimes\left(x_{(3)} \triangleleft a_{(3)}\right) p\right) \\
& =\sum\left(x_{(1)} \triangleright a_{(1)}\right)\left(x_{(2)} \triangleleft a_{(2)}\right) \phi(m \otimes p)=x a \phi(m \otimes p) .
\end{aligned}
$$

This implies that $\check{\phi}$ is $J$-linear, since $H, J$ are cocommutative. Thus we are done.

Corollary 6.14. Let $P, Q$ be as above, and write $L=\operatorname{Hom}(F(P), G(Q))$. Suppose that a morphism $\phi: M \rightarrow N$ in $\mathcal{D}$ is strict [B1 Chap. III, Sect. 2, Definition 2] in the sense that the $H \bowtie J$-linear isomorphism $\bar{\phi}: M / \operatorname{Ker} \phi \stackrel{\sim}{\rightarrow} \operatorname{Im} \phi$ induced from $\phi$ is a homeomorphism. Then, if $\chi: M \rightarrow L$ is a morphism in $\mathcal{D}$ such that $\chi(\operatorname{Ker} \phi)=0$, there exists a morphism $\vartheta: N \rightarrow L$ in $\mathcal{D}$ such that $\chi=\vartheta \phi$. 
Proof. Since $\chi(\operatorname{Ker} \phi)=0$ and $\bar{\phi}$ is an isomorphism in $\mathcal{D}$, there is a morphism $\vartheta^{\prime}: \operatorname{Im} \phi \rightarrow L$ in $\mathcal{D}$ such that $\chi=\vartheta^{\prime} \bar{\phi} \pi$ with $\pi: M \rightarrow M / \operatorname{Ker} \phi$ the projection. It follows by Corollary 6.3 that the restriction map

$$
\operatorname{Hom}_{\mathrm{c}}(N, \operatorname{Hom}(P, Q)) \rightarrow \operatorname{Hom}_{\mathrm{c}}(\operatorname{Im} \phi, \operatorname{Hom}(P, Q))
$$

is a surjection. This implies by Proposition 6.13 that the restriction map

$$
\operatorname{Hom}_{\mathcal{D}}(N, L) \rightarrow \operatorname{Hom}_{\mathcal{D}}(\operatorname{Im} \phi, L)
$$

is a surjection. Now, choose $\vartheta$ in $\operatorname{Hom}_{\mathcal{D}}(N, L)$ that is mapped to $\vartheta^{\prime}$ through the last surjection.

To complete the proof of Theorem 4.11, let us return to the diagram (4.21). Since we see that $\varphi .: X .(\mathfrak{f}) \rightarrow X .(H)$ and $\psi^{*}: Y^{\cdot}(K) \rightarrow Y^{\cdot}\left(\mathfrak{g}^{*}\right)$ are both maps of complexes in the category $\mathcal{C}$, it follows from Proposition 6.112 ) that (4.21) is a commutative diagram in the category $\mathcal{D}$, with $\operatorname{Im} d^{1}, \operatorname{Im} \partial^{1}$ given the quotient topologies. Notice that each of $B^{i}, C^{i}(i=0,1)$ is a finite product of objects in the form $\operatorname{Hom}(F(P), G(Q))$, and hence that Corollary 6.14 can apply. Then the familiar argument for uniqueness of injective resolutions shows that $\alpha^{0}, \alpha^{1}, \alpha^{2}$, $0, \cdots$ give a homotopy equivalence between the deleted complexes in $\mathcal{D}$ (hence in $H \bowtie J$-Mod), as desired, if we prove, finally, the following lemma.

Lemma 6.15. The morphisms $d^{0}, \partial^{0}, e, \eta$ in (4.21) are all strict in the sense defined in Corollary 6.14.

Proof. Let $i: V^{\prime} \rightarrow V, j: W \rightarrow W^{\prime}$ be linear maps between (discrete) vector spaces. Let

$$
i^{\#}: \operatorname{Hom}(V, W) \rightarrow \operatorname{Hom}\left(V^{\prime}, W\right), \quad j_{\#}: \operatorname{Hom}(V, W) \rightarrow \operatorname{Hom}\left(V, W^{\prime}\right)
$$

denote the induced continuous linear maps between the topological vector spaces.

To show that $d^{0}, \partial^{0}$ are strict, it suffices to prove that the continuous linear map

$$
\left(i^{\#}, j_{\#}\right): \operatorname{Hom}(V, W) \rightarrow \operatorname{Hom}\left(V^{\prime}, W\right) \times \operatorname{Hom}\left(V, W^{\prime}\right)
$$

induced from $i^{\#}, j_{\#}$ is strict. Define $V_{0}^{\prime}=\operatorname{Ker} i, V_{1}=\operatorname{Im} i$. Then we may identify $i$ with the map

$$
V^{\prime}=V_{0}^{\prime} \oplus V_{1} \stackrel{0 \oplus 1}{\rightarrow} V_{0} \oplus V_{1}=V
$$

where $V_{0}$ is a complement of $V_{1}$ in $V$. Let $\left\{u_{\lambda}\right\},\left\{v_{\mu}\right\},\left\{w_{\gamma}\right\}$ be bases of $V_{0}, V_{1}, V_{0}^{\prime}$, respectively. Let $W_{\lambda}, W_{\mu}, W_{\gamma}$ be copies of $W$, and let $W_{\lambda}^{\prime}, W_{\mu}^{\prime}$ be copies of $W^{\prime}$. Then, $\left(i^{\#}, j_{\#}\right)$ is identified with the product

$$
\prod_{\lambda} W_{\lambda} \times \prod_{\mu} W_{\mu} \times \prod_{\gamma}\{0\} \rightarrow \prod_{\lambda} W_{\lambda}^{\prime} \times \prod_{\mu}\left(W_{\mu} \times W_{\mu}^{\prime}\right) \times \prod_{\gamma} W_{\gamma}
$$

of the linear maps

$$
j: W_{\lambda} \rightarrow W_{\lambda}^{\prime}, \quad(1, j): W_{\mu} \rightarrow W_{\mu} \times W_{\mu}^{\prime}, \quad 0:\{0\} \rightarrow W_{\gamma} .
$$

Since each of these maps is trivially strict, so is $\left(i^{\#}, j_{\#}\right)$.

If we suppose that $i$ is a surjection and $j$ is an injection, then it follows similarly that $i^{\#}, j_{\#}$ and hence $i^{\#} j_{\#}$ are strict, which yields that $e, \eta$ are strict. 


\section{ACKNOWLEDGEMENTS}

My first thanks go to Professor Hans-Jürgen Schneider, who allowed me to include in this paper his unpublished results (Theorems 5.2 and 5.3) with their proofs, gave me valuable suggestions in our delightful discussions, and informed me of interesting articles including, [Don, $[\mathrm{H}],[\mathrm{H} 1],[\mathrm{H} 2,[\mathrm{~K}],[\mathrm{L}]$. I am grateful to Professor Bodo Pareigis and Professor Mitsuhiro Takeuchi, who gave me helpful comments with heartful encouragement. Finally I want to thank Dr. Nicolás Andruskiewitsch and his students for their interest in this work and fruitful discussions.

\section{REFERENCES}

[A] N. Andruskiewitsch, Note on extensions of Hopf algebras (with an appendix by N. Andruskiewitsch and H.-J. Schneider), Can. J. Math. 48(1996), 3-42. MR 97c:16046

[BCM] R.J. Blattner, M. Cohen and S. Montgomery, Crossed products and inner actions of Hopf algebras, Trans. Amer. Math. Soc. 298(1986), 671-711. MR 87k:16012

[B] N. Bourbaki, "Lie Groups and Lie Algebras", Part I, Hermann, Paris, 1975. MR 89k:17001 (reprint)

[B1] N. Bourbaki, "General Topology", Part I, Hermann, Paris, 1966. MR 34:5044a

[CE] H, Cartan and S. Eilenberg, "Homological Algebra", Princeton Univ. Press, Princeton, 1956. MR 17:1040e

[Doi] Y. Doi, Algebras with total integrals, Comm. Algebra 13(1985), 2137-2159. MR 87c:16013

[Don] S. Donkin, On the Hopf algebra dual of an enveloping algebra, Math. Proc. Cambridge Phil. Soc. 91(1982), 215-224. MR 83h:16014

[D] V. G. Drinfeld, Quantum groups, Proc. ICM-86, Berkeley, 1987, 798-820. MR 89f:17017

[HS] P. Hilton and U. Stammbach, "A Course in Homological Algebra", Graduate Texts in Math., Vol.4, Springer-Verlag, Berlin, 1971. MR 49:10751

$[\mathrm{H}] \quad$ G. Hochschild, Algebraic Lie algebras and representative functions, Illinois J. Math. 3(1959), 499-523. MR 23:A3207

[H1] G. Hochschild, Algebraic Lie algebras and representative functions, supplements, Illinois J. Math. 4(1960), 609-618. MR 22:A3208

[H2] G. Hochschild, Algebraic groups and Hopf algebras, Illinois J. Math. 14(1970), 52-65. MR 41:1742

[Hf] I. Hofstetter, Extensions of Hopf algebras and their cohomological description, J. Algebra 164(1994), 264-298. MR 95e:16035

[Kac] G. I. Kac, Extensions of groups to ring groups, Math. USSR Sbornik 5(1968), 451-474. MR 37:4639

[Kas] C. Kassel, "Quantum Groups", Graduate Texts in Math., Vol.155, Springer-Verlag, New York, 1995. MR 96e:17041

[K] J. Koszul, Sur les modules de représentations des algèbres de Lie resolubles, Amer. Math. J. 76(1954), 535-554. MR 15:928e

[L] T. Levasseur, L'enveloppe injective du module trivial sur une algèbre de Lie resoluble, Bull. Sc. math., $2^{\mathrm{e}}$ série 110(1986), 49-61. MR 88c:17015

[Mj] S. Majid, "Foundations of Quantum Group Theory", Cambridge Univ. Press, Cambridge, 1995. MR 97g:17016

$[\mathrm{M}]$ A. Masuoka, Calculations of some groups of Hopf algebra extensions, J. Algebra 191(1997), 568-588; Corrigendum, J. Algebra 197(1997), 656. MR 98i:16042, MR 99f:16042

[MD] A. Masuoka and Y. Doi, Generalization of cleft comodule algebras, Comm. Algebra 20(1992), 3703-3721. MR 93j:16030

[Mi] W. Michaelis, Lie coalgebras, Adv. in Math. 38(1980), 1-54. MR 82g:17016

[Mo] S. Montgomery, "Hopf Algebras and Their Actions on Rings", CBMS Regional Conference Series in Math., Vol.82, Amer. Math. Soc., Providence, 1993. MR 94i:16019

[Mo1] S. Montgomery, Crossed products of Hopf algebras and enveloping algebras, in: Perspective in Ring Theory, Kluwer, 1988, 253-268. MR 91i:16056

[R] J. J. Rotman, "Introduction to Homological Algebra", Academic Press, New York, 1979. MR 80k:18001 
[S] W. M. Singer, Extension theory for connected Hopf algebras, J. Algebra 21(1972), 1-16. MR 47:8597

[Sw] M. E. Sweedler, Cohomology of algebras over Hopf algebras, Trans. Amer. Math. Soc. 127(1968), 205-239. MR 37:283

[T] M. Takeuchi, Matched pairs of groups and bismash products of Hopf algebras, Comm. Algebra 9(1981), 841-882. MR 83f:16013

[T1] M. Takeuchi, Topological coalgebras, J. Algebra 87(1985), 505-539. MR 87e:16028

[Wa] W. C. Waterhouse, "Introduction to Affine Group Schemes", Graduate Texts in Math., Vol.66, Springer-Verlag, New York, 1979. MR 82e:14003

[We] C. A. Weibel, "Introduction to Homological Algebra", Cambridge Univ. Press, Cambridge, 1994. MR 95f:18001

Mathematisches Institut der Universität MünChen, Theresienstr. 39, D-80333 MünChen, Germany

On leave of absence from: Department of Mathematics, Shimane University, Matsue, SHIMANE 690, JAPAN

Current address: Institute of Mathematics, University of Tsukuba, Tsukuba, Ibaraki 305-8571, Japan

E-mail address: akira@math.tsukuba.ac.jp 